\title{
Finding Four Independent Trees
}

\author{
Sean Curran* \\ School of Mathematics \\ Georgia Institute of Technology \\ Atlanta, Georgia 30332 \\ Orlando Lee ${ }^{\dagger}$ \\ Instituto de Computação \\ Universidade Estadual de Campinas \\ Avenida Albert Einstein, 1251 \\ Caixa Postal 6176 \\ 13083-971 Campinas - SP, Brazil \\ Xingxing $\mathrm{Yu}^{\ddagger}$ \\ School of Mathematics \\ Georgia Institute of Technology \\ Atlanta, Georgia 30332
}

\begin{abstract}
Motivated by a multi-tree approach to the design of reliable communication protocols, Itai and Rodeh gave a linear time algorithm for finding two independent spanning trees in a 2-connected graph. Cheriyan and Maheshwari gave an $O\left(|V|^{2}\right)$ algorithm for finding three independent spanning trees in a 3-connected graph. In this paper we present an $O\left(|V|^{3}\right)$ algorithm for finding four independent spanning trees in a 4-connected graph. We make use of chain decompositions of 4-connected graphs.
\end{abstract}

*Partially supported by NSF VIGRE grant

†Supported by CNPq (Proc: 200611/00-3) - Brazil

$\ddagger$ Partially supported by NSF grant DMS-0245530 and NSA grant MDA-904-03-1-0052 


\section{Introduction}

We consider simple graphs only. For a graph $G$, we use $V(G)$ and $E(G)$ to denote the vertex set and edge set of $G$, respectively.

For a tree $T$ and $x, y \in V(T)$, let $T[x, y]$ denote the unique path from $x$ to $y$ in $T$. A rooted tree is a tree with a specified vertex called the root of $T$. Let $G$ be a graph, let $r \in V(G)$, and let $T$ and $T^{\prime}$ be trees of $G$ rooted at $r$. We say that $T$ and $T^{\prime}$ are independent if for every $x \in V(T) \cap V\left(T^{\prime}\right)$, the paths $T[r, x], T^{\prime}[r, x]$ have no vertex in common except $r$ and $x$.

The study of independent spanning trees started with Itai and Rodeh [10], who proposed a multi-tree approach to reliability in distributed networks (see also [5]). They developed a linear time algorithm that, given any vertex $r$ in a 2-connected graph $G$, finds two independent spanning trees of $G$ rooted at $r$. Later, Cheryian and Maheshwari [3] proved that for any vertex $r$ in a 3connected graph $G$, there exist three independent spanning trees of $G$ rooted at $r$. Furthermore, they gave an $O\left(|V(G)|^{2}\right)$ algorithm for finding these trees.

Itai and Zehavi [11] also proved that every 3-connected graph contains three independent spanning trees (rooted at any vertex), and they conjectured that for any $k$-connected graph $G$ and for any $r \in V(G)$, there exist $k$ independent spanning trees of $G$ rooted at $r$. Huck [8] proved this conjecture for planar 4-connected graphs. Later, Miura, Nakano, Nishizeki and Takahashi [12] gave a linear time algorithm for finding four independent rooted spanning trees in a planar 4-connected graph.

Our main result is the following.

(1.1) Theorem. Let $G$ be a 4-connected graph and let $r \in V(G)$. Then there exist four independent spanning trees of $G$ rooted at $r$. Moreover, such trees can be found in $O\left(|V(G)|^{3}\right)$ time.

To provide motivation for our method, we first describe Itai and Rodeh's method for constructing two independent spanning trees rooted at a vertex $r$ in a 2-connected graph. Let $G$ be a 2-connected graph, and let $r$ and $t$ be two adjacent vertices of $G$. An $r$ - $t$ numbering is a function $g: V(G) \mapsto\{1, \ldots, n\}$ with $n \geq|V(G)|$ satisfying the following properties:

(i) $g(r)=1$ and $g(t)=n$, and

(ii) every vertex $v \in V(G)-\{r, t\}$ has a neighbor $u$ with $g(u)<g(v)$ and a neighbor $w$ with $g(w)>g(v)$.

An $r$ - $t$ numbering can be produced from an ear decomposition of $G$. From an $r$ - $t$ numbering $g$, Itai and Rodeh define two independent spanning trees $T_{1}$ and $T_{2}$ of $G$ rooted at $r$ as follows. For each vertex $v \in V(G)-\{r\}$, specify its parent in each tree. In tree $T_{1}$, for each $v \in V(G)-\{r\}$, the parent of $v$ is a neighbor $u$ for which $g(u)<g(v)$. In tree $T_{2}$, the parent of $t$ is $r$ and, for each $v \in V(G)-\{r, t\}$, the parent of $v$ is a neighbor $w$ for which $g(w)>g(u)$. It is not hard to show that $T_{1}$ and $T_{2}$ are independent spanning trees in $G$ rooted at $r$.

The idea for constructing four independent spanning trees in a 4-connected graph is inspired by the 2-connected case. The main difference is that we need to use two numberings instead of one. This idea can be roughly described as follows. Let $G$ be a 4-connected graph and let $r \in V(G)$. First, we compute a decomposition of $G$ into "planar chains", a generalization of ear decomposition, which we describe in Section 2. From this decomposition, we find two numberings $g$ and $f$. We then construct these trees from these numberings.

The main difficulty with this idea lies in the fact that it is not possible to number all vertices of $G$, because the "chains" in our decomposition need not be paths. Fortunately, the non-path part of the chains are planar, and we can compute four independent spanning trees in each one of these planar parts using the algorithm of Miura et al [12] mentioned above. These trees are then used to number every vertex in the planar parts that has neighbors outside its chain. Once these numberings are computed, we can construct four independent spanning trees. 
The rest of this paper is organized as follows. The remainder of this section is devoted to notation and terminology. In Section 2, we describe chain decomposition of a graph, and state the main decomposition result from [2] (also see [1]). In Section 3 we describe known results for the planar case and give some auxiliary lemmas. In Section 4 we give algorithms for constructing the required numberings. In Section 5 we describe an algorithm for constructing four independent spanning trees in a 4-connected graph, and we prove its correctness in Section 6 .

Throughout this paper, we use $A:=B$ to rename $B$ as $A$, or to define $A$ as $B$. We use the notation $x y$ (or $y x$ ) to represent an edge with ends $x$ and $y$. Let $G$ be graph. For any $S \subseteq V(G)$, let $G[S]$ denote the subgraph of $G$ with $V(G[S])=S$ and $E(G[S])$ consisting of the edges of $G$ with both ends in $S$; we say that $G[S]$ is the subgraph of $G$ induced by $S$. Let $G-S:=G[V(G)-S]$. A subgraph $H$ of $G$ is an induced subgraph of $G$ if $G[V(H)]=H$. We also say that $H$ is induced in $G$. For any $H \subseteq G$ and $S \subseteq V(G) \cup E(G), H+S$ denote the graph with vertex set $V(H) \cup(S \cap V(G))$ and edge set $E(H) \cup\{u v \in S \cap E(G):\{u, v\} \subseteq V(H) \cap(S \cap V(G))$.

A graph $G$ is $k$-connected, where $k$ is a positive integer, if $|V(G)| \geq k+1$ and, for any $S \subset V(G)$ with $|S| \leq k-1, G-S$ is connected. A subgraph $H$ of $G$ is non-separating in $G$ if $G-V(H)$ is connected.

Let $G$ be a graph. For $S \subseteq V(G)$, let $N_{G}(S):=\{x \in V(G)-S: x y \in E(G)$ for some $y \in S\}$. For a subgraph $H$ of $\bar{G}$, let $N_{G}(H):=N_{G}(V(H))$. When $S=\{x\}$, we let $N_{G}(x):=$ $N_{G}(\{x\})$. When there exists no ambiguity, we may simply use $N(S), N(H)$ and $N(x)$, instead of $N_{G}(S), N_{G}(H)$ and $N_{G}(x)$, respectively.

We describe a path in $G$ as a sequence $P=\left(v_{1}, v_{2}, \ldots, v_{k}\right)$ of distinct vertices of $G$ such that $v_{i} v_{i+1} \in E(G), 1 \leq i \leq k-1$. The vertices $v_{1}$ and $v_{k}$ are called the ends of the path $P$, and the vertices in $V(P)-\left\{v_{1}, v_{k}\right\}$ are called the internal vertices of $P$. For $1 \leq i \leq j \leq k$, let $P\left[v_{i}, v_{j}\right]:=\left(v_{i}, \ldots, v_{j}\right)$, and for $1 \leq i<j \leq k$, let $P\left(v_{i}, v_{j}\right):=P\left[v_{i+1}, v_{j-1}\right]$. For $A, B \subseteq V(G)$, we say that a path $P$ is an $A-B$ path if one end of $P$ is in $A$, the other end is in $B$, and no internal vertex of $P$ is in $A \cup B$. If $P$ is a path with ends $a$ and $b$, we say that $P$ is a path from $a$ to $b$, or $P$ is an $a-b$ path. Two paths $P$ and $Q$ are disjoint if $V(P) \cap V(Q)=\emptyset$. Two paths are internally disjoint if no internal vertex of one path is contained in the other. Given a path $P$ in $G$ and a set $S \subseteq V(G)$ (respectively, a subgraph $S$ of $G$ ), we say that $P$ is internally disjoint from $S$ if no internal vertex of $P$ is contained in $S$ (respectively, $V(S)$ ). We also describe a cycle in $G$ as a sequence $C=\left(v_{1}, v_{2}, \ldots, v_{k}, v_{1}\right)$ such that the vertices $v_{1}, \ldots, v_{k}$ are distinct, $v_{i} v_{i+1} \in E(G)$, for $1 \leq i \leq k-1$, and $v_{k} v_{1} \in E(G)$.

\section{Chain Decomposition}

In order to prove Theorem (1.1), we rely on the existence of a non-separating chain decomposition of a 4-connected graph, proved in [2] (also see [1]). Such a decomposition is similar to an ear decomposition. An ear decomposition of a graph $G$ is a sequence $\left(P_{0}, P_{1}, \ldots, P_{t}\right)$ such that (i) $P_{0}$ is a cycle in $G$, (ii) $P_{1}, \ldots, P_{t}$ are paths in $G$, (iii) $\bigcup_{i=0}^{t} P_{i}=G$, and (iv) for each $0 \leq i \leq t-1, G_{i}:=G\left[\bigcup_{j=0}^{i} V\left(P_{j}\right)\right]$ is 2-connected and $P_{i+1} \cap G_{i}$ consists of the ends of $P_{i+1}$. In a non-separating chain decomposition, the $P_{i}$ 's will be chains and cycle chains, which may be thought of as a generalization of paths and cycles.

(2.1) Definition. A connected graph $H$ is a chain if its blocks can be labeled as $B_{1}, \ldots, B_{k}$, where $k \geq 1$ is an integer, and its cut vertices can be labeled as $v_{1}, \ldots, v_{k-1}$ such that

(i) $V\left(B_{i}\right) \cap V\left(B_{i+1}\right)=\left\{v_{i}\right\}$ for $1 \leq i \leq k-1$, and

(ii) $V\left(B_{i}\right) \cap V\left(B_{j}\right)=\emptyset$ if $|i-j| \geq 2$ and $1 \leq i, j \leq k$.

We let $H:=B_{1} v_{1} B_{2} v_{2} \ldots v_{k-1} B_{k}$ denote this situation. If $k \geq 2$, let $v_{0} \in V\left(B_{1}\right)-\left\{v_{1}\right\}$ and $v_{k} \in V\left(B_{k}\right)-\left\{v_{k-1}\right\}$, or, if $k=1$, let $v_{0}, v_{k} \in V\left(B_{1}\right)$ with $v_{0} \neq v_{k}$, then we say that $H$ is a $v_{0}-v_{k}$ chain, and we denote this by $H:=v_{0} B_{1} v_{1} \ldots v_{k-1} B_{k} v_{k}$. We usually fix $v_{0}$ and $v_{k}$, and we refer to them as the ends of $H_{i}$. See Figure 1 for an example with $k=5$. 


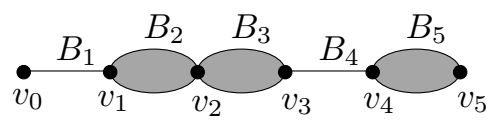

Figure 1: Example of a chain.

(2.2) Definition. A connected graph $H$ is a cyclic chain if for some integer $k \geq 2$, there exist subgraphs $B_{1}, \ldots, B_{k}$ of $H$ and vertices $v_{1}, \ldots, v_{k}$ of $H$ such that

(i) for $1 \leq i \leq k, B_{i}$ is 2-connected or $B_{i}$ is induced by an edge of $H$,

(ii) $V(H)=\bigcup_{i=1}^{k} V\left(B_{i}\right)$ and $E(H)=\bigcup_{i=1}^{k} E\left(B_{i}\right)$,

(iii) if $k=2$, then $V\left(B_{1}\right) \cap V\left(B_{2}\right)=\left\{v_{1}, v_{2}\right\}$ and $E\left(B_{1}\right) \cap E\left(B_{2}\right)=\emptyset$, and

(iv) if $k \geq 3$, then $V\left(B_{i}\right) \cap V\left(B_{i+1}\right)=\left\{v_{i}\right\}$ for $1 \leq i \leq k$ where $B_{k+1}:=B_{1}$, and $V\left(B_{i}\right) \cap V\left(B_{j}\right)=$ $\emptyset$ for $1 \leq i<i+2 \leq j \leq k$ and $(i, j) \neq(1, k)$.

For notational convenience, we usually fix one of the vertices $v_{1}, \ldots, v_{k}$ as the root of $H$, say $v_{k}$, and write $H:=v_{0} B_{1} v_{1} \ldots v_{k-1} B_{k} v_{k}$ to indicate that $H$ is a cyclic chain rooted at $v_{0}\left(=v_{k}\right)$. Each subgraph $B_{i}$ is called a piece of $H$. We sometimes write $I(H):=V(H)$. See Figure 2 for an example with $k=6$.

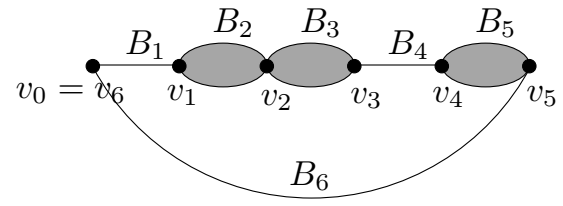

Figure 2: Example of a cyclic chain.

In the chain decompositions we will work with, the blocks and pieces have a planar structure. Let $G$ be a graph with distinct vertices $a, b, c$ and $d$. We say that the quintuple $(G, a, b, c, d)$ is planar if $G$ can be drawn in a closed disc in the plane with no pair of edges crossing such that $a, b, c, d$ occur in cyclic order on the boundary of the disc. For a graph $G$ and $x, y \in V(G)$, we use $G-x y$ to denote the graph with vertex set $V(G)$ and edge set $E(G)-\{x y\}$ (note that $x y$ need not be an edge of $G$ ).

(2.3) Definition. Let $G$ be a graph and let $H:=v_{0} B_{1} v_{1} \ldots v_{k-1} B_{k} v_{k}$ be a chain (respectively, cyclic chain). If $H$ is an induced subgraph of $G$, then we say that $H$ is a chain in $G$ (respectively, cyclic chain in $G)$. We say that $H$ is planar in $G$ if, for each $1 \leq i \leq k$ with $\left|V\left(B_{i}\right)\right| \geq 3$ (or equivalently, $B_{i}$ is 2-connected), there exist distinct vertices $x_{i}, y_{i} \in V(G)-V(H)$ such that $\left(G\left[V\left(B_{i}\right) \cup\left\{x_{i}, y_{i}\right\}\right]-x_{i} y_{i}, x_{i}, v_{i-1}, y_{i}, v_{i}\right)$ is planar, and $B_{i}-\left\{v_{i-1}, v_{i}\right\}$ is a component of $G-\left\{x_{i}, y_{i}, v_{i-1}, v_{i}\right\}$. We also say that $H$ is a planar $v_{0}-v_{k}$ chain. See Figure 3 for two drawings of an example with $k=5$. The dashed edges may or may not exist, but they are not part of $H$.

We can now describe the chains in non-separating chain decompositions. See Figure 4 for illustrations.

(2.4) Definition. Let $G$ be a graph, let $F$ be a subgraph of $G$, and let $r \in V(F)$. Let $H$ be a planar $x-y$ chain in $G$ such that $V(H)-\{x, y\} \subseteq V(G)-V(F)$. We say that

(a) $H$ is an up F-chain if $\{x, y\} \subseteq V(F)$ and $N_{G}(H-\{x, y\}) \subseteq(V(G)-V(F-r)) \cup\{x, y\}$, 

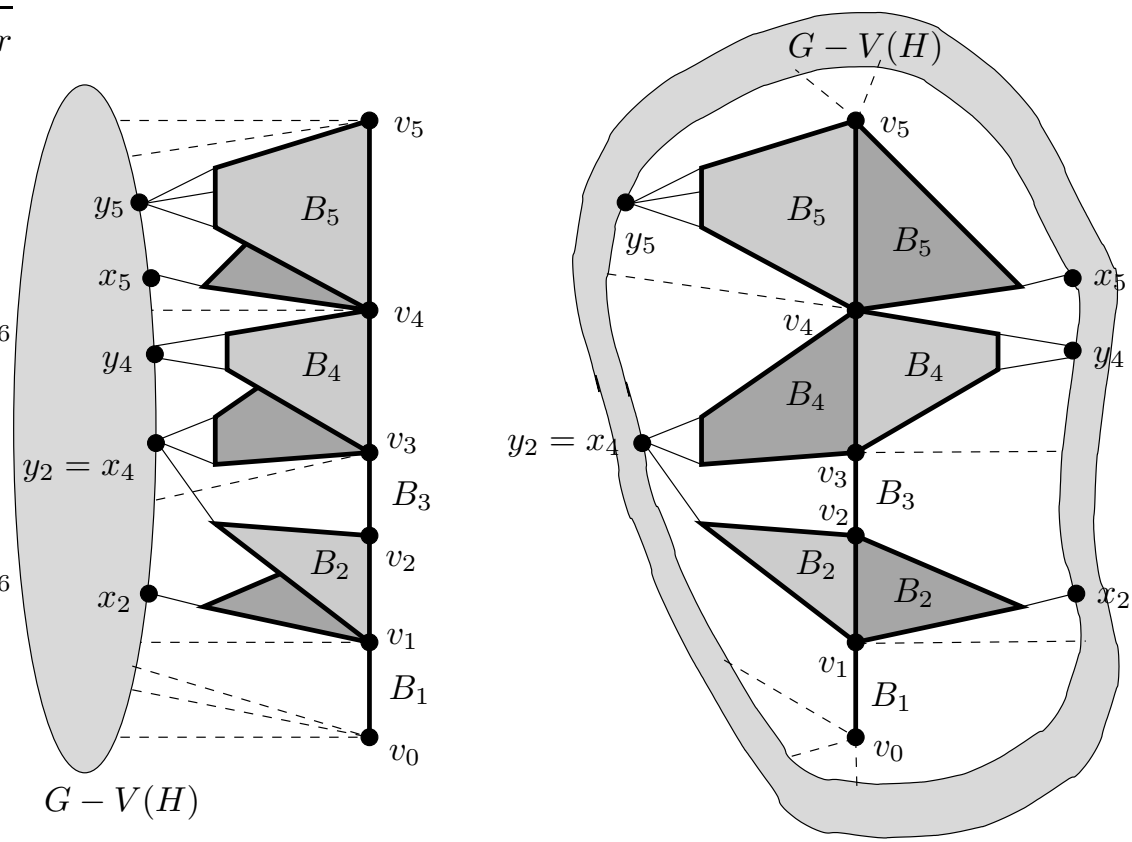

Figure 3: A planar chain $H:=v_{0} B_{1} v_{1} B_{2} v_{2} B_{3} v_{3} B_{4} v_{4} B_{5} v_{5}$ in a graph $G$.

(b) $H$ is a down $F$-chain if $\{x, y\} \subseteq V(G)-V(F-r)$ and $N_{G}(H-\{x, y\}) \subseteq V(F-r) \cup\{x, y\}$, and

(c) $H$ is an elementary $F$-chain if $\{x, y\} \subseteq V(F)$ and $H$ is an $x$-y path of length two.

In any of the three cases above we say that $H$ is a planar $x-y F$-chain in $G$ (or simply, a planar $F$-chain). Let $I(H):=V(H)-\{x, y\}$.

(2.5) Definition. Let $G$ be a graph, let $F$ be a subgraph of $G$, and let $r \in V(F)$. Suppose that $\left\{v_{1}, v_{2}, v_{3}\right\} \subseteq V(G)-V(F)$ induces a triangle $T$ in $G$ and, for each $1 \leq i \leq 3, v_{i}$ has exactly one neighbor $x_{i}$ in $V(F-r)$ and exactly one neighbor $y_{i}$ in $V(G)-\left(V(F) \cup V(T)\right.$ ) (thus, each $v_{i}$ has degree four in $G$ ). Moreover, assume that $x_{1}, x_{2}, x_{3}$ are distinct and $y_{1}, y_{2}, y_{3}$ are distinct. Then we say that $H:=T+\left\{x_{1}, x_{2}, x_{3}, v_{1} x_{1}, v_{2} x_{2}, v_{3} x_{3}\right\}$ is a triangle $F$-chain in $G$. We let $I(H):=\left\{v_{1}, v_{2}, v_{3}\right\}$.

Note those definitions depend on the choice of $r$ and $F$, but in spite of this, whenever we use these concepts in this paper, it should be clear which pair $r, F$ we refer to.

(2.6) Definition. Let $G$ be a graph, let $F$ be a subgraph of $G$, and let $r \in V(F)$. By a good $F$-chain in $G$, we mean an up $F$-chain, or a down $F$-chain, or an elementary $F$-chain, or a triangle $F$-chain.

We are now ready to describe a chain decomposition, which is similar to an ear decomposition.

(2.7) Definition. Let $G$ be a graph, let $r \in V(G)$, and let $H_{1}, \ldots, H_{t}$ be chains in $G$, where $t \geq 2$. We say that $\left(H_{1}, \ldots, H_{t}\right)$ is a non-separating chain decomposition of $G$ rooted at $r$ if the following conditions hold:

(i) $H_{1}$ is a planar cyclic chain in $G$ rooted at $r$,

(ii) for each $i=2, \ldots, t-1, H_{i}$ is a good $G\left[\bigcup_{j=1}^{i-1} I\left(H_{j}\right)\right]$-chain in $G$, 


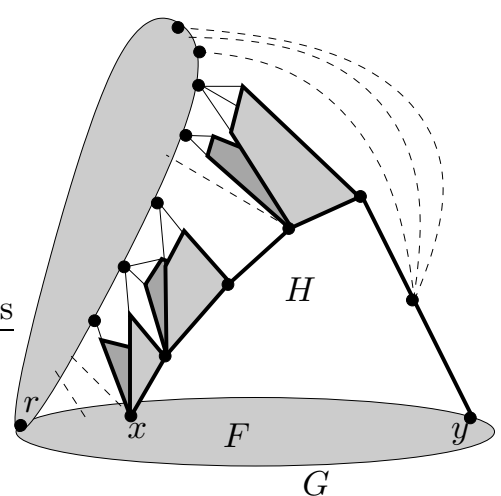

(a)

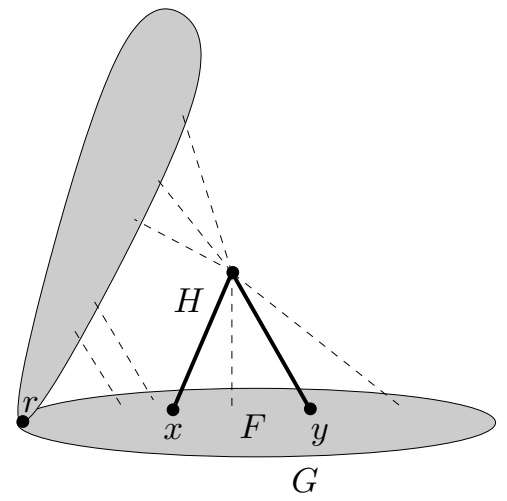

(c)

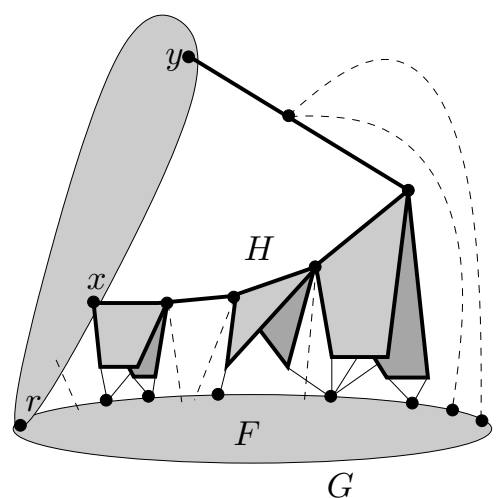

(b)

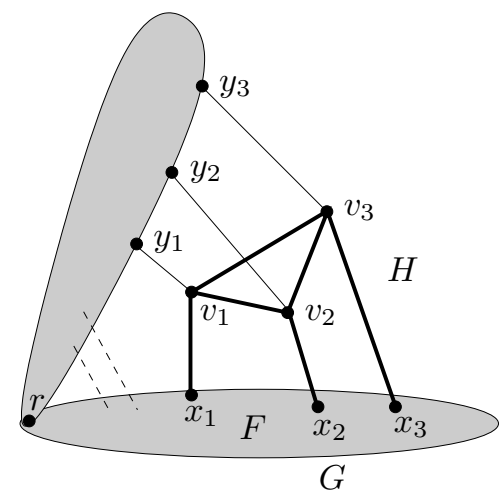

(d)

Figure 4: (a) An up $F$-chain, (b) a down $F$-chain, (c) an elementary $F$-chain, and (d) a triangle $F$-chain. The dashed edges need not exist.

(iii) $H_{t}:=G-\left(\bigcup_{j=1}^{t-1} I\left(H_{j}\right)-\{r\}\right)$ is a planar cyclic chain in $G$ rooted at $r$, and

(iv) for each $i=1, \ldots, t-1$, both $G\left[\bigcup_{j=1}^{i} I\left(H_{j}\right)\right]$ and $G-\left(\left(\bigcup_{j=1}^{i} I\left(H_{j}\right)\right)-\{r\}\right)$ are 2-connected.

The chains $H_{2}, \ldots, H_{t-1}$ are called internal chains of the non-separating chain decomposition. If $r a$ is a piece of $H_{1}$, then we say that $H_{1}, \ldots, H_{t}$ is a non-separating chain decomposition of $G$ starting at ra.

The following result is proved in [2] (also see [1]).

(2.8) Theorem. Let $G$ be a 4-connected graph, let $r \in V(G)$, and let $r a \in E(G)$. Then $G$ has a non-separating chain decomposition rooted at $r$ and starting at $r a$, and such a decomposition can be found in $O\left(|V(G)|^{2}|E(G)|\right)$ time.

The basic idea for constructing four independent spanning trees (rooted at $r$ ) can be described as follows. By $(2.8), G$ has a non-separating chain decomposition $\left(H_{1}, \ldots, H_{t}\right)$ rooted at $r$. For $1 \leq i \leq t$, let $G_{i}:=G\left[\bigcup_{j=1}^{i} I\left(H_{j}\right)\right]$. We compute two numberings $g, f$ defined on $V(G)$ which resemble $r$ - $t$ numberings. From $g$ we compute two independent spanning trees $T_{1}, T_{2}$ such that for each $i=1, \ldots, t$, the restriction of $T_{1}$ and $T_{2}$ to $G_{i}$ are independent spanning trees in $G_{i}$ rooted at $r$. Similarly, from $f$ we compute two spanning trees $T_{3}, T_{4}$ such that for each $i=1, \ldots, t$, the restriction of $T_{3}$ and $T_{4}$ to $G-\left(V\left(G_{i}-r\right)\right)$ are independent spanning trees rooted at $r$. 


\section{Planar graphs}

Let $G$ be a 4-connected graph and let $r \in V(G)$. To use a non-separating chain decomposition of $G$ for constructing four independent spanning trees rooted at $r$, we must be able to find four independent spanning trees in the planar blocks and pieces. Unlike the original problem, these trees are not rooted at the same vertex, but they are rooted at four distinct vertices. Before we describe this result, we introduce some definitions.

(3.1) Definition. Let $T$ and $T^{\prime}$ be two trees in a graph $G$ with roots $r$ and $r^{\prime}$, respectively. We say that $T$ and $T^{\prime}$ are independent if, for each $x \in V(T) \cap V\left(T^{\prime}\right)$, the paths $T[r, x]$ and $T^{\prime}\left[r^{\prime}, x\right]$ have no vertex in common except $x$ (and $r$, if $r=r^{\prime}$ ).

Let $G$ be a graph and let $S:=\left\{t_{1}, \ldots, t_{4}\right\}$ be a set of vertices of $G$. A 4 -tuple $\mathcal{T}:=\left\{T_{1}, \ldots, T_{4}\right\}$ is an $S$-system of $G$ if, for $1 \leq i \leq 4, T_{i}$ is a tree of $G$ rooted at $t_{i}, V\left(T_{i}\right) \subseteq V(G)-\left(S-\left\{t_{i}\right\}\right)$, and $t_{i} \in V\left(T_{i}\right)$. An $S$-system $\mathcal{T}:=\left\{T_{1}, \ldots, T_{4}\right\}$ is independent if the trees in the system are pairwise independent, and an $S$-system $\mathcal{T}$ is spanning if $V\left(T_{i}\right)=V(G)-\left(S-\left\{t_{i}\right\}\right)$ for $1 \leq i \leq 4$. See Figure 5 for an example, where the darkened edges are in the trees.
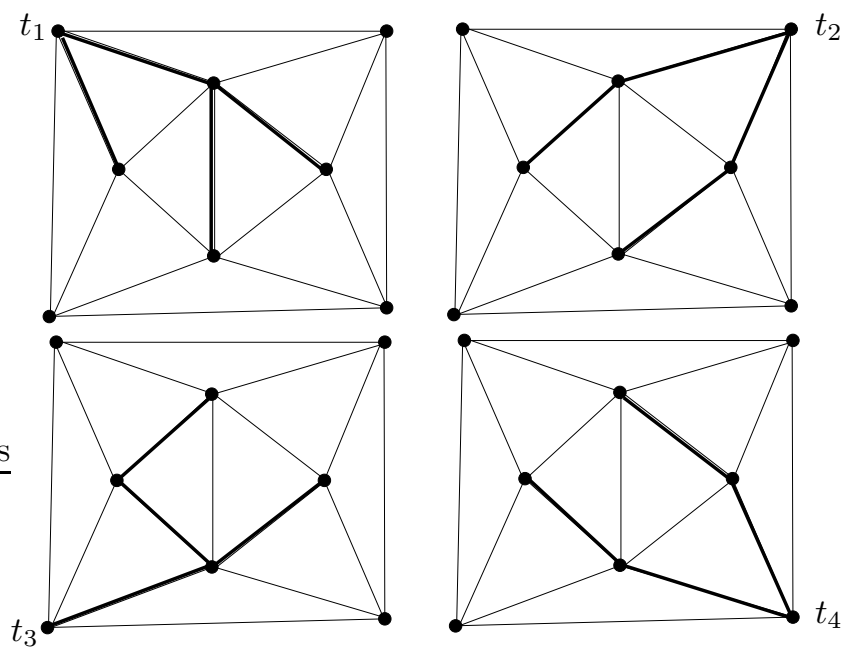

Figure 5: Four independent trees in a plane graph forming an independent spanning system.

Let $G$ be a graph, let $S \subseteq V(G)$, and let $k$ be a positive integer. We say that $G$ is $(k, S)$ connected if $|V(G)| \geq|S|+1, G$ is connected, and for any $T \subset V(G)$ with $|T| \leq k-1$, every component of $G-T$ contains an element of $S$.

(3.2) Theorem. Let $(G, a, b, c, d)$ be a planar graph and suppose that $G$ is $(4,\{a, b, c, d\})$ connected. Then there exists an independent spanning $\{a, b, c, d\}$-system of $G$. Moreover, one can find such a system in linear time.

The existence of an independent system in Theorem (3.2) was proved by Huck [8]. Huck's proof is not based on a decomposition of a planar graph, but through a careful analysis of his proof, one can extract an $O\left(|V(G)|^{3}\right)$ algorithm. Miura et al [12] gave a linear algorithm for finding such a system based on a decomposition of 4-connected planar graphs. In fact, the decomposition they obtained can be viewed as a special case of a non-separating-chain decomposition.

Before we proceed, let us recall that the problem of finding an embedding of a planar graph can be solved in linear time $[6,7]$. Moreover, the following problem can be solved in linear time: find a drawing of a planar quintuple $(G, a, b, c, d)$ in a closed disc in the plane with no pair of edges crossing such that $a, b, c, d$ occur in cyclic order on the boundary of the disc. We make no further mention of this fact, but it is implicitly used throughout this section. 
In what follows we will use Theorem (3.2) to prove some results concerning "orderings" of certain vertices of a planar graph $(G, a, b, c, d)$. These results correspond to Lemmas (3.4), (3.5), (3.6) and (3.7). They will be used in the next section to compute two numberings of subsets of $V(G)$.

(3.3) Definition. Let $(G, a, b, c, d)$ be a planar graph, and let $\left\{T_{a}, T_{b}, T_{c}, T_{d}\right\}$ be an independent spanning $\{a, b, c, d\}$-system of $G$, where $T_{v}$ is rooted at $v$ for each $v \in\{a, b, c, d\}$. Let $U \subseteq$ $\left(N_{G}(b) \cup N_{G}(d)\right)-\{a, c\}$. We say that a permutation $u_{1}, \ldots, u_{p}$ of the elements of $U$ is a $\left(T_{a}, T_{c}\right)$ ordering of $U$ if, for $i, j \in\{1, \ldots, p\}$ with $i<j, T_{a}\left[a, u_{i}\right]$ and $T_{c}\left[c, u_{j}\right]$ are (vertex) disjoint. We also say that $u_{1}, \ldots, u_{p}$ is $\left(T_{a}, T_{c}\right)$-ordered.

Our first lemma concerns $\left(T_{a}, T_{c}\right)$-orderings restricted to elements in $N_{G}(b)-\{a, c\}$. In this case, this ordering corresponds to a total order.

(3.4) Lemma. Let $(G, a, b, c, d)$ be a planar graph, and let $\left\{T_{a}, T_{b}, T_{c}, T_{d}\right\}$ be an independent spanning $\{a, b, c, d\}$-system of $G$, where $T_{v}$ is rooted at $v$ for each $v \in\{a, b, c, d\}$. Then there exists a unique $\left(T_{a}, T_{c}\right)$-ordering $u_{1}, \ldots, u_{p}$ of $N_{G}(b)-\{a, c\}$. Moreover, such an ordering can be found in $O(|V(G)|)$ time.

Proof. Let $G^{\prime}:=G-\{a b, b c\}$. If $b$ has exactly one neighbor in $G^{\prime}$, then the result follows immediately. So assume $b$ has at least two neighbors in $G^{\prime}$. Take an embedding of $G^{\prime}$ in a closed disc such that $a, b, c, d$ occur in clockwise order on the boundary of the disc. (Such an embedding for $G$ can be computed in linear time.) Let $u_{1}, \ldots, u_{p}(p \geq 2)$ denote the neighbors of $b$ in $G^{\prime}$ in that cyclic order around bsuch that $a, u_{1}, b, u_{p}, c, d$ occur in clockwise order on the boundary of the disc (see Figure 6). Since $T_{a}, T_{c}$ are independent, we have that for each $i \in\{1, \ldots, p\}, T_{a}\left[a, u_{i}\right]$ and $T_{c}\left[c, u_{i}\right]$ are internally disjoint. Then by planarity one can see that, for every $i, j \in\{1, \ldots, p\}$ with $i \neq j, T_{a}\left[a, u_{i}\right]$ and $T_{c}\left[c, u_{j}\right]$ are disjoint if, and only if, $i<j$. Thus, $u_{1}, \ldots, u_{p}$ is the unique $\left(T_{a}, T_{c}\right)$-ordering of $N_{G}(b)-\{a, c\}$. Clearly, such an ordering can be computed in $O(|V(G)|)$ time.

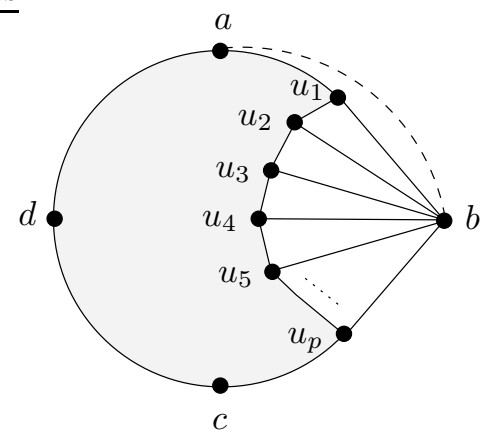

Figure 6: $u_{1}, \ldots, u_{p}$ is the unique $\left(T_{a}, T_{c}\right)$-ordering of $N_{G}(b)-\{a, c\}$.

In the next lemma we show that it is possible to extend a $\left(T_{a}, T_{c}\right)$-ordering of $N_{G}(b)-\{a, c\}$ and a $\left(T_{a}, T_{c}\right)$-ordering of $N_{G}(d)-\{a, c\}$ to a $\left(T_{a}, T_{c}\right)$-ordering of $\left(N_{G}(b) \cup N_{G}(d)\right)-\{a, c\}$.

(3.5) Lemma. Let $(G, a, b, c, d)$ be a planar graph, and let $\left\{T_{a}, T_{b}, T_{c}, T_{d}\right\}$ be an independent spanning $\{a, b, c, d\}$-system of $G$ where $T_{v}$ is rooted at $v$ for each $v \in\{a, b, c, d\}$. Then there exists a $\left(T_{a}, T_{c}\right)$-ordering of $\left(N_{G}(b) \cup N_{G}(d)\right)-\{a, c\}$. Moreover, such an ordering can be found in $O\left(|V(G)|^{2}\right)$ time.

Proof. Take an embedding of $G$ in a closed disc such that $a, b, c, d$ occur in clockwise order on the boundary of the disc. Consider the following relation. For $u, v \in\left(N_{G}(b) \cup N_{G}(d)\right)-\{a, c\}$, we say that $u \prec v$ if one of the following holds: 
(i) $u \in N_{G}(b)$, and $T_{a}[a, u] \cap T_{c}[c, v]=\emptyset$, or

(ii) $u \in N_{G}(d), T_{a}[a, u] \cap T_{c}[c, v]=\emptyset$, and $T_{a}[a, v] \cap T_{c}[c, u] \neq \emptyset$.

See Figure 7 for an illustration of conditions (i) and (ii). The bold lines denote the paths in $T_{a}$ and the dashed lines denote the paths in $T_{c}$. Next we show that $\prec$ defines a total order on $\left(N_{G}(b) \cup N_{G}(d)\right)-\{a, c\}$.

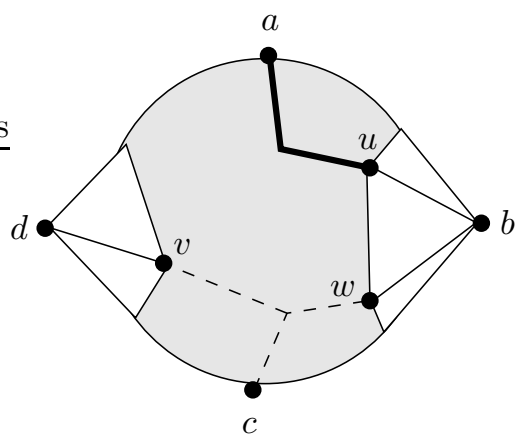

Condition (i)

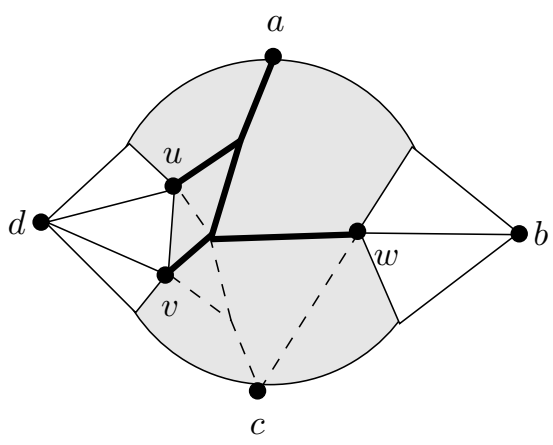

Condition (ii)

Figure 7: $u \prec v$ and $u \prec w$.

First, we show that for any distinct $x, y \in\left(N_{G}(b) \cup N_{G}(d)\right)-\{a, c\}$, either $x \prec y$, or $y \prec x$, but not both. If $x, y \in N_{G}(b)$ or $x, y \in N_{G}(d)$, then by planarity, either $T_{a}[a, x] \cap T_{c}[c, y]=\emptyset$ and $T_{a}[a, y] \cap T_{c}[c, x] \neq \emptyset$, or $T_{a}[a, x] \cap T_{c}[c, y] \neq \emptyset$ and $T_{a}[a, y] \cap T_{c}[c, x]=\emptyset$. So by (i) or (ii), either $x \prec y$, or $y \prec x$, but not both. Thus, we may assume that $x \in N_{G}(b)$ and $y \in N_{G}(d)$. If $T_{a}[a, x] \cap T_{c}[c, y]=\emptyset$, then $x, y$ satisfy (i) (as $u, v$ ) but not (ii) (as $v, u$ ), and we have $x \prec y$ and $y \nprec x$. So assume $T_{a}[a, x] \cap T_{c}[c, y] \neq \emptyset$. Then $x, y$ does not satisfy (i) (as $u, v$ ), and hence, $x \nprec y$. Since $T_{a}$ and $T_{c}$ are independent, $T_{a}[a, y]$ and $T_{c}[c, y]$ are internally disjoint, and $T_{a}[a, x]$ and $T_{c}[c, x]$ are internally disjoint. By planarity, $T_{a}[a, y] \cap T_{c}[c, x]=\emptyset$. Therefore, $y \prec x$.

It remains to show that $\prec$ is transitive. Let $x, y, z \in\left(N_{G}(b) \cup N_{G}(d)\right)-\{a, c\}$ and assume that $x \prec y$ and $y \prec z$. We will show that $x \prec z$. We have eight cases by considering which of $x, y, z$ are in $N_{G}(b)$.

(1) $x, y, z \in N_{G}(b)$. Since $x \prec y$ and $y \prec z$, it follows from (i) that $T_{a}[a, x] \cap T_{c}[c, y]=\emptyset$ and $T_{a}[a, y] \cap T_{c}[c, z]=\emptyset$. So by planarity, $T_{a}[a, x] \cap T_{c}[c, z]=\emptyset$, and by (i), $x \prec z$.

(2) $x, y, z \in N_{G}(d)$. Since $x \prec y$ and $y \prec z$, it follows from (ii) that $T_{a}[a, x] \cap T_{c}[c, y]=\emptyset$, $T_{a}[a, y] \cap T_{c}[c, x] \neq \emptyset, T_{a}[a, y] \cap T_{c}[c, z]=\emptyset$, and $T_{a}[a, z] \cap T_{c}[c, y] \neq \emptyset$. So by planarity, $T_{a}[a, x] \cap T_{c}[c, z]=\emptyset$ and $T_{a}[a, z] \cap T_{c}[c, x] \neq \emptyset$. By (ii), $x \prec z$.

(3) $y, z \in N_{G}(b)$ and $x \in N_{G}(d)$. Since $T_{a}$ and $T_{c}$ are independent, $P:=T_{a}[a, y] \cup T_{c}[c, y]$ is an $a-c$ path in $G-\{b, d\}$. Note that $P$ divides the disc into two closed regions, say $B$ and $D$, with $b$ in $B$ and $d$ in $D$. Since $x \prec y$ and $x \in N_{G}(d)$, it follows from (ii) that $T_{a}[a, x] \cap T_{c}[c, y]=\emptyset$ and $T_{a}[a, y] \cap T_{c}[c, x] \neq \emptyset$. Since $y \prec z$ and $y \in N_{G}(b)$, it follows from (i) that $T_{a}[a, y] \cap T_{c}[c, z]=\emptyset$. So $T_{c}[c, z]$ lies in $B$ and $T_{a}[a, x]$ lies in $D$. Therefore, by planarity, $T_{a}[a, x] \cap T_{c}[c, z]=\emptyset$. Since $T_{a}[a, y] \cap T_{c}[c, x] \neq \emptyset$, it follows by planarity that $T_{a}[a, z] \cap T_{c}[c, x] \neq \emptyset$. Therefore, $x \prec z$.

(4) $y, z \in N_{G}(d)$ and $x \in N_{G}(b)$. Since $T_{a}$ and $T_{c}$ are independent, $P:=T_{a}[a, y] \cup T_{c}[c, y]$ is an $a-c$ path in $G-\{b, d\}$, and $P$ divides the disc into two closed regions $B$ and $D$, with $b$ in $B$ and $d$ in $D$. Since $x \prec y$ and $x \in N_{G}(b)$, it follows from (i) that $T_{a}[a, x] \cap T_{c}[c, y]=\emptyset$, and since $y \prec z$ and $y \in N_{G}(d)$, it follows from (ii) that $T_{a}[a, y] \cap T_{c}[c, z]=\emptyset$. So $T_{c}[c, z]$ lies in $D$ and $T_{a}[a, x]$ lies in $B$. By planarity, $T_{a}[a, x] \cap T_{c}[c, z]=\emptyset$, and hence by (i), $x \prec z$. 
(5) $x, y \in N_{G}(d)$ and $z \in N_{G}(b)$. Since $T_{a}$ and $T_{c}$ are independent, $P:=T_{a}[a, y] \cup T_{c}[c, y]$ is an $a-c$ path in $G-\{b, d\}$, and $P$ divides the disc into two closed regions $B$ and $D$, with $b$ in $B$ and $d$ in $D$. Since $x \prec y$ and $x \in N_{G}(d)$, it follows from (ii) that $T_{a}[a, x] \cap T_{c}[c, y]=\emptyset$, and since $y \prec z$ and $y \in N_{G}(d)$, it follows from (ii) that $T_{a}[a, y] \cap T_{c}[c, z]=\emptyset$. Thus, $T_{a}[a, x]$ lies in $D$ and $T_{c}[c, z]$ lies in $B$. By planarity, $T_{a}[a, x] \cap T_{c}[c, z]=\emptyset$. Moreover, since $y \prec z$ and $y \in N_{G}(d)$, it follows from (ii) that $T_{a}[a, z] \cap T_{c}[c, y] \neq \emptyset$. By planarity, $T_{a}[a, z] \cap T_{c}[c, x] \neq \emptyset$. Therefore by (ii), $x \prec z$.

(6) $x, y \in N_{G}(b)$ and $z \in N_{G}(d)$. Since $T_{a}$ and $T_{c}$ are independent, $P:=T_{a}[a, y] \cup T_{c}[c, y]$ is an $a-c$ path in $G-\{b, d\}$, and $P$ divides the disc into two closed regions $B$ and $D$, with $b$ in $B$ and $d$ in $D$. Since $x \prec y$ and $x \in N_{G}(b)$, it follows from (i) that $T_{a}[a, x] \cap T_{c}[c, y]=\emptyset$, and since $y \prec z$ and $y \in N_{G}(b)$, it follows from (i) that $T_{a}[a, y] \cap T_{c}[c, z]=\emptyset$. So $T_{a}[a, x]$ lies in $B$ and $T_{c}[c, z]$ lies in $D$. By planarity $T_{a}[a, x] \cap T_{c}[c, z]=\emptyset$. Therefore by (i), $x \prec z$.

(7) $x, z \in N_{G}(b)$ and $y \in N_{G}(d)$. We have shown that either $x \prec z$ or $z \prec x$. Suppose for a contradiction that $z \prec x$. Then by (i), $T_{a}[a, z] \cap T_{c}[c, x]=\emptyset$, and by planarity, $T_{a}[a, x] \cap$ $T_{c}[c, z] \neq \emptyset$. Since $y \prec z$ and $y \in N_{G}(d), T_{a}[a, z] \cap T_{c}[c, y] \neq \emptyset$. But then, by planarity, $T_{a}[a, x] \cap T_{c}[c, y] \neq \emptyset$, which is a contradiction to (i) since $x \prec y$ and $x \in N_{G}(b)$. Therefore, $x \prec z$.

(8) $x, z \in N_{G}(d)$ and $y \in N_{G}(b)$. We have shown that either $x \prec z$ or $z \prec x$. Suppose for a contradiction that $z \prec x$. Then by (ii), $T_{a}[a, z] \cap T_{c}[c, x]=\emptyset$ and $T_{a}[a, x] \cap T_{c}[c, z] \neq \emptyset$. Since $x \prec y$ and $x \in N_{G}(d), T_{a}[a, y] \cap T_{c}[c, x] \neq \emptyset$. But then, by planarity, $T_{a}[a, y] \cap T_{c}[c, z] \neq \emptyset$, which is a contradiction to (i) since $y \prec z$ and $y \in N_{G}(b)$. Therefore, $x \prec z$.

Thus, $\prec$ defines a total order on $\left(N_{G}(b) \cup N_{G}(d)\right)-\{a, c\}$. Hence, the required $\left(T_{a}, T_{c}\right)$-ordering exists.

Furthermore, this ordering can be found as follows. Let $b_{1}, \ldots, b_{p}$ be the $\left(T_{a}, T_{c}\right)$-ordering of $N_{G}(b)-\{a, c\}$, and let $d_{1}, \ldots, d_{q}$ be the $\left(T_{a}, T_{c}\right)$-ordering of $N_{G}(d)-\{a, c\}$. Both exist by Lemma (3.4). Theses sequences are ordered under $\prec$. We can decide in $O(|V(G)|)$ time whether $b_{i} \prec d_{j}$ or $d_{j} \prec b_{i}$ (by checking which of (i) or (ii) holds) for any pair $b_{i}, d_{j}, 1 \leq i \leq p, 1 \leq j \leq q$. Thus, using the so called "merge technique" in [4], we can merge the two sequences to obtain a sequence ordered under $\prec$ in $O\left(|V(G)|^{2}\right)$ time.

The last two lemmas of this section will also be needed in Section 5 . Figure 8 illustrates Lemma (3.6), and Figure 9 illustrates Lemma (3.7).

(3.6) Lemma. Let $(G, a, b, c, d)$ be a planar graph, and let $\left\{T_{a}, T_{b}, T_{c}, T_{d}\right\}$ be an independent spanning $\{a, b, c, d\}$-system of $G$, where $T_{v}$ is rooted at $v$ for each $v \in\{a, b, c, d\}$. Assume that $b$ has at least two neighbors in $V(G)-\{a, c\}$. Then for any $\left(T_{a}, T_{c}\right)$-ordered pair $x, y \in$ $N_{G}(b)-\{a, c\}, T_{a}[a, x] \cap T_{d}[d, y]=\emptyset$.

Proof. Take an embedding of $G$ in a disc such that $a, b, c, d$ occur in clockwise order on the boundary of the disc. Let $x, y \in N_{G}(b)-\{a, c\}$ such that $x, y$ is $\left(T_{a}, T_{c}\right)$-ordered (see Figure 8). Hence, $T_{a}[a, x] \cap T_{c}[c, y]=\emptyset$. Since $T_{a}$ and $T_{d}$ are independent, $P:=T_{a}[a, x] \cup T_{d}[d, x]$ is an $a-d$ path in $G-\{a, c\}$, and $P$ divides the disc into two closed regions $B$ and $C$, with $b$ in $B$. By planarity and since $T_{a}[a, x] \cap T_{c}[c, y]=\emptyset, T_{d}[d, y]$ lies in $B$. Then by planarity $T_{a}[a, x] \cap T_{d}[d, y]=\emptyset$.

(3.7) Lemma. Let $(G, a, b, c, d)$ be a planar graph, and let $\left\{T_{a}, T_{b}, T_{c}, T_{d}\right\}$ be an independent spanning $\{a, b, c, d\}$-system of $G$, where $T_{v}$ is rooted at $v$ for each $v \in\{a, b, c, d\}$. Assume that $b$ has at least three neighbors in $V(G)-\{a, c\}$. Then for any $\left(T_{a}, T_{c}\right)$-ordered triple $x, y, z \in$ $N_{G}(b)-\{a, c\}, T_{a}[a, x], T_{d}[d, y]$ and $T_{c}[c, z]$ are pairwise disjoint. 


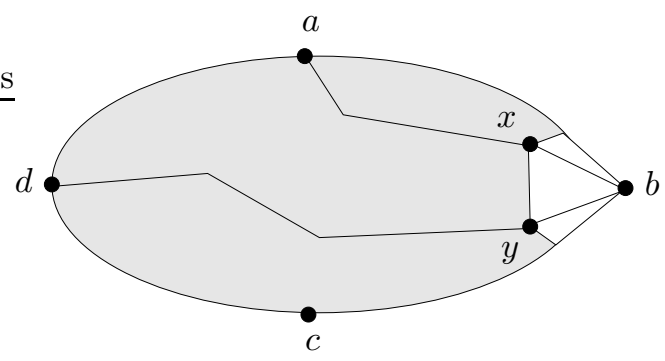

Figure 8: Two disjoint paths, one in $T_{a}$ and the other in $T_{d}$.

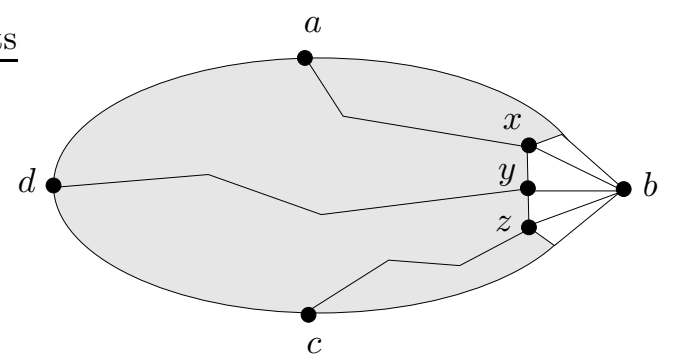

Figure 9: Three disjoint paths contained in $T_{a}, T_{c}$ and $T_{d}$, respectively.

Proof. Take an embedding of $G$ in a disc such that $a, b, c, d$ occur in clockwise order on the boundary of the disc. Let $x, y, z \in N_{G}(b)$ such that $x, y, z$ is $\left(T_{a}, T_{c}\right)$-ordered (see Figure 9). Hence, $T_{a}[a, x] \cap T_{c}[c, y]=\emptyset$ and $T_{a}[a, y] \cap T_{c}[c, z]=\emptyset$.

By Lemma (3.4), $T_{a}[a, x] \cap T_{c}[c, z]=\emptyset$. Hence, the path $P:=T_{d}[d, y]+\{b, y b\}$ divides the disc into closed regions $A$ and $C$, with $T_{a}[a, x]$ in $A$ and $T_{c}[c, z]$ in $C$. By (3.6), $T_{a}[a, x] \cap T_{d}[d, y]=\emptyset$. By applying a mirror image version of (3.6) we can show that $T_{d}[d, y] \cap T_{c}[c, z]=\emptyset$.

\section{Numberings}

By Theorem (2.8) $G$ has a non-separating chain decomposition rooted at $r$. In this section we will combine this decomposition with Theorem (3.2) to produce a numbering of a subset of $V(G)$. This numbering will be used in the next section to construct four independent spanning trees rooted at $r$.

In the rest of this section we fix the following notation.

(4.1) Notation. Let $G$ be a 4-connected graph and let $r \in V(G)$. Fix a non-separating chain decomposition of $G$ rooted at $r$, say $\mathcal{C}:=\left(H_{1}, \ldots, H_{t}\right), t \geq 2$. Define the sequences $G_{0}, G_{1}, \ldots, G_{t-1}$ and $\bar{G}_{1}, \ldots, \bar{G}_{t}$ as follows.

(i) $G_{0}:=\bar{G}_{t}:=(\{r\}, \emptyset)$, and

(ii) for $i=1, \ldots, t-1, G_{i}:=G\left[\bigcup_{j=1}^{i} I\left(H_{j}\right)\right]$ and $\bar{G}_{i}:=G-\left(V\left(G_{i}\right)-\{r\}\right)$.

(4.2) Notation. Suppose that $H_{i}(1 \leq i \leq t)$ is an up $G_{i-1}$-chain in $G$ or a down $G_{i-1}$-chain in $G$ in $G$. Let $H_{i}:=v_{0} B_{1} v_{1} B_{2} v_{2} \ldots v_{k-1} B_{k} v_{k}$. For each 2-connected $B_{j}$ there exist $u_{j}, w_{j}$ (both on $V\left(G_{i-1}\right)$ or both on $\left.V\left(\bar{G}_{i}\right)\right)$ such that $B_{j}-\left\{v_{j-1}, v_{j}\right\}$ is a component of $G-\left\{v_{j-1}, v_{j}, u_{j}, w_{j}\right\}$, and $\left.\left(B_{j}^{+}, v_{j-1}, u_{j}, v_{j}, w_{j}\right\}\right)$ is planar, where $B_{j}^{+}:=G\left[V\left(B_{j}\right) \cup\left\{u_{j}, w_{j}\right\}\right]-u_{j} w_{j}$. We refer to each such $B_{j}^{+}$as a planar section in $\mathcal{C}$. The vertices $v_{j-1}, v_{j}, u_{j}, w_{j}$ are the terminals of $B_{j}^{+}$. See Figure 10 for an illustration. Note that the notation above depends on $i$. For the sake of clarity 
we will not make it explicit in the notation, but whenever we use this we will make clear which $i$ we refer to. Furthermore, the algorithms we will describe deal with each $H_{i}$ separately, and thus, no confusion should arise.
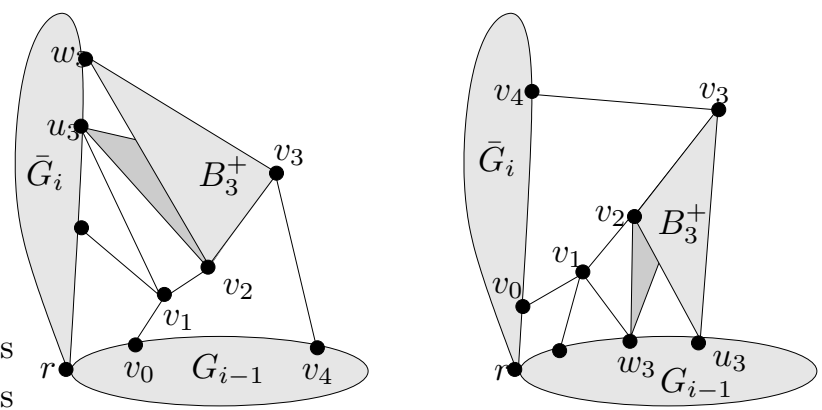

Figure 10: $v_{2}, v_{3}, u_{3}, w_{3}$ are the terminals of $B_{3}^{+}$.

(4.3) Definition. Suppose that $H_{i}(1 \leq i \leq t)$ is a triangle $G_{i-1}$-chain in $G$. See Figure 4 . Let $I\left(H_{i}\right):=\left\{v_{1}, v_{2}, v_{3}\right\}$, let $V\left(H_{i}\right)-I\left(H_{i}\right):=\left\{x_{1}, x_{2}, x_{3}\right\}$, and suppose that $x_{j} v_{j} \in E(G)$ for $j=1,2,3$. We say that $v_{j} x_{j}(j=1,2,3)$ are the legs of $H_{i}$.

(4.4) Definition. Let $D \subseteq V(G)$. A numbering of $D$ is a function from $D$ to $\{1, \ldots,|D|\}$. Let $g$ be a numbering of $D$, let $v_{1}, \ldots, v_{k}$ be a sequence of distinct vertices in $V(G)-D$, and let $v_{0} \in D$. The extension $g^{\prime}$ of $g$ to $v_{1}, \ldots, v_{k}$ from $v_{0}$ is defined as follows:

(a) for $1 \leq i \leq k$, let $g^{\prime}\left(v_{i}\right):=g\left(v_{0}\right)+i$,

(b) for each $v \in D$ with $g(v) \leq g\left(v_{0}\right)$ let $g^{\prime}(v)=g(v)$, and

(c) for each $v \in D$ with $g(v)>g\left(v_{0}\right)$ let $g^{\prime}(v):=g(v)+k$.

Note that $g^{\prime}$ is a numbering of $D \cup\left\{v_{1}, \ldots, v_{k}\right\}$. For convenience, if $D \subseteq V(G)$ and $\sigma$ denotes a sequence $v_{1}, \ldots, v_{k}$ of vertices in $V(G)-D$, we let $D \cup\{\sigma\}:=D \cup\left\{v_{1}, \ldots, v_{k}\right\}$.

In order to compute the desired numberings $g$ and $f$ from a non-separating chain decomposition, we need to find independent spanning systems in the planar sections in $\mathcal{C}$.

(4.5) Assumption. For each planar section $B_{j}^{+}$in $\mathcal{C}$, with terminals $v_{j-1}, v_{j}, u_{j}, w_{j}$, we compute an independent spanning $\left\{v_{j-1}, v_{j}, u_{j}, w_{j}\right\}$-system of $B_{j}^{+}$. By Theorem (3.2), such a system can be computed in $O\left(\left|V\left(B_{j}^{+}\right)\right|+\left|E\left(B_{j}^{+}\right)\right|\right)$time. Since two distinct planar sections are edge-disjoint, the overall time consumed in this phase (for all planar sections in $\mathcal{C}$ ) is $O(|V(G)|+|E(G)|$ ).

Next we describe the algorithm for computing a numbering $g$ of a subset of $V(G)$. It also computes a sequence $\{r\}=D_{0} \subset D_{1} \subset \ldots \subset D_{t-1}$ of subsets of $V(G)$ such that for $i=1, \ldots, t$, $N_{G}\left(H_{i}\right) \cap V\left(G_{i-1}\right) \subseteq D_{i-1}$. When the algorithm stops, $g$ is a numbering of $D_{t-1}$. We note that keeping track of this sequence is not necessary for computing $g$, but its inclusion will make proofs in Section 6.

\section{Algorithm Numbering $g$.}

Description: The algorithm executes $t-1$ iterations, where $t$ is the number of chains in $\mathcal{C}$. At the beginning of the first iteration we have $i=1, D_{0}:=\{r\}$, and $g(r):=1$. At the beginning of each iteration, we have an integer $i$ with $1 \leq i \leq t-1$, a subset $D_{i-1} \subseteq V\left(G_{i-1}\right)$ such that $N_{G}\left(H_{i}\right) \cap V\left(G_{i-1}\right) \subseteq D_{i-1}$, and a numbering $g$ of $D_{i-1}$. 
Each iteration consists of the following: update $g$ and define $D_{i}$ according to the following cases (depending on the type of $H_{i}$ ), and if $i<t-1$, then set $i \leftarrow i+1$ and start a new iteration.

Case 1: $H_{i}$ is an elementary $G_{i-1}$-chain in $G$.

Let $H_{i}:=v_{0} B_{1} v_{1} B_{2} v_{2}$ and assume that $v_{0}, v_{2}$ are labeled so that $g\left(v_{0}\right)<g\left(v_{2}\right)$.

Extend $g$ to $v_{1}$ from $v_{0}$, and let $D_{i}:=D_{i-1} \cup\left\{v_{1}\right\}$.

Case 2: $i=1$ or $H_{i}$ is an up $G_{i-1}$-chain in $G$, but not an elementary $G_{i-1}$-chain.

Let $H_{i}:=v_{0} B_{1} v_{1} \ldots v_{k-1} B_{k} v_{k}$ and suppose that $v_{0}, \ldots, v_{k}$ and $B_{1}, \ldots, B_{k}$ are labelled so that $v_{0}=v_{k}=r$ when $i=1$, and $g\left(v_{0}\right)<g\left(v_{k}\right)$ when $i \neq 1$. For each 2-connected $B_{j}$, let $u_{j}, w_{j}$ denote the terminals of $B_{j}^{+}$other than $v_{j-1}, v_{j}$. Let $T_{v_{j-1}}^{j}, T_{v_{j}}^{j}$ denote the trees rooted respectively at $v_{j-1}, v_{j}$ in the independent spanning $\left\{v_{j-1}, v_{j}, u_{j}, w_{j}\right\}$-system of $B_{j}^{+}$computed in (4.5).

For each $j=1, \ldots, k$, compute a sequence $\sigma_{j}$ as follows. If $B_{j}$ is 2-connected, then let $\sigma_{j}$ be a $\left(T_{v_{j-1}}^{j}, T_{v_{j}}^{j}\right)$-ordering of $N_{B_{j}^{+}}\left(\left\{u_{j}, w_{j}\right\}\right)-\left\{v_{j-1}, v_{j}\right\}$ (the existence of this ordering is guaranteed by (3.5)). If $B_{j}$ is trivial, then let $\sigma_{j}$ denote the empty sequence.

Extend $g$ to $\sigma:=\sigma_{1}, v_{1}, \sigma_{2}, v_{2}, \ldots, v_{k-1}, \sigma_{k}$ from $v_{0}$, and let $D_{i}:=D_{i-1} \cup\{\sigma\}$. See Figure 11 for an illustration.

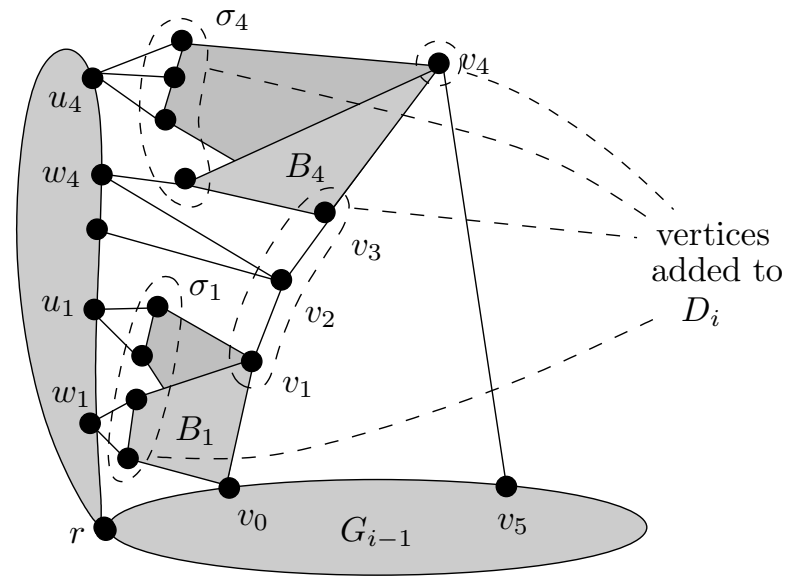

Figure 11: Extending the numbering $g$ to an up $G_{i-1}$-chain.

Case 3: $H_{i}$ is a down $G_{i-1}$-chain in $G$, but not an elementary $G_{i-1}$-chain.

Let $H_{i}:=v_{0} B_{1} v_{1} \ldots v_{k-1} B_{k} v_{k}$. For each 2-connected block $B_{j}$ let $u_{j}, w_{j}$ denote the terminals of $B_{j}^{+}$other than $v_{j-1}, v_{j}$ with $g\left(u_{j}\right)<g\left(w_{j}\right)$. Let $T_{u_{j}}^{j}, T_{w_{j}}^{j}$ denote trees rooted respectively at $u_{j}, w_{j}$ in the independent spanning $\left\{v_{j-1}, v_{j}, u_{j}, w_{j}\right\}$-system of $B_{j}^{+}$computed in (4.5).

Let $D_{i}:=D_{i-1} \cup N_{B_{1}}\left(v_{0}\right) \cup N_{B_{k}}\left(v_{k}\right)$. See Figure 12 for an illustration. Extend $g$ according to the following three subcases.

Subcase 3.1: $k=1$ (thus, $B_{1}$ is 2-connected).

Let $\sigma$ denote a $\left(T_{u_{1}}^{1}, T_{w_{1}}^{1}\right)$-ordering of $N_{B_{1}^{+}}\left(\left\{v_{0}, v_{1}\right\}\right)-\left\{u_{1}, w_{1}\right\}=N_{B_{1}}\left(v_{0}\right) \cup N_{B_{k}}\left(v_{k}\right)$ (the existence of this ordering is guaranteed by (3.5)). Extend $g$ to $\sigma$ from $u_{1}$.

Subcase 3.2: $k=2$, and $B_{1}$ or $B_{2}$ is trivial.

Note that since $H_{i}$ is not an elementary $G_{i-1}$-chain, $B_{1}$ or $B_{2}$ is nontrivial.

Assume then (renaming $B_{1}$ and $B_{2}$ if necessary) that $B_{1}$ is 2 -connected and $B_{2}$ is trivial. Extend $g$ according to the following cases. 


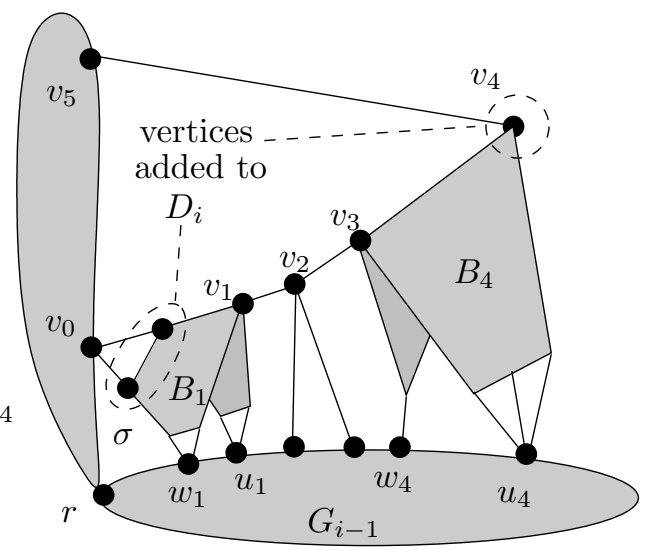

Figure 12: Extending the numbering $g$ to a down $G_{i-1}$-chain.

- $v_{1}$ has no neighbor in $V\left(G_{i-1}\right)$. Let $q_{1}, q_{2}, q_{3}$ be neighbors of $v_{1}$ in $B_{1}$ (they exist since $G$ is 4-connected), and assume that $q_{1}, q_{2}, q_{3}$ is $\left(T_{u_{1}}^{1}, T_{w_{1}}^{1}\right)$-ordered (this is possible by (3.4)). By Lemma (3.7), $T_{u_{1}}^{1}\left[u_{1}, q_{1}\right], T_{v_{0}}^{1}\left[v_{0}, q_{2}\right]$ and $T_{w_{1}}^{1}\left[w_{1}, q_{3}\right]$ are disjoint. Let $H:=B_{1}^{+} \cup B_{2}$ (note that $H-\left\{v_{0}, v_{2}, u_{1}, w_{1}\right\}$ is a component of $\left.G-\left\{v_{0}, v_{2}, u_{1}, w_{1}\right\}\right)$. Then $\left(H, v_{0}, u_{1}, v_{2}, w_{1}\right)$ is planar, and $\left\{T_{v_{0}}^{1}+\left\{v_{1}, v_{1} q_{2}\right\}, T_{v_{1}}^{1}+\left\{v_{2}, v_{1} v_{2}\right\}, T_{u_{1}}^{1}+\left\{v_{1}, v_{1} q_{1}\right\}, T_{w_{1}}^{1}+\left\{v_{1}, v_{1} q_{3}\right\}\right\}$ forms an independent spanning $\left\{v_{0}, u_{1}, v_{2}, w_{1}\right\}$-system of $H$.

Let $\sigma$ denote a $\left(T_{u_{1}}^{1}+\left\{v_{1}, v_{1} q_{1}\right\}, T_{w_{1}}^{1}+\left\{v_{1}, v_{1} q_{3}\right\}\right)$-ordering of $N_{H}\left(\left\{v_{0}, v_{2}\right\}\right)-\left\{u_{1}, w_{1}\right\}$ (the existence of this ordering is guaranteed by (3.5)). Extend $g$ to $\sigma$ from $u_{1}$.

Comment: we also keep track of $q_{1}, q_{2}, q_{3}$ for the construction of the independent spanning trees.

- $v_{1}$ has a neighbor in $V\left(G_{i-1}\right)$. Let $x \in N_{G}\left(v_{1}\right) \cap V\left(G_{i-1}\right)$ with $g(x)$ minimum, and let $\sigma$ denote a $\left(T_{u_{1}}^{1}, T_{w_{1}}^{1}\right)$-ordering of $N_{B_{1}^{+}}\left(v_{0}\right)-\left\{u_{1}, w_{1}\right\}$ (the existence of this ordering is guaranteed by (3.4)). If $g(x)>g\left(u_{1}\right)$, then extend $g$ to $\sigma, v_{1}$ from $u_{1}$, where $\sigma, v_{1}$ is the sequence obtained from $\sigma$ by adding $v_{1}$ at the end. If $g(x) \leq g\left(u_{1}\right)$, then extend $g$ to $v_{1}, \sigma$ from $x$, where $v_{1}, \sigma$ is the sequence obtained from $\sigma$ by adding $v_{1}$ in the front.

Subcase 3.3: $k \geq 3$, or $k=2$ and both $B_{1}, B_{2}$ are 2-connected.

Extend $g$ to $N_{B_{1}}\left(v_{0}\right)$ according to the following cases.

- $B_{1}$ is 2-connected. Let $\sigma$ denote a $\left(T_{u_{1}}^{1}, T_{w_{1}}^{1}\right)$-ordering of $N_{B_{1}^{+}}\left(v_{0}\right)-\left\{u_{1}, w_{1}\right\}=N_{B_{1}}\left(v_{0}\right)$ (the existence of this ordering is guaranteed by (3.4)). Extend $g$ to $\sigma$ from $u_{1}$.

- Both $B_{1}$ and $B_{2}$ are trivial. Let $x \in N_{G}\left(v_{1}\right) \cap V\left(G_{i-1}\right)$ with $g(x)$ minimum. Extend $g$ to $v_{1}$ from $x$.

- $B_{1}$ is trivial and $B_{2}$ is 2-connected.

- If $v_{1}$ has no neighbor in $V\left(G_{i-1}\right)$, extend $g$ to $v_{1}$ from $u_{2}$.

- If $v_{1}$ has a neighbor in $V\left(G_{i-1}\right)$, let $x \in N_{G}\left(v_{1}\right) \cap V\left(G_{i-1}\right)$ with $g(x)$ minimum. If $g(x)>g\left(u_{2}\right)$ then extend $g$ to $v_{1}$ from $u_{2}$. If $g(x) \leq g\left(u_{2}\right)$, then extend $g$ to $v_{1}$ from $x$.

Extend (the resulting) $g$ to $N_{B_{k}}\left(v_{k}\right)$ according to the following cases.

- $B_{k}$ is 2 -connected. Let $\sigma$ be a $\left(T_{u_{k}}^{k}, T_{w_{k}}^{k}\right)$-ordering of $N_{B_{k}^{+}}\left(v_{k}\right)-\left\{u_{k}, w_{k}\right\}=N_{B_{k}}\left(v_{k}\right)$ (the existence of this ordering is guaranteed by (3.4)). Extend $g$ to $\sigma$ from $u_{k}$. 
- Both $B_{k}$ and $B_{k-1}$ are trivial. Let $x \in N_{G}\left(v_{k-1}\right) \cap V\left(G_{i-1}\right)$ with $g(x)$ minimum. Extend $g$ to $v_{k-1}$ from $x$.

- $B_{k}$ is trivial and $B_{k-1}$ is 2-connected.

- If $v_{k-1}$ has no neighbor in $V\left(G_{i-1}\right)$, extend $g$ to $v_{k-1}$ from $u_{k-1}$.

- If $v_{k-1}$ has a neighbor in $V\left(G_{i-1}\right)$, let $x \in N_{G}\left(v_{k-1}\right) \cap V\left(G_{i-1}\right)$ with $g(x)$ minimum. If $g(x)>g\left(u_{k-1}\right)$ then extend $g$ to $v_{k-1}$ from $u_{k-1}$. If $g(x) \leq g\left(u_{k-1}\right)$, then extend $g$ to $v_{k-1}$ from $x$.

Case 4: $H_{i}$ is a triangle $G_{i-1}$-chain in $G$.

Let $I\left(H_{i}\right):=\left\{v_{1}, v_{2}, v_{3}\right\}$, and let $v_{j} x_{j}(j=1,2,3)$ be the legs of $H_{i}$. Suppose that $v_{1}, v_{2}, v_{3}$ are labelled so that $g\left(x_{1}\right)<g\left(x_{2}\right)<g\left(x_{3}\right)$. Let $D_{i}:=D_{i-1} \cup\left\{v_{1}, v_{2}, v_{3}\right\}$. Extend $g$ to $v_{1}, v_{2}, v_{3}$ from $x_{2}$.

This concludes the description of the algorithm for computing $g$.

(4.6) Lemma. Algorithm Numbering g runs in $O\left(|V(G)|^{3}\right)$ time.

Proof. Observe that at the $i^{\text {th }}$ iteration, Algorithm Numbering $g$ extends the current numbering $g^{i}$ to a sequence $\sigma$ from a previously numbered vertex $v \in D_{i-1}$. Clearly, given $g^{i}, \sigma$, and $v$, this extension can be computed in $O(|V(G)|)$ time. We now analyze the time spent at each iteration of Algorithm Numbering $g$ according to Cases 1-4. We use the same notation as in the algorithm.

If Case 1 occurs $\left(H_{i}\right.$ is an elementary $G_{i-1}$-chain in $\left.G\right)$, then Algorithm Numbering $g$ extends $g$ to $v_{1}$, This can be done in $O(|V(G)|)$ time.

If Case 2 occurs ( $H_{i}$ is an up $G_{i-1}$-chain), then Algorithm Numbering $g$ computes sequences $\sigma_{1}, \ldots, \sigma_{k}$, where $\sigma_{j}$ denotes the empty sequence when $B_{j}$ is trivial, and $\sigma_{j}$ is a $\left(T_{v_{j-1}}^{j}, T_{v_{j}}^{j}\right)$ ordering of $N_{B_{j}^{+}}\left(\left\{u_{j}, w_{j}\right\}\right)-\left\{v_{j-1}, v_{j}\right\}$ when $B_{j}$ is 2-connected. In the latter case, by Lemma (3.5) the sequence $\sigma_{j}$ can be computed in $O\left(\left|V\left(B_{j}^{+}\right)\right|^{2}\right)$ time. Thus, the algorithm spends $O\left(|V(G)|^{2}\right)$ time to compute $\sigma_{1}, \ldots, \sigma_{k}$. After that, the algorithm extends $g$ to $v_{0}, \sigma_{1}, v_{1}, \ldots, v_{k-1}, \sigma_{k}, v_{k}$, which can be done in $O(|V(G)|)$ time. Therefore, the algorithm spends $O\left(|V(G)|^{2}\right)$ time if Case 2 occurs.

If Case 3 occurs ( $H_{i}$ is a down $G_{i-1}$-chain, but not an elementary $G_{i-1}$-chain), then Algorithm Numbering $g$ considers three cases.

- If Subcase 3.1 occurs $(k=1)$, then the algorithm computes a $\left(T_{u_{1}}^{1}, T_{w_{1}}^{1}\right)$-ordering $\sigma$ of $N_{B_{1}^{+}}\left(\left\{v_{0}, v_{1}\right\}\right)-\left\{u_{1}, w_{1}\right\}$, and extends $g$ to $\sigma$ from $u_{1}$. The sequence $\sigma$ can be computed in $O\left(|V(G)|^{2}\right)$ time by Lemma (3.5), and the extension of $g$ can be computed in $O(|V(G)|)$ time, resulting in $O\left(|V(G)|^{2}\right)$ time for this iteration.

- If Subcase 3.2 occurs $\left(k=2\right.$, and $B_{1}$ or $B_{2}$ is trivial), then the algorithm considers two subcases, according to whether or not $v_{1}$ has a neighbor in $V\left(G_{i-1}\right)$.

- If $v_{1}$ has no neighbor in $V\left(G_{i-1}\right)$, the algorithm chooses neighbors $q_{1}, q_{2}, q_{3}$ of $v_{1}$ in $B_{1}$ and computes a $\left(T_{u_{1}}^{1}+\left\{v_{1}, v_{1} q_{1}\right\}, T_{w_{1}}^{1}+\left\{v_{1}, v_{1} q_{3}\right\}\right)$-ordering $\sigma$ of $N_{B_{1}}\left(v_{0}\right) \cup N_{B_{k}}\left(v_{k}\right)=$ $N_{H}\left(\left\{v_{0}, v_{2}\right\}\right)-\left\{u_{1}, w_{1}\right\}$ as in Subcase 3.1, and extends $g$ to $\sigma$ from $u_{1}$. Thus, the algorithm spends $O\left(|V(G)|^{2}\right)$ time in this case.

- If $v_{1}$ has a neighbor in $V\left(G_{i-1}\right)$, then the algorithm computes a $\left(T_{u_{1}}^{1}, T_{w_{1}}^{1}\right)$-ordering $\sigma$ of $N_{B_{1}^{+}}\left(v_{0}\right)-\left\{u_{1}, w_{1}\right\}$, and it performs an extension on $g$. The sequence $\sigma$ can be computed in $O(|V(G)|)$ time by Lemma (3.4), and the extensions can be computed in $O(|V(G)|)$ time. Thus, the algorithm spends $O(|V(G)|)$ time in this case. 
- If Subcase 3.3 occurs ( $k \geq 3$, or, $k=2$ and both $B_{1}, B_{2}$ are 2 -connected), then the algorithm extends $g$ to $N_{B_{1}}\left(v_{0}\right)$ and extends $g$ to $N_{B_{k}}\left(v_{k}\right)$. The algorithm may need to compute a $\left(T_{u_{1}}^{1}, T_{w_{1}}^{1}\right)$-ordering of $N_{B_{1}^{+}}\left(v_{0}\right)-\left\{u_{1}, w_{1}\right\}$ and a $\left(T_{u_{k}}^{k}, T_{w_{k}}^{k}\right)$-ordering of $N_{B_{k}^{+}}\left(v_{k}\right)-\left\{u_{k}, w_{k}\right\}$ but both can be done in $O(|V(G)|)$ time by Lemma (3.4). It is not hard to check that the algorithm spends $O(|V(G)|)$ time in this case.

If Case 4 occurs $\left(H_{i}\right.$ is a triangle chain), then Algorithm Numbering $g$ extends $g$ to $v_{1}, v_{2}, v_{3}$. This can be done in $O(|V(G)|)$ time.

From the analysis above, it follows that Algorithm Numbering $g$ spends $O\left(|V(G)|^{2}\right)$ time in each iteration. Since the number of iterations is $t<n$, the numbering $g$ can be computed in $O\left(|V(G)|^{3}\right)$ time.

Note that the extension operation does not affect the order of the vertices previously numbered, although their actual $g$ values may have changed. Thus, at each iteration the algorithm orders the vertices in $D_{i}-D_{i-1}$ without affecting the order of the vertices in $D_{i-1}$. In fact, it does not affect the order of the vertices in $D_{j}$ for every $1 \leq j \leq i-1$.

The numbering $g$ will be used to construct two independent spanning trees rooted at $r$ from $\mathcal{C}=\left(H_{1}, \ldots, H_{t}\right)$, in order from $H_{1}$ to $H_{t}$. For constructing the other two spanning trees we compute a numbering $f$ by examining the chains of $\mathcal{C}$ in reverse order.

Let $\mathcal{C}^{\prime}:=\left(H_{t}, H_{t-1}, \ldots, H_{1}\right)$. Note that if there exists no elementary chain or triangle chain in $\mathcal{C}$, then $\mathcal{C}^{\prime}$ would be a non-separating chain decomposition of $G$, but with the roles of up chain and down chain switched. More precisely, an up $G_{i-1}$-chain in the decomposition $\mathcal{C}$ is a down $\bar{G}_{i}$-chain in the decomposition $\mathcal{C}^{\prime}$, and a down $G_{i-1}$-chain in the decomposition $\mathcal{C}$ is an up $\bar{G}_{i}$-chain in the decomposition $\mathcal{C}^{\prime}$.

The algorithm for computing $f$ is analogous to Algorithm Numbering $g$ when it deals with an up $G_{i-1}$-chain or a down $G_{i-1}$-chain. The differences appear when it deals with an elementary $G_{i-1}$-chain or a triangle $G_{i-1}$-chain. The algorithm also computes a sequence $\{r\}=D_{t+1}^{\prime} \subset$ $D_{t}^{\prime} \subset \ldots \subset D_{2}^{\prime}$ of subsets of $V(G)$ such that for $t \geq i \geq 1, N_{G}\left(H_{i}\right) \cap V\left(\bar{G}_{i}\right) \subseteq D_{i+1}^{\prime}$.

\section{Algorithm Numbering $f$.}

Description: The algorithm executes $t-1$ iterations, where $t$ is the number of chains in $\mathcal{C}^{\prime}:=$ $\left(H_{t}, \ldots, H_{1}\right)$. At the beginning of the first iteration we have $i=t, D_{t+1}^{\prime}:=\{r\}$, and $f(r):=1$. At the beginning of each iteration, we have an integer $i$ with $t \geq i \geq 2$, a subset $D_{i+1}^{\prime} \subseteq V\left(\bar{G}_{i}\right)$ such that $N_{G}\left(H_{i}\right) \cap V\left(\bar{G}_{i}\right) \subseteq D_{i+1}^{\prime}$, and a numbering $f$ of $D_{i}^{\prime}$.

Each iteration consists of the following: update $f$ and define $D_{i}^{\prime}$ according to the following cases (depending on the chain type of $H_{i}$ ), and, if $i>2$, then set $i \leftarrow i-1$ and start a new iteration.

Case 1: $H_{i}$ is an elementary $G_{i-1}$-chain in $G$.

Let $H_{i}:=v_{0} B_{1} v_{1} B_{2} v_{2}$ and let $v_{0}^{\prime}, v_{2}^{\prime}$ be neighbors of $v_{1}$ in $V\left(\bar{G}_{i}\right)$ with $f\left(v_{0}^{\prime}\right)<f\left(v_{2}^{\prime}\right)$. Extend $f$ to $v_{1}$ from $v_{0}^{\prime}$, and let $D_{i}^{\prime}:=D_{i+1}^{\prime} \cup\left\{v_{1}\right\}$.

Case 2: $i=t$ or $H_{i}$ is a down $G_{i-1}$-chain in $G$, but not an elementary $G_{i-1}$-chain.

Let $H_{i}:=v_{0} B_{1} v_{1} \ldots v_{k-1} B_{k} v_{k}$ and suppose that $v_{0}, \ldots, v_{k}$ and $B_{1}, \ldots, B_{k}$ are labelled so that $v_{0}=v_{k}=r$ when $i=t$, and $f\left(v_{0}\right)<f\left(v_{k}\right)$ when $i \neq t$. For each 2-connected $B_{j}$, let $u_{j}, w_{j}$ be the terminals of $B_{j}^{+}$other than $v_{j-1}, v_{j}$. Let $T_{v_{j-1}}^{j}, T_{v_{j}}^{j}$ denote the trees rooted respectively at $v_{j-1}, v_{j}$ in the independent spanning $\left\{v_{j-1}, v_{j}, u_{j}, w_{j}\right\}$-system of $B_{j}^{+}$computed in (4.5).

For each $j=1, \ldots, k$ compute a sequence $\sigma_{j}$ as follows. If $B_{j}$ is 2-connected, then let $\sigma_{j}$ be a $\left(T_{v_{j-1}}^{j}, T_{v_{j}}^{j}\right)$-ordering of $N_{B_{j}^{+}}\left(\left\{u_{j}, w_{j}\right\}\right)-\left\{v_{j-1}, v_{j}\right\}$ (the existence of this ordering is guaranteed by (3.5)). If $B_{j}$ is trivial, then let $\sigma_{j}$ denote the empty sequence.

Extend $f$ to $\sigma:=\sigma_{1}, v_{1}, \sigma_{2}, v_{2}, \ldots, v_{k-1}, \sigma_{k}$ from $v_{0}$, and let $D_{i}^{\prime}:=D_{i+1}^{\prime} \cup\{\sigma\}$. See Figure 13 for an illustration. 


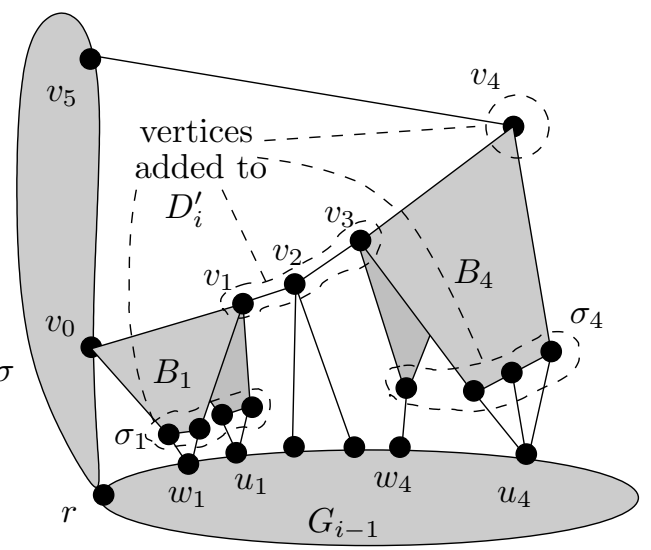

Figure 13: Extending the numbering $f$ to a down $G_{i-1}$-chain.

Case 3: $H_{i}$ is an up $G_{i-1}$-chain in $G$, but not an elementary $G_{i-1}$-chain in $G$.

Let $H_{i}:=v_{0} B_{1} v_{1} \ldots v_{k-1} B_{k} v_{k}$. For each 2-connected $B_{j}$, let $u_{j}, w_{j}$ denote the terminals of $B_{j}^{+}$other than $v_{j-1}, v_{j}$, with $f\left(u_{j}\right)<f\left(w_{j}\right)$. Let $T_{u_{j}}^{j}, T_{w_{j}}^{j}$ denote the trees rooted respectively at $u_{j}, w_{j}$ in the independent spanning $\left\{v_{j-1}, v_{j}, u_{j}, w_{j}\right\}$-system of $B_{j}^{+}$computed in (4.5).

Let $D_{i}^{\prime}:=D_{i+1}^{\prime} \cup N_{B_{1}}\left(v_{0}\right) \cup N_{B_{k}}\left(v_{k}\right)$. See Figure 14 for an illustration. Extend $f$ according to the following three subcases.

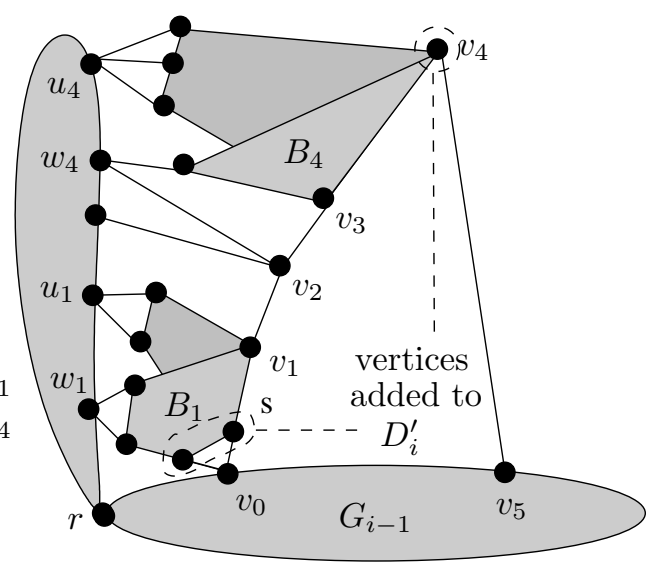

Figure 14: Extending the numbering $f$ to an up $G_{i-1}$-chain.

Subcase 3.1: $k=1$ (thus, $B_{1}$ is 2 -connected).

Let $\sigma$ denote a $\left(T_{u_{1}}^{1}, T_{w_{1}}^{1}\right)$-ordering of $N_{B_{1}^{+}}\left(\left\{v_{0}, v_{1}\right\}\right)-\left\{u_{1}, w_{1}\right\}=N_{B_{1}}\left(v_{0}\right) \cup N_{B_{k}}\left(v_{k}\right)$ (the existence of this ordering is guaranteed by (3.5)). Extend $f$ to $\sigma$ from $u_{1}$.

Subcase 3.2: $k=2$, and, $B_{1}$ or $B_{2}$ is trivial.

Note that since $H_{i}$ is not an elementary chain, $B_{1}$ or $B_{2}$ is nontrivial.

Assume then (renaming $B_{1}$ and $B_{2}$ if necessary) that $B_{1}$ is 2 -connected and $B_{2}$ is trivial. Extend $f$ according to the following cases.

- $v_{1}$ has no neighbor in $V\left(\bar{G}_{i}\right)$. Let $q_{1}, q_{2}, q_{3}$ be distinct neighbors of $v_{1}$ in $B_{1}$ (they exist since $G$ is 4-connected), and assume that $q_{1}, q_{2}, q_{3}$ is $\left(T_{u_{1}}^{1}, T_{w_{1}}^{1}\right.$ )-ordered (this is possible by (3.4)). By Lemma (3.7), $T_{u_{1}}^{1}\left[u_{1}, q_{1}\right], T_{v_{0}}^{1}\left[v_{0}, q_{2}\right]$ and $T_{w_{1}}^{1}\left[w_{1}, q_{3}\right]$ are disjoint. Let $H:=B_{1}^{+} \cup B_{2}$. 
Note that $H-\left\{v_{0}, v_{2}, u_{1}, w_{1}\right\}$ is a component of $G-\left\{v_{0}, v_{2}, u_{1}, w_{1}\right\},\left(H, v_{0}, u_{1}, v_{2}, w_{1}\right)$ is planar, and $\left\{T_{v_{0}}^{1}+\left\{v_{1}, v_{1} q_{2}\right\}, T_{v_{1}}^{1}+\left\{v_{2}, v_{1} v_{2}\right\}, T_{u_{1}}^{1}+\left\{v_{1}, v_{1} q_{1}\right\}, T_{w_{1}}^{1}+\left\{v_{1}, v_{1} q_{3}\right\}\right\}$ is an independent spanning $\left\{v_{0}, v_{2}, u_{1}, w_{1}\right\}$-system of $H$.

Let $\sigma$ denote a $\left(T_{u_{1}}^{1}+\left\{v_{1}, v_{1} q_{1}\right\}, T_{w_{1}}^{1}+\left\{v_{1}, v_{1} q_{3}\right\}\right)$-ordering of $N_{H}\left(\left\{v_{0}, v_{2}\right\}\right)-\left\{u_{1}, w_{1}\right\}=$ $N_{B_{1}}\left(v_{0}\right) \cup N_{B_{k}}\left(v_{k}\right)$ (the existence of this ordering is guaranteed by (3.5)). Extend $f$ to $\sigma$ from $u_{1}$.

Comment: we also keep track of $q_{1}, q_{2}, q_{3}$ for the construction of the independent spanning trees.

- $v_{1}$ has a neighbor in $V\left(\bar{G}_{i}\right)$. Let $x \in N_{G}\left(v_{1}\right) \cap V\left(\bar{G}_{i}\right)$ with $f(x)$ minimum, and let $\sigma$ denote a $\left(T_{u_{1}}^{1}, T_{w_{1}}^{1}\right.$ )-ordering of $N_{B_{1}}\left(v_{0}\right)=N_{B_{1}^{+}}\left(v_{0}\right)-\left\{u_{1}, w_{1}\right\}$ (the existence of this ordering is guaranteed by (3.4)). If $f(x)>f\left(u_{1}\right)$, then extend $f$ to $\sigma, v_{1}$ from $u_{1}$. If $f(x) \leq f\left(u_{1}\right)$, then extend $f$ to $v_{1}, \sigma$ from $x$.

Subcase 3.3: $k \geq 3$, or, $k=2$ and both $B_{1}, B_{2}$ are 2-connected.

Extend $f$ to $N_{B_{1}}\left(v_{0}\right)$ according to the following cases.

- $B_{1}$ is 2-connected. Let $\sigma$ denote a $\left(T_{u_{1}}^{1}, T_{w_{1}}^{1}\right)$-ordering of $N_{B_{1}}\left(v_{0}\right)=N_{B_{1}^{+}}\left(v_{0}\right)-\left\{u_{1}, w_{1}\right\}$ (the existence of this ordering is guaranteed by (3.4)). Extend $f$ to $\sigma$ from $u_{1}$.

- Both $B_{1}$ and $B_{2}$ are trivial. Let $x \in N_{G}\left(v_{1}\right) \cap V\left(\bar{G}_{i}\right)$ with $f(x)$ minimum. Extend $f$ to $v_{1}$ from $x$.

- $B_{1}$ is trivial and $B_{2}$ is 2 -connected.

- If $v_{1}$ has no neighbor in $V\left(\bar{G}_{i}\right)$, extend $f$ to $v_{1}$ from $u_{2}$.

- If $v_{1}$ has a neighbor in $V\left(\bar{G}_{i}\right)$. Let $x \in N_{G}\left(v_{1}\right) \cap V\left(\bar{G}_{i}\right)$ with $f(x)$ minimum. If $f(x)>f\left(u_{2}\right)$ then extend $f$ to $v_{1}$ from $u_{2}$. If $f(x) \leq f\left(u_{2}\right)$ then extend $f$ to $v_{1}$ from $x$.

Extend (the resulting) $f$ to $N_{B_{k}}\left(v_{k}\right)$ according to the following cases.

- $B_{k}$ is 2-connected. Let $\sigma$ denote a $\left(T_{u_{k}}^{k}, T_{w_{k}}^{k}\right)$-ordering of $N_{B_{k}}\left(v_{k}\right)=N_{B_{k}^{+}}\left(v_{k}\right)-\left\{u_{k}, w_{k}\right\}$ (the existence of this ordering is guaranteed by (3.4)). Extend $f$ to $\sigma$ from $u_{k}$.

- Both $B_{k}$ and $B_{k-1}$ are trivial. Let $x \in N_{G}\left(v_{k-1}\right) \cap V\left(\bar{G}_{i}\right)$ with $f(x)$ minimum. Extend $f$ to $v_{k-1}$ from $x$.

- $B_{k}$ is trivial and $B_{k-1}$ is 2 -connected.

- If $v_{k-1}$ has no neighbor in $V\left(\bar{G}_{i}\right)$, extend $f$ to $v_{k-1}$ from $u_{k-1}$.

- If $v_{k-1}$ has a neighbor in $V\left(\bar{G}_{i}\right)$, let $x \in N_{G}\left(v_{k-1}\right) \cap V\left(\bar{G}_{i}\right)$ with $f(x)$ minimum. If $f(x)>f\left(u_{k-1}\right)$ then extend $f$ to $v_{k-1}$ from $u_{k-1}$. If $f(x) \leq f\left(u_{k-1}\right)$ then extend $f$ to $v_{k-1}$ from $x$.

Case 4: $H_{i}$ is a triangle $G_{i-1}$-chain in $G$.

Let $I\left(H_{i}\right):=\left\{v_{1}, v_{2}, v_{3}\right\}$, let $v_{j} x_{j}(j=1,2,3)$ be the legs of $H_{i}$, and let $y_{1}, y_{2}, y_{3} \in V\left(\bar{G}_{i}\right)$ such that $y_{1} v_{1}, y_{2} v_{2}, y_{3} v_{3} \in E(G)$. Assume that $v_{1}, v_{2}, v_{3}$ are labeled so that $g\left(x_{1}\right)<g\left(x_{2}\right)<g\left(x_{3}\right)$. Let $D_{i}^{\prime}:=D_{i+1}^{\prime} \cup\left\{v_{1}, v_{2}, v_{3}\right\}$.

- If $f\left(y_{1}\right)<f\left(y_{2}\right)$ and $f\left(y_{1}\right)<f\left(y_{3}\right)$, then extend $f$ to $v_{1}, v_{2}, v_{3}$ from $y_{1}$.

- If $f\left(y_{2}\right)<f\left(y_{1}\right)$ and $f\left(y_{2}\right)<f\left(y_{3}\right)$, then extend $f$ to $v_{2}, v_{1}, v_{3}$ from $y_{2}$. 
- If $f\left(y_{3}\right)<f\left(y_{1}\right)<f\left(y_{2}\right)$, then extend $f$ to $v_{3}$ from $y_{3}$, and extend (the resulting) $f$ to $v_{1}, v_{2}$ from $y_{1}$.

- If $f\left(y_{3}\right)<f\left(y_{2}\right)<f\left(y_{1}\right)$ then extend $f$ to $v_{3}$ from $y_{3}$, and extend (the resulting) $f$ to $v_{2}, v_{1}$ from $y_{2}$.

This concludes the description of the algorithm for computing $f$. The proof of the next lemma is similar to the proof of (4.6) and we omit it.

(4.7) Lemma. Algorithm Numbering $f$ runs in $O\left(|V(G)|^{3}\right)$ time.

\section{Construction of spanning trees}

We describe now how to use Theorem (3.2) and the two numberings of the last section to produce four independent spanning trees. This will follow from Algorithm Trees below. The proof of its correction and analysis of its complexity will be given in the next section.

\section{Algorithm Trees.}

Description: Let $G$ be a 4-connected graph, $r \in V(G)$, and let $\mathcal{C}=\left(H_{1}, \ldots, H_{t}\right)$ be a nonseparating chain decomposition of $G$ rooted at $r$. Let $G_{0}=\bar{G}_{t}=(\{r\}, \emptyset)$, and for $1 \leq i \leq t-1$, let $G_{i}:=G\left[\bigcup_{j=1}^{i} I\left(H_{j}\right)\right]$ and $\bar{G}_{i}=G-\left(V\left(G_{i}\right)-\{r\}\right)$. The algorithm executes $t$ iterations, where $t$ is the number of chains in $\mathcal{C}$. At the first iteration we have $i=1$ and $T_{1}=T_{2}=T_{3}=T_{4}=G_{0}$. At the beginning of each iteration we have an integer $i$ with $1 \leq i \leq t$, spanning trees $T_{1}, T_{2}$ in $G_{i-1}$, and spanning forests $T_{3}, T_{4}$ in $G_{i-1}-r$.

Each iteration consists of the following: update $T_{1}, T_{2}, T_{3}, T_{4}$ by adding certain vertices and edges of $H_{i}$ to $T_{1}, T_{2}, T_{3}, T_{4}$ according to the following four cases (depending on the type of $H_{i}$ ), and, if $i<t$, then set $i \leftarrow i+1$ and start a new iteration. After $t$ iterations, $T_{1}, T_{2}, T_{3}, T_{4}$ will be independent spanning trees in $G$ rooted at $r$.

Case 1: $H_{i}$ is an elementary $G_{i-1}$-chain in $G$.

Let $H_{i}:=v_{0} B_{1} v_{1} B_{2} v_{2}$ with $g\left(v_{0}\right)<g\left(v_{2}\right)$. Let $v_{0}^{\prime}, v_{2}^{\prime}$ be neighbors of $v_{1}$ in $V\left(\bar{G}_{i}\right)$ with $f\left(v_{0}^{\prime}\right)<f\left(v_{2}^{\prime}\right)$.

Set $T_{1} \leftarrow T_{1}+\left\{v_{1}, v_{0} v_{1}\right\}, T_{2} \leftarrow T_{2}+\left\{v_{1}, v_{1} v_{2}\right\}, T_{3} \leftarrow T_{3}+\left\{v_{0}^{\prime}, v_{1}, v_{0}^{\prime} v_{1}\right\}$, and $T_{4} \leftarrow T_{4}+$ $\left\{v_{2}^{\prime}, v_{1}, v_{2}^{\prime} v_{1}\right\}$.

Case 2: $i=1$ or $H_{i}$ is an up $G_{i-1}$-chain in $G$, but not an elementary $G_{i-1}$-chain in $G$.

Let $H_{i}:=v_{0} B_{1} v_{1} \ldots v_{k-1} B_{k} v_{k}$, with $v_{0}=v_{k}=r$ when $i=1$, and $g\left(v_{0}\right)<g\left(v_{k}\right)$ when $i \neq 1$.

For each 2-connected block $B_{j}$, let $u_{j}, w_{j}$ denote the terminals of $B_{j}^{+}$other than $v_{j-1}, v_{j}$ with $f\left(u_{j}\right)<f\left(w_{j}\right)$, and let $T_{v_{j-1}}^{j}, T_{v_{j}}^{j}, T_{u_{j}}^{j}, T_{w_{j}}^{j}$ denote the trees rooted respectively at $v_{j-1}, v_{j}, u_{j}, w_{j}$ in the independent spanning $\left\{v_{j-1}, v_{j}, u_{j}, w_{j}\right\}$-system of $B_{j}^{+}$computed in (4.5).

Let $J:=\left\{j: 1 \leq j \leq k, B_{j}\right.$ is 2 -connected $\}$, and let $\bar{J}:=\{1, \ldots, k\}-J$.

First, set

$$
\begin{aligned}
& T_{1} \leftarrow T_{1} \cup\left(\bigcup_{j \in \bar{J}-\{k\}} B_{j}\right) \cup\left(\bigcup_{j \in J} T_{v_{j-1}}^{j}\right), \\
& T_{2} \leftarrow T_{2} \cup\left(\bigcup_{j \in \bar{J}-\{1\}} B_{j}\right) \cup\left(\bigcup_{j \in J} T_{v_{j}}^{j}\right), \\
& T_{3} \leftarrow T_{3} \cup\left(\bigcup_{j \in J} T_{u_{j}}^{j}\right), \text { and } \\
& T_{4} \leftarrow T_{4} \cup\left(\bigcup_{j \in J} T_{w_{j}}^{j}\right) .
\end{aligned}
$$


Now for each $j=1, \ldots, k-1$ add $v_{j}$ and edges incident to $v_{j}$ to $T_{1}, T_{2}, T_{3}, T_{4}$ according to the following cases. (At this stage, $v_{0}, v_{k} \notin V\left(T_{3} \cup T_{4}\right)$.)

Subcase 2.1: $B_{j}$ and $B_{j+1}$ are trivial.

Let $p_{3}, p_{4}$ be neighbors of $v_{j}$ in $V\left(\bar{G}_{i}\right)$ with $f\left(p_{3}\right)$ minimum (hence $f\left(p_{3}\right)<f\left(p_{4}\right)$ ).

Set $T_{3} \leftarrow T_{3}+\left\{v_{j}, p_{3}, v_{j} p_{3}\right\}$ and $T_{4} \leftarrow T_{4}+\left\{v_{j}, p_{4}, v_{j} p_{4}\right\}$.

Subcase 2.2: $B_{j}$ is 2-connected, and $B_{j+1}$ is trivial.

- If $v_{j}$ has no neighbor in $V\left(\bar{G}_{i}\right)$, then let $p_{1}, p_{3}, p_{4}$ be neighbors of $v_{j}$ in $B_{j}$ (they exist since $G$ is 4-connected) and assume that $p_{3}, p_{1}, p_{4}$ is $\left(T_{u_{j}}^{j}, T_{w_{j}}^{j}\right.$ )-ordered (this is possible by (3.4)). By Lemma (3.7), $T_{u_{j}}^{j}\left[u_{j}, p_{3}\right], T_{v_{j-1}}^{j}\left[v_{j-1}, p_{1}\right]$ and $T_{w_{j}}^{j}\left[w_{j}, p_{4}\right]$ are disjoint. If $k=2$, then we also require that $p_{3}, p_{1}, p_{4}$ be the vertices $q_{1}, q_{2}, q_{3}$, respectively, chosen in Subcase 3.2 of Algorithm Numbering $f$.

Set $T_{1} \leftarrow T_{1}+\left\{v_{j}, v_{j} p_{1}\right\}, T_{3} \leftarrow T_{3}+\left\{v_{j}, v_{j} p_{3}\right\}$, and $T_{4} \leftarrow T_{4}+\left\{v_{j}, v_{j} p_{4}\right\}$.

- If $v_{j}$ has a neighbor in $V\left(\bar{G}_{i}\right)$, then let $x \in N_{G}\left(v_{j}\right) \cap V\left(\bar{G}_{i}\right)$ with $f(x)$ minimum.

- If $f(x)>f\left(u_{j}\right)$ then let $p_{1}, p_{3}$ be neighbors of $v_{j}$ in $B_{j}$ such that the paths $T_{v_{j-1}}^{j}\left[v_{j-1}, p_{1}\right], T_{u_{j}}^{j}\left[u_{j}, p_{3}\right]$ are disjoint (they exist by (3.6)).

Set $T_{1} \leftarrow T_{1}+\left\{v_{j}, v_{j} p_{1}\right\}, T_{3} \leftarrow T_{3}+\left\{v_{j}, v_{j} p_{3}\right\}$, and $T_{4} \leftarrow T_{4}+\left\{v_{j}, x, v_{j} x\right\}$.

- If $f(x) \leq f\left(u_{j}\right)$ then let $p_{1}, p_{4}$ be neighbors of $v_{j}$ in $B_{j}$ such that the paths $T_{v_{j-1}}^{j}\left[v_{j-1}, p_{1}\right], T_{w_{j}}^{j}\left[w_{j}, p_{4}\right]$ are disjoint (they exist by (3.6)).

Set $T_{1} \leftarrow T_{1}+\left\{v_{j}, v_{j} p_{1}\right\}, T_{3} \leftarrow T_{3}+\left\{v_{j}, x, v_{j} x\right\}$, and $T_{4} \leftarrow T_{4}+\left\{v_{j}, v_{j} p_{4}\right\}$.

Subcase 2.3: $B_{j}$ is trivial, and $B_{j+1}$ is 2-connected.

- If $v_{j}$ has no neighbor in $V\left(\bar{G}_{i}\right)$, then let $p_{2}, p_{3}, p_{4}$ be neighbors of $v_{j}$ in $B_{j+1}$ (they exist since $G$ is 4-connected) and assume that $p_{3}, p_{2}, p_{4}$ is $\left(T_{u_{j+1}}^{j+1}, T_{w_{j+1}}^{j+1}\right)$-ordered (this is possible by (3.4)). By Lemma (3.7), $T_{u_{j+1}}^{j+1}\left[u_{j+1}, p_{3}\right], T_{v_{j+1}}^{j+1}\left[v_{j+1}, p_{2}\right] T_{w_{j+1}}^{j}\left[w_{j+1}, p_{4}\right]$ are disjoint. If $k=2$, then we also require that $p_{3}, p_{2}, p_{4}$ be the vertices $q_{1}, q_{2}, q_{3}$, respectively, chosen in Subcase 3.2. of Algorithm Numbering $f$.

Set $T_{2} \leftarrow T_{2}+\left\{v_{j}, v_{j} p_{2}\right\}, T_{3} \leftarrow T_{3}+\left\{v_{j}, v_{j} p_{3}\right\}$, and $T_{4} \leftarrow T_{4}+\left\{v_{j}, v_{j} p_{4}\right\}$.

- If $v_{j}$ has a neighbor in $V\left(\bar{G}_{i}\right)$, then let $x \in N_{G}\left(v_{j}\right) \cap V\left(\bar{G}_{i}\right)$ with $f(x)$ minimum.

- If $f(x)>f\left(u_{j+1}\right)$ then let $p_{2}, p_{3}$ be neighbors of $v_{j}$ in $B_{j+1}$ such that the paths $T_{v_{j+1}}^{j+1}\left[v_{j+1}, p_{2}\right], T_{u_{j+1}}^{j+1}\left[u_{j+1}, p_{3}\right]$ are disjoint (they exist by (3.6)).

Set $T_{2} \leftarrow T_{2}+\left\{v_{j}, v_{j} p_{2}\right\}, T_{3} \leftarrow T_{3}+\left\{v_{j}, v_{j} p_{3}\right\}$, and $T_{4} \leftarrow T_{4}+\left\{v_{j}, x, v_{j} x\right\}$.

- If $f(x) \leq f\left(u_{j+1}\right)$ then let $p_{2}, p_{4}$ be neighbors of $v_{j}$ in $B_{j+1}$ such that the paths $T_{v_{j+1}}^{j+1}\left[v_{j+1}, p_{2}\right], T_{w_{j+1}}^{j+1}\left[w_{j+1}, p_{4}\right]$ are disjoint (they exist by $\left.(3.6)\right)$.

Set $T_{2} \leftarrow T_{2}+\left\{v_{j}, v_{j} p_{2}\right\}, T_{3} \leftarrow T_{3}+\left\{v_{j}, x, v_{j} x\right\}$, and $T_{4} \leftarrow T_{4}+\left\{v_{j}, v_{j} p_{4}\right\}$.

Subcase 2.4: $B_{j}$ and $B_{j+1}$ are 2-connected.

Note that $f\left(u_{j}\right)<f\left(w_{j+1}\right)$ or $f\left(u_{j+1}\right)<f\left(w_{j}\right)$.

- If $f\left(u_{j}\right)<f\left(w_{j+1}\right)$, then let $p_{1}, p_{3}$ be neighbors of $v_{j}$ in $B_{j}$ such that the paths $T_{v_{j-1}}^{j}\left[v_{j-1}, p_{1}\right], T_{u_{j}}^{j}\left[u_{j}, p_{3}\right]$ are disjoint (they exist by (3.6)), and let $p_{2}, p_{4}$ be neighbors of $v_{j}$ in $B_{j+1}$ such that the paths $T_{v_{j+1}}^{j+1}\left[v_{j+1}, p_{2}\right], T_{w_{j+1}}^{j+1}\left[w_{j+1}, p_{4}\right]$ are disjoint (they exist by (3.6)). Set $T_{1} \leftarrow T_{1}+\left\{v_{j}, v_{j} p_{1}\right\}, T_{2} \leftarrow T_{2}+\left\{v_{j}, v_{j} p_{2}\right\}, T_{3} \leftarrow T_{3}+\left\{v_{j}, v_{j} p_{3}\right\}$, and $T_{4} \leftarrow T_{4}+$ $\left\{v_{j}, v_{j} p_{4}\right\}$. 
- If $f\left(u_{j}\right) \geq f\left(w_{j+1}\right)$ then $f\left(u_{j+1}\right)<f\left(w_{j}\right)$. Let $p_{1}, p_{4}$ be neighbors of $v_{j}$ in $B_{j}$ such that the paths $T_{v_{j-1}}^{j}\left[v_{j-1}, p_{1}\right], T_{w_{j}}^{j}\left[w_{j}, p_{4}\right]$ are disjoint (they exist by (3.6)), and let $p_{2}, p_{3}$ be neighbors of $v_{j}$ in $B_{j+1}$ such that the paths $T_{v_{j+1}}^{j+1}\left[v_{j+1}, p_{2}\right], T_{u_{j+1}}^{j+1}\left[u_{j+1}, p_{3}\right]$ are disjoint (they exist by (3.6)).

Set $T_{1} \leftarrow T_{1}+\left\{v_{j}, v_{j} p_{1}\right\}, T_{2} \leftarrow T_{2}+\left\{v_{j}, v_{j} p_{2}\right\}, T_{3} \leftarrow T_{3}+\left\{v_{j}, v_{j} p_{3}\right\}$, and $T_{4} \leftarrow T_{4}+$ $\left\{v_{j}, v_{j} p_{4}\right\}$.

Case 3: $i=t$ or $H_{i}$ is a down $G_{i-1}$-chain in $G$.

Let $H_{i}:=v_{0} B_{1} v_{1} \ldots v_{k-1} B_{k} v_{k}$, with $v_{0}=v_{k}=r$ when $i=t$, and $f\left(v_{0}\right)<f\left(v_{k}\right)$ when $i \neq t$.

For each 2-connected block $B_{j}$, let $u_{j}, w_{j}$ denote the terminals of $B_{j}^{+}$other than $v_{j-1}, v_{j}$, with $g\left(u_{j}\right)<g\left(w_{j}\right)$, and let $T_{v_{j-1}}^{j}, T_{v_{j}}^{j}, T_{u_{j}}^{j}, T_{w_{j}}^{j}$ denote the trees rooted respectively at $v_{j-1}, v_{j}, u_{j}, w_{j}$ in the independent spanning $\left\{v_{j-1}, v_{j}, u_{j}, w_{j}\right\}$-system of $B_{j}^{+}$computed in (4.5).

Let $J:=\left\{j: 1 \leq j \leq k, B_{j}\right.$ is 2-connected $\}$, and let $\bar{J}:=\{1, \ldots, k\}-J$.

First, set

$$
\begin{aligned}
T_{1} & \leftarrow T_{1} \cup\left(\bigcup_{j \in J} T_{u_{j}}^{j}\right), \\
T_{2} & \leftarrow T_{2} \cup\left(\bigcup_{j \in J} T_{w_{j}}^{j}\right), \\
T_{3} & \leftarrow T_{3} \cup\left(\bigcup_{j \in \bar{J}-\{k\}} B_{j}\right) \cup\left(\bigcup_{j \in J} T_{v_{j-1}}^{j}\right), \text { and } \\
T_{4} & \leftarrow T_{4} \cup\left(\bigcup_{j \in \bar{J}-\{1\}} B_{j}\right) \cup\left(\bigcup_{j \in J} T_{v_{j}}^{j}\right) .
\end{aligned}
$$

Now for each $j=1, \ldots, k-1$ add $v_{j}$ and edges incident to $v_{j}$ to $T_{1}, T_{2}, T_{3}, T_{4}$ according to the following cases. (At this stage $v_{0}, v_{k} \notin V\left(T_{1} \cup T_{2}\right)$.

Subcase 3.1: $B_{j}$ and $B_{j+1}$ are trivial blocks.

Let $p_{1}, p_{2}$ be neighbors of $v_{j}$ in $V\left(G_{i-1}\right)$ with $g\left(p_{1}\right)$ minimum (hence $g\left(p_{1}\right)<g\left(p_{2}\right)$ ).

Set $T_{1} \leftarrow T_{1}+\left\{v_{j}, v_{j} p_{1}\right\}$ and $T_{2} \leftarrow T_{2}+\left\{v_{j}, v_{j} p_{2}\right\}$.

Subcase 3.2: $B_{j}$ is 2-connected, and $B_{j+1}$ is trivial.

- If $v_{j}$ has no neighbor in $V\left(G_{i-1}\right)$, then let $p_{1}, p_{2}, p_{3}$ be neighbors of $v_{j}$ in $B_{j}$ (they exist since $G$ is 4-connected) and assume that $p_{1}, p_{3}, p_{2}$ is $\left(T_{u_{j}}^{j}, T_{w_{j}}^{j}\right)$-ordered (this is possible by (3.4)). By Lemma (3.7), $T_{u_{j}}^{j}\left[u_{j}, p_{1}\right], T_{v_{j-1}}^{j}\left[v_{j-1}, p_{3}\right]$ and $T_{w_{j}}^{j}\left[w_{j}, p_{2}\right]$ are disjoint. If $k=2$ then we also require that $p_{1}, p_{3}, p_{2}$ be the vertices $q_{1}, q_{2}, q_{3}$, respectively, chosen in Subcase 3.2 of Algorithm Numbering $g$.

Set $T_{1} \leftarrow T_{1}+\left\{v_{j}, v_{j} p_{1}\right\}, T_{2} \leftarrow T_{2}+\left\{v_{j}, v_{j} p_{2}\right\}$, and $T_{3} \leftarrow T_{3}+\left\{v_{j}, v_{j} p_{3}\right\}$.

- If $v_{j}$ has a neighbor in $V\left(G_{i-1}\right)$, then let $x \in N_{G}\left(v_{j}\right) \cap V\left(G_{i-1}\right)$ with $g(x)$ minimum.

- If $g(x)>g\left(u_{j}\right)$ then let $p_{1}, p_{3}$ be neighbors of $v_{j}$ in $B_{j}$ such that the paths $T_{u_{j}}^{j}\left[u_{j}, p_{1}\right]$ and $T_{v_{j-1}}^{j}\left[v_{j-1}, p_{3}\right]$ are disjoint (they exist by $\left.(3.6)\right)$.

Set $T_{1} \leftarrow T_{1}+\left\{v_{j}, v_{j} p_{1}\right\}, T_{2} \leftarrow T_{2}+\left\{v_{j}, v_{j} x\right\}$, and $T_{3} \leftarrow T_{3}+\left\{v_{j}, v_{j} p_{3}\right\}$.

- If $g(x) \leq g\left(u_{j}\right)$ then let $p_{2}, p_{3}$ be neighbors of $v_{j}$ in $B_{j}$ such that the paths $T_{w_{j}}^{j}\left[w_{j}, p_{2}\right]$ and $T_{v_{j-1}}^{j}\left[v_{j-1}, p_{3}\right]$ are disjoint (they exist by (3.6)).

Set $T_{1} \leftarrow T_{1}+\left\{v_{j}, v_{j} x\right\}, T_{2} \leftarrow T_{2}+\left\{v_{j}, v_{j} p_{2}\right\}$, and $T_{3} \leftarrow T_{3}+\left\{v_{j}, v_{j} p_{3}\right\}$.

Subcase 3.3: $B_{j}$ is trivial, and $B_{j+1}$ is 2 -connected. 
- If $v_{j}$ has no neighbor in $V\left(G_{i-1}\right)$, then let $p_{1}, p_{2}, p_{4}$ be neighbors of $v_{j}$ in $B_{j+1}$ (they exist since $G$ is 4-connected) and assume that $p_{1}, p_{4}, p_{2}$ is $\left(T_{u_{j+1}}^{j+1}, T_{w_{j+1}}^{j+1}\right)$-ordered (this is possible by (3.4)). By Lemma (3.7), $T_{u_{j+1}}^{j+1}\left[u_{j+1}, p_{1}\right], T_{v_{j+1}}^{j+1}\left[v_{j+1}, p_{4}\right]$ and $T_{w_{j+1}}^{j+1}\left[w_{j+1}, p_{2}\right]$ are disjoint. If $k=2$, then we also require that $p_{1}, p_{4}, p_{2}$ be the vertices $q_{1}, q_{2}, q_{3}$, respectively, chosen in Subcase 3.2 of Algorithm Numbering $g$.

Set $T_{1} \leftarrow T_{1}+\left\{v_{j}, v_{j} p_{1}\right\}, T_{2} \leftarrow T_{2}+\left\{v_{j}, v_{j} p_{2}\right\}$, and $T_{4} \leftarrow T_{4}+\left\{v_{j}, v_{j} p_{4}\right\}$.

- If $v_{j}$ has a neighbor in $V\left(G_{i-1}\right)$, then let $x \in N_{G}\left(v_{j}\right) \cap V\left(G_{i-1}\right)$ with $g(x)$ minimum.

- If $g(x)>g\left(u_{j+1}\right)$ then let $p_{1}, p_{4}$ be neighbors of $v_{j}$ in $B_{j+1}$ such that the paths $T_{u_{j+1}}^{j+1}\left[u_{j+1}, p_{1}\right], T_{v_{j+1}}^{j+1}\left[v_{j+1}, p_{4}\right]$ are disjoint (they exist by (3.6)).

Set $T_{1} \leftarrow T_{1}+\left\{v_{j}, v_{j} p_{1}\right\}, T_{2} \leftarrow T_{2}+\left\{v_{j}, v_{j} x\right\}$, and $T_{4} \leftarrow T_{4}+\left\{v_{j}, v_{j} p_{4}\right\}$.

- If $g(x) \leq g\left(u_{j+1}\right)$ let $p_{2}, p_{4}$ be neighbors of $v_{j}$ in $B_{j+1}$ such that the paths $T_{w_{j+1}}^{j+1}\left[w_{j+1}, p_{2}\right], T_{v_{j+1}}^{j+1}\left[v_{j+1}, p_{4}\right]$ are disjoint (they exist by $\left.(3.6)\right)$.

Set $T_{1} \leftarrow T_{1}+\left\{v_{j}, v_{j} x\right\}, T_{2} \leftarrow T_{2}+\left\{v_{j}, v_{j} p_{2}\right\}$, and $T_{4} \leftarrow T_{4}+\left\{v_{j}, v_{j} p_{4}\right\}$.

Subcase 3.4: $B_{j}$ and $B_{j+1}$ are 2-connected.

Note that $g\left(u_{j}\right)<g\left(w_{j+1}\right)$ or $g\left(u_{j+1}\right)<g\left(w_{j}\right)$.

- If $g\left(u_{j}\right)<g\left(w_{j+1}\right)$ then let $p_{1}, p_{3}$ be neighbors of $v_{j}$ in $B_{j}$ such that the paths $T_{u_{j}}^{j}\left[u_{j}, p_{1}\right], T_{v_{j-1}}^{j}\left[v_{j-1}, p_{3}\right]$ are disjoint (they exist by (3.6)), and let $p_{2}, p_{4}$ be neighbors of $v_{j}$ in $B_{j+1}$ such that the paths $T_{w_{j+1}}^{j+1}\left[w_{j+1}, p_{2}\right]$ and $T_{v_{j+1}}^{j+1}\left[v_{j+1}, p_{4}\right]$ are disjoint (they exist by $(3.6))$.

Set $T_{1} \leftarrow T_{1}+\left\{v_{j}, v_{j} p_{1}\right\}, T_{2} \leftarrow T_{2}+\left\{v_{j}, v_{j} p_{2}\right\}, T_{3} \leftarrow T_{3}+\left\{v_{j}, v_{j} p_{3}\right\}$, and $T_{4} \leftarrow T_{4}+$ $\left\{v_{j}, v_{j} p_{4}\right\}$.

- If $g\left(u_{j}\right) \geq g\left(w_{j+1}\right)$ then $g\left(u_{j+1}\right)<g\left(w_{j}\right)$. Let $p_{2}, p_{3}$ be neighbors of $v_{j}$ in $B_{j}$ such that the paths $T_{w_{j}}^{j}\left[w_{j}, p_{2}\right]$ and $T_{v_{j-1}}^{j}\left[v_{j-1}, p_{3}\right]$ are disjoint (they exist by (3.6)), and let $p_{1}, p_{4}$ be neighbors of $v_{j}$ in $B_{j+1}$ such that the paths $T_{u_{j+1}}^{j+1}\left[u_{j+1}, p_{1}\right]$ and $T_{v_{j+1}}^{j+1}\left[v_{j+1}, p_{4}\right]$ are disjoint (they exist by (3.6)).

Set $T_{1} \leftarrow T_{1}+\left\{v_{j}, v_{j} p_{1}\right\}, T_{2} \leftarrow T_{2}+\left\{v_{j}, v_{j} p_{2}\right\}, T_{3} \leftarrow T_{3}+\left\{v_{j}, v_{j} p_{3}\right\}$, and $T_{4} \leftarrow T_{4}+$ $\left\{v_{j}, v_{j} p_{4}\right\}$.

Case 4: $H_{i}$ is a triangle $G_{i-1}$-chain in $G$.

Let $I\left(H_{i}\right):=\left\{v_{1}, v_{2}, v_{3}\right\}$, let $v_{j} x_{j}(j=1,2,3)$ be the legs of $H_{i}$, and let $y_{1}, y_{2}, y_{3} \in V\left(\bar{G}_{i}\right)$ such that $y_{1} v_{1}, y_{2} v_{2}, y_{3} v_{3} \in E(G)$. Assume that $v_{1}, v_{2}, v_{3}$ are labeled so that $g\left(x_{1}\right)<g\left(x_{2}\right)<g\left(x_{3}\right)$.

Update $T_{1}, T_{2}, T_{3}, T_{4}$ according to the following four possibilities.

- $f\left(y_{1}\right)<f\left(y_{2}\right)$ and $f\left(y_{1}\right)<f\left(y_{3}\right)$.

Set

$T_{1} \leftarrow T_{1}+\left\{v_{1}, v_{2}, v_{3}, x_{1} v_{1}, x_{2} v_{2}, v_{2} v_{3}\right\}, T_{2} \leftarrow T_{2}+\left\{v_{1}, v_{2}, v_{3}, x_{3} v_{3}, v_{3} v_{1}, v_{3} v_{2}\right\}, T_{3} \leftarrow T_{3}+$ $\left\{v_{1}, v_{2}, v_{3}, y_{1} v_{1}, v_{1} v_{2}, v_{1} v_{3}\right\}$, and $T_{4} \leftarrow T_{4}+\left\{v_{1}, v_{2}, v_{3}, y_{2} v_{2}, v_{2} v_{1}, y_{3} v_{3}\right\}$.

- $f\left(y_{2}\right)<f\left(y_{1}\right)$ and $f\left(y_{2}\right)<f\left(y_{3}\right)$.

Set

$T_{1} \leftarrow T_{1}+\left\{v_{1}, v_{2}, v_{3}, x_{1} v_{1}, x_{2} v_{2}, v_{1} v_{3}\right\}, T_{2} \leftarrow T_{2}+\left\{v_{1}, v_{2}, v_{3}, x_{3} v_{3}, v_{3} v_{1}, v_{3} v_{2}\right\}, T_{3} \leftarrow T_{3}+$ $\left\{v_{1}, v_{2}, v_{3}, y_{2} v_{2}, v_{2} v_{1}, v_{2} v_{3}\right\}$, and $T_{4} \leftarrow T_{4}+\left\{v_{1}, v_{2}, v_{3}, y_{1} v_{1}, v_{1} v_{2}, y_{3} v_{3}\right\}$.

- $f\left(y_{3}\right)<f\left(y_{1}\right)<f\left(y_{2}\right)$.

Set

$T_{1} \leftarrow T_{1}+\left\{v_{1}, v_{2}, v_{3}, x_{1} v_{1}, x_{2} v_{2}, v_{1} v_{3}\right\}, T_{2} \leftarrow T_{2}+\left\{v_{1}, v_{2}, v_{3}, x_{3} v_{3}, v_{3} v_{1}, v_{3} v_{2}\right\}, T_{3} \leftarrow T_{3}+$ $\left\{v_{1}, v_{2}, v_{3}, y_{1} v_{1}, v_{1} v_{2}, y_{3} v_{3}\right\}$, and $T_{4} \leftarrow T_{4}+\left\{v_{1}, v_{2}, v_{3}, y_{2} v_{2}, v_{2} v_{1}, v_{2} v_{3}\right\}$. 
- $f\left(y_{3}\right)<f\left(y_{2}\right)<f\left(y_{1}\right)$.

Set

$T_{1} \leftarrow T_{1}+\left\{v_{1}, v_{2}, v_{3}, x_{1} v_{1}, x_{2} v_{2}, v_{2} v_{3}\right\}, T_{2} \leftarrow T_{2}+\left\{v_{1}, v_{2}, v_{3}, x_{3} v_{3}, v_{3} v_{1}, v_{3} v_{2}\right\}, T_{3} \leftarrow T_{3}+$ $\left\{v_{1}, v_{2}, v_{3}, y_{2} v_{2}, v_{2} v_{1}, y_{3} v_{3}\right\}$, and $T_{4} \leftarrow T_{4}+\left\{v_{1}, v_{2}, v_{3}, y_{1} v_{1}, v_{1} v_{2}, v_{1} v_{3}\right\}$.

\section{Correctness of Algorithm Trees}

In this section we will prove Theorem (1.1). More precisely, we will show that the subgraphs $T_{1}, T_{2}, T_{3}, T_{4}$ returned by Algorithm Trees in the previous section are independent spanning trees of $G$ rooted at $r$, and they can be computed in $O\left(|V(G)|^{3}\right)$ time.

(6.1) Notation. Let $G$ be a 4 -connected graph, $r \in V(G)$, and let $\mathcal{C}=\left(H_{1}, \ldots, H_{t}\right)$ be a nonseparating chain decomposition of $G$ rooted at $r$. Let $G_{0}=\bar{G}_{t}=(\{r\}, \emptyset)$, and for $1 \leq i \leq t-1$, let $G_{i}:=G\left[\bigcup_{j=1}^{i} I\left(H_{j}\right)\right]$ and $\bar{G}_{i}=G-\left(V\left(G_{i}\right)-\{r\}\right)$. Let $T_{1}, T_{2}, T_{3}, T_{4}$ denote the subgraphs returned by Algorithm Trees. Let $D, D^{\prime}$ denote the sets of vertices returned by Algorithm Numbering $g$ and Algorithm Numbering $f$, respectively.

We start with a series of seven simple lemmas which follows from the cases of Algorithm Trees. The first lemma follows immediately by inspecting Case 1 of Algorithm Trees.

(6.2) Lemma. Let $H_{i}:=v_{0} B_{1} v_{1} B_{2} v_{2}$ be an elementary $G_{i-1}$-chain in $G$, with $g\left(v_{0}\right)<g\left(v_{2}\right)$. Then $v_{1}$ has neighbors $v_{0}^{\prime}, v_{2}^{\prime}$ in $V\left(\bar{G}_{i}\right)$, with $f\left(v_{0}^{\prime}\right)<f\left(v_{2}^{\prime}\right)$, such that

(1) $E\left(T_{1} \cap H_{i}\right)=\left\{v_{0} v_{1}\right\}$ and $E\left(T_{2} \cap H_{i}\right)=\left\{v_{1} v_{2}\right\}$, and

(2) $E\left(T_{3} \cap H_{i}^{\prime}\right)=\left\{v_{0}^{\prime} v_{1}\right\}$ and $E\left(T_{4} \cap H_{i}^{\prime}\right)=\left\{v_{1} v_{2}^{\prime}\right\}$, where $H_{i}^{\prime}=v_{0}^{\prime} B_{1}^{\prime} v_{1} B_{2}^{\prime} v_{2}^{\prime}$ is an elementary $\bar{G}_{i}$-chain in $G$.

The next lemma follows by inspecting Case $2($ for $i=1$ ) of Algorithm Trees.

(6.3) Lemma. Let $H_{1}:=v_{0} B_{1} v_{1} \ldots v_{k-1} B_{k} v_{k}$ with $v_{0}=v_{k}=r$, and for each 2-connected $B_{j}$, let $u_{j}, w_{j}$ denote the terminals of $B_{j}^{+}$other than $v_{j-1}, v_{j}$, with $f\left(u_{j}\right)<f\left(w_{j}\right)$. Let $H_{1}^{+}$be the graph obtained from $H_{1}$ by adding $N_{G}\left(H_{1}-r\right)-\{r\}$ and the edges of $G$ from $V\left(H_{1}\right)$ to $N_{G}\left(H_{1}-r\right)-\{r\}$. Then

(1) $T_{1} \cap H_{1}$ is a spanning tree of $H_{1}$ rooted at $r$ and contains no edge from $r$ to $N_{B_{k}}(r)$,

(2) $T_{2} \cap H_{1}$ is a spanning tree of $H_{1}$ rooted at $r$ and contains no edge from $r$ to $N_{B_{1}}(r)$,

(3) $T_{3} \cap\left(H_{1}^{+}-r\right)$ is a spanning forest of $H_{1}^{+}-r$, and each component of $T_{3} \cap\left(H_{1}^{+}-r\right)$ either is a tree in $B_{j}^{+}-w_{j}$ rooted at $u_{j}$ for some $j \in\{1, \ldots, k\}$, or is induced by a single edge with one end in $V\left(\bar{G}_{1}\right)$ and the other in $\left\{v_{1}, \ldots, v_{k-1}\right\}$, and

(4) $T_{4} \cap\left(H_{1}^{+}-r\right)$ is a spanning forest of $H_{1}^{+}-r$, and each component of $T_{4} \cap\left(H_{1}^{+}-r\right)$ either is a tree in $B_{j}^{+}-u_{j}$ rooted at $w_{j}$ for some $j \in\{1, \ldots, k\}$, or is induced by a single edge with one end in $V\left(\bar{G}_{1}\right)$ and the other in $\left\{v_{1}, \ldots, v_{k-1}\right\}$.

By inspecting Case 2 (for $i \neq 1$ ) of Algorithms Trees, we have

(6.4) Lemma. Let $H_{i}:=v_{0} B_{1} v_{1} \ldots v_{k-1} B_{k} v_{k}(2 \leq i \leq t-1)$ with $g\left(v_{0}\right)<g\left(v_{k}\right)$, and for each 2connected block $B_{j}$, let $u_{j}, w_{j}$ denote the terminals of $B_{j}^{+}$other than $v_{j-1}, v_{j}$, with $f\left(u_{j}\right)<f\left(w_{j}\right)$. Let $H_{i}^{+}$be the graph obtained from $H_{i}$ by adding $N_{G}\left(H_{i}-\left\{v_{0}, v_{k}\right\}\right)-\left\{v_{0}, v_{k}\right\}$ and the edges of $G$ from $V\left(H_{i}\right)$ to $N_{G}\left(H_{i}-\left\{v_{0}, v_{k}\right\}\right)-\left\{v_{0}, v_{k}\right\}$. Then

(1) $T_{1} \cap\left(H_{i}-v_{k}\right)$ is a spanning tree of $H_{i}-v_{k}$ rooted at $v_{0}$ and $T_{1}$ contains no edge from $v_{k}$ to $N_{B_{k}}\left(v_{k}\right)$, 
(2) $T_{2} \cap\left(H_{i}-v_{0}\right)$ is a spanning tree of $H_{i}-v_{0}$ rooted at $v_{k}$ and $T_{2}$ contains no edge from $v_{0}$ to $N_{B_{1}}\left(v_{0}\right)$,

(3) $T_{3} \cap\left(H_{i}^{+}-\left\{v_{0}, v_{k}\right\}\right)$ is a spanning forest of $H_{i}^{+}-\left\{v_{0}, v_{k}\right\}$, and each component of $T_{3} \cap\left(H_{i}^{+}-\right.$ $\left.\left\{v_{0}, v_{k}\right\}\right)$ either is a tree in $B_{j}^{+}-w_{j}$ rooted at $u_{j}$ for some $j \in\{1, \ldots, k\}$, or is induced by a single edge with one end in $V\left(\bar{G}_{i}\right)$ and the other in $\left\{v_{1}, \ldots, v_{k-1}\right\}$, and

(4) $T_{4} \cap\left(H_{i}^{+}-\left\{v_{0}, v_{k}\right\}\right)$ is a spanning forest of $H_{i}^{+}-\left\{v_{0}, v_{k}\right\}$, and each component of $T_{4} \cap\left(H_{i}^{+}-\right.$ $\left.\left\{v_{0}, v_{k}\right\}\right)$ either is a tree in $B_{j}^{+}-u_{j}$ rooted at $w_{j}$ for some $j \in\{1, \ldots, k\}$, or is induced by a single edge with one end in $V\left(\bar{G}_{i}\right)$ and the other in $\left\{v_{1}, \ldots, v_{k-1}\right\}$.

By a simple inspection of Case 3 (for $i=t$ ), we have

(6.5) Lemma. Let $H_{t}:=v_{0} B_{1} v_{1} \ldots v_{k-1} B_{k} v_{k}$ with $v_{0}=v_{k}=r$, and for each 2-connected $B_{j}$, let $u_{j}, w_{j}$ denote the terminals of $B_{j}^{+}$other than $v_{j-1}, v_{j}$, with $g\left(u_{j}\right)<g\left(w_{j}\right)$. Let $H_{t}^{+}$be the graph obtained from $H_{t}$ by adding $N_{G}\left(H_{t}-r\right)-\{r\}$ and the edges of $G$ from $V\left(H_{t}\right)$ to $N_{G}\left(H_{t}-r\right)-\{r\}$. Then

(1) $T_{1} \cap\left(H_{t}^{+}-r\right)$ is a spanning forest of $H_{t}^{+}-r$, and each component of $T_{1} \cap\left(H_{t}^{+}-r\right)$ either is a tree in $B_{j}^{+}-w_{j}$ rooted at $u_{j}$ for some $j \in\{1, \ldots, k\}$, or is induced by a single edge with one end in $V\left(G_{i-1}\right)$ and the other in $\left\{v_{1}, \ldots, v_{k-1}\right\}$,

(2) $T_{2} \cap\left(H_{t}^{+}-r\right)$ is a spanning forest of $H_{t}^{+}-r$, and each component of $T_{2} \cap\left(H_{t}^{+}-r\right)$ either is a tree in $B_{j}^{+}-u_{j}$ rooted at $w_{j}$ for some $j \in\{1, \ldots, k\}$, or is induced by a single edge with one end in $V\left(G_{i-1}\right)$ and the other in $\left\{v_{1}, \ldots, v_{k-1}\right\}$,

(3) $T_{3} \cap H_{t}$ is a spanning tree of $H_{t}$ rooted at $r$ and contains no edge from $r$ to $N_{B_{k}}(r)$, and

(4) $T_{4} \cap H_{t}$ is a spanning tree of $H_{t}$ rooted at $r$ and contains no edge from $r$ to $N_{B_{1}}(r)$.

The next lemma follows from a simple inspection of Case 3 (for $i \neq t$ ).

(6.6) Lemma. Let $H_{i}:=v_{0} B_{1} v_{1} \ldots v_{k-1} B_{k} v_{k}(2 \leq i \leq t-1)$ with $f\left(v_{0}\right)<f\left(v_{k}\right)$, and for each 2connected block $B_{j}$, let $u_{j}, w_{j}$ denote the terminals of $B_{j}^{+}$other than $v_{j-1}, v_{j}$, with $g\left(u_{j}\right)<g\left(w_{j}\right)$. Let $H_{i}^{+}$be the graph obtained from $H_{i}$ by adding $N_{G}\left(H_{i}-\left\{v_{0}, v_{k}\right\}\right)-\left\{v_{0}, v_{k}\right\}$ and the edges of $G$ from $V\left(H_{i}\right)$ to $N_{G}\left(H_{i}-\left\{v_{0}, v_{k}\right\}\right)-\left\{v_{0}, v_{k}\right\}$. Then

(1) $T_{1} \cap\left(H_{i}^{+}-\left\{v, v_{k}\right\}\right)$ is a spanning forest of $H_{i}^{+}-\left\{v_{0}, v_{k}\right\}$, and each component of $T_{1} \cap\left(H_{i}^{+}-\right.$ $\left.\left\{v_{0}, v_{k}\right\}\right)$ either is a tree in $B_{j}^{+}-w_{j}$ rooted at $u_{j}$ for some $j \in\{1, \ldots, k\}$, or is induced by a single edge with one end in $V\left(G_{i-1}\right)$ and the other in $\left\{v_{1}, \ldots, v_{k-1}\right\}$,

(2) $T_{2} \cap\left(H_{i}^{+}-\left\{v_{0}, v_{k}\right\}\right)$ is a spanning forest of $H_{i}^{+}-\left\{v_{0}, v_{k}\right\}$, and each component of $T_{2} \cap\left(H_{i}^{+}-\right.$ $\left.\left\{v_{0}, v_{k}\right\}\right)$ either is a tree in $B_{j}^{+}-u_{j}$ rooted at $w_{j}$ for some $j \in\{1, \ldots, k\}$, or is induced by a single edge with one end in $V\left(G_{i-1}\right)$ and the other in $\left\{v_{1}, \ldots, v_{k-1}\right\}$,

(3) $T_{3} \cap\left(H_{i}-v_{k}\right)$ is a spanning tree of $H_{i}-v_{k}$ rooted at $v_{0}$ and $T_{3}$ contains no edge from $v_{k}$ to $N_{B_{k}}\left(v_{k}\right)$, and

(4) $T_{4} \cap\left(H_{i}-v_{0}\right)$ is a spanning tree of $H_{i}-v_{0}$ rooted at $v_{k}$ and $T_{4}$ contains no edge from $v_{0}$ to $N_{B_{1}}\left(v_{0}\right)$.

Finally, by a simple inspection of Case 4 of Algorithm Trees, we have

(6.7) Lemma. Let $H_{i}$ be a triangle $G_{i-1}$-chain in $G(2 \leq i \leq t-1)$. Let $I\left(H_{i}\right):=\left\{v_{1}, v_{2}, v_{3}\right\}$, let $y_{1}, y_{2}, y_{3} \in V\left(\bar{G}_{i}\right)$ such that $y_{1} v_{1}, y_{2} v_{2}, y_{3} v_{3} \in E(G)$, and let $v_{j} x_{j}(j=1,2,3)$ be the legs of $H_{i}$, with $g\left(x_{1}\right)<g\left(x_{2}\right)<g\left(x_{3}\right)$. Let $H_{i}^{+}:=H_{i}+\left\{y_{1}, y_{2}, y_{3}, y_{1} v_{1}, y_{2} v_{2}, y_{3} v_{3}\right\}$. 
- If $f\left(y_{1}\right)<f\left(y_{2}\right)$ and $f\left(y_{1}\right)<f\left(y_{3}\right)$, then $E\left(T_{1} \cap H_{i}^{+}\right)=\left\{x_{1} v_{1}, x_{2} v_{2}, v_{2} v_{3}\right\}, E\left(T_{2} \cap H_{i}^{+}\right)=\left\{x_{3} v_{3}, v_{3} v_{1}, v_{3} v_{2}\right\}, E\left(T_{3} \cap H_{i}^{+}\right)=$ $\left\{y_{1} v_{1}, v_{1} v_{2}, v_{1} v_{3}\right\}$, and $E\left(T_{4} \cap H_{i}^{+}\right)=\left\{y_{2} v_{2}, v_{2} v_{1}, y_{3} v_{3}\right\}$.

- If $f\left(y_{2}\right)<f\left(y_{1}\right)$ and $f\left(y_{2}\right)<f\left(y_{3}\right)$, then $E\left(T_{1} \cap H_{i}^{+}\right)=\left\{x_{1} v_{1}, x_{2} v_{2}, v_{1} v_{3}\right\}, E\left(T_{2} \cap H_{i}^{+}\right)=\left\{x_{3} v_{3}, v_{3} v_{1}, v_{3} v_{2}\right\}, E\left(T_{3} \cap H_{i}^{+}\right)=$ $\left\{y_{2} v_{2}, v_{2} v_{1}, v_{2} v_{3}\right\}$, and $\left.E\left(T_{4}\right) \cap H_{i}^{+}\right)=\left\{y_{1} v_{1}, v_{1} v_{2}, y_{3} v_{3}\right\}$.

- If $f\left(y_{3}\right)<f\left(y_{1}\right)<f\left(y_{2}\right)$, then $E\left(T_{1} \cap H_{i}^{+}\right)=\left\{x_{1} v_{1}, x_{2} v_{2}, v_{1} v_{3}\right\}, E\left(T_{2} \cap H_{i}^{+}\right)=\left\{x_{3} v_{3}, v_{3} v_{1}, v_{3} v_{2}\right\}, E\left(T_{3} \cap H_{i}^{+}\right)=$ $\left\{y_{1} v_{1}, v_{1} v_{2}, y_{3} v_{3}\right\}$, and $E\left(T_{4} \cap H_{i}^{+}\right)=\left\{y_{2} v_{2}, v_{2} v_{1}, v_{2} v_{3}\right\}$.

- If $f\left(y_{3}\right)<f\left(y_{2}\right)<f\left(y_{1}\right)$, then $E\left(T_{1} \cap H_{i}^{+}\right)=\left\{x_{1} v_{1}, x_{2} v_{2}, v_{2} v_{3}\right\}, E\left(T_{2} \cap H_{i}^{+}\right)=\left\{x_{3} v_{3}, v_{3} v_{1}, v_{3} v_{2}\right\}, E\left(T_{3} \cap H_{i}^{+}\right)=$ $\left\{y_{2} v_{2}, v_{2} v_{1}, y_{3} v_{3}\right\}$, and $E\left(T_{4} \cap H_{i}^{+}\right)=\left\{y_{1} v_{1}, v_{1} v_{2}, v_{1} v_{3}\right\}$.

We can now show that $T_{1}, T_{2}, T_{3}$ and $T_{4}$ are spanning trees of $G$.

(6.8) Lemma. For every $i=1, \ldots, t, T_{1} \cap G_{i}$ and $T_{2} \cap G_{i}$ are spanning trees of $G_{i}$.

Proof. Note that every $v \in V(G)-\{r\}$ is an internal vertex of some chain $H_{i}$ in $\mathcal{C}$ The result follows by induction on $i$, with the help of (1) of Lemma (6.2), (1) and (2) of Lemma (6.3), (1) and (2) of Lemma (6.4), (1) and (2) of Lemma (6.5), (1) and (2) of Lemma (6.6), and Lemma (6.7).

(6.9) Lemma. For every $i=t, \ldots, 1, T_{3} \cap \bar{G}_{i}$ and $T_{4} \cap \bar{G}_{i}$ are spanning trees of $\bar{G}_{i}$.

Proof. The result follows by induction on $t-i$ with the help of (2) of Lemma (6.2), (3) and (4) of Lemma (6.3), (3) and (4) of Lemma (6.4), (3) and (4) of Lemma (6.5), (3) and (4) of Lemma (6.6), and Lemma (6.7).

Lemmas (6.8) and (6.9) imply the following.

(6.10) Corollary. $T_{1}, T_{2}, T_{3}, T_{4}$ are spanning trees of $G$.

Now we proceed to show that $T_{1}, T_{2}, T_{3}, T_{4}$ are independent spanning trees of $G$ rooted at $r$. The proof consists of several lemmas.

(6.11) Lemma. For any $1 \leq i \leq t$ and for any $v \in I\left(H_{i}\right)-\{r\}$, there exist vertices $z_{1}, z_{2}, z_{3}, z_{4}$ such that

(1) $z_{1}, z_{2} \in V\left(G_{i-1}\right)$, and, either $z_{1}=z_{2}=r$, or $g\left(z_{1}\right)<g\left(z_{2}\right)$ (and $g\left(z_{1}\right)<g(v)<g\left(z_{2}\right)$ if $v \in D)$,

(2) $z_{3}, z_{4} \in V\left(\bar{G}_{i}\right)$, and, either $z_{3}=z_{4}=r$, or $f\left(z_{3}\right)<f\left(z_{4}\right)$ (and $f\left(z_{3}\right)<f(v)<f\left(z_{4}\right)$ if $\left.v \in D^{\prime}\right)$, and

(3) $T_{i}\left[z_{i}, v\right], i=1,2,3,4$, are internally disjoint paths in $G$, and $V\left(T_{i}\left[z_{i}, v\right]\right)-z_{i} \subseteq I\left(H_{i}\right)$.

Proof. Let $1 \leq i \leq t$ and $v \in I\left(H_{i}\right)-\{r\}$. We consider the four cases of Algorithm Trees.

Case 1: $H_{i}$ is an elementary $G_{i-1}$-chain in $G$.

In this case, $2 \leq i \leq t-1$. Let $H_{i}:=v_{0} B_{1} v_{1} B_{2} v_{2}$, with $g\left(v_{0}\right)<g\left(v_{2}\right)$. This is the same as in Case 1 of Algorithm Trees. Then $v_{1}, v_{2} \in V\left(G_{i-1}\right), v=v_{1}$, and by Case 1 of Algorithm Numbering $g$, we have $g\left(v_{0}\right)<g\left(v_{1}\right)<g\left(v_{2}\right)$. By Lemma (6.2), there exist $v_{0}^{\prime}, v_{2}^{\prime} \in V\left(\bar{G}_{i}\right)$ with $f\left(v_{0}^{\prime}\right)<f\left(v_{2}^{\prime}\right)$ such that $v_{0} v \in E\left(T_{1}\right), v_{2} v \in E\left(T_{2}\right), v_{0}^{\prime} v \in E\left(T_{3}\right)$ and $v_{2}^{\prime} v \in E\left(T_{4}\right)$. By Case 1 
of Algorithm Numbering $f, f\left(v_{0}^{\prime}\right)<f\left(v_{1}\right)<f\left(v_{2}^{\prime}\right)$. Thus, the result follows by taking $z_{1}:=v_{0}$, $z_{2}:=v_{2}, z_{3}:=v_{0}^{\prime}$, and $z_{4}:=v_{2}^{\prime}$.

Case 2: $i=1$ or $H_{i}$ is an up $G_{i-1}$-chain in $G$, but not an elementary $G_{i-1}$-chain.

Let $H_{i}:=v_{0} B_{1} v_{1} \ldots v_{k-1} B_{k} v_{k}$, with $v_{0}=v_{k}=r$ when $i=1$, and $g\left(v_{0}\right)<g\left(v_{k}\right)$ when $i \neq 1$. For each 2-connected $B_{j}$, let $u_{j}, w_{j}$ denote the terminals of $B_{j}^{+}$other than $v_{j-1}, v_{j}$, with $f\left(u_{j}\right)<f\left(w_{j}\right)$, and let $T_{v_{j-1}}^{j}, T_{v_{j}}^{j}, T_{u_{j}}^{j}, T_{w_{j}}^{j}$ denote the trees rooted respectively at $v_{j-1}, v_{j}, u_{j}, w_{j}$ in the independent spanning $\left\{v_{j-1}, v_{j}, u_{j}, w_{j}\right\}$-system of $B_{j}^{+}$computed in (4.5). This is the same as in Case 2 of Algorithm Trees,

Let $j \in\{1, \ldots, k-1\}$. If $i=1$, then by (1) and (2) of Lemma (6.3), $T_{1}\left[r, v_{j}\right] \subseteq \bigcup_{l=1}^{j} B_{l}$ and $T_{2}\left[r, v_{j}\right] \subseteq \bigcup_{l=j+1}^{k} B_{l}$. If $i \neq 1$, then $v_{j}$ is a cut vertex of $H_{i}$, and hence, by (1) and (2) of Lemma (6.4), $T_{1}\left[v_{0}, v_{j}\right] \subseteq \bigcup_{l=1}^{j} B_{l}$ and $T_{2}\left[v_{k}, v_{j}\right] \subseteq \bigcup_{l=j+1}^{k} B_{l}$.

First, let us consider the case when $v \neq v_{j}$ for $j=1, \ldots, k-1$. Thus, there exists some $j$, $1 \leq j \leq k$, such that $B_{j}$ is 2-connected and $v \in V\left(B_{j}\right)-\left\{v_{j-1}, v_{j}\right\}$. By Case 2 of Algorithm Numbering $g$, we know that $g\left(v_{0}\right) \leq g\left(v_{j-1}\right)<g\left(v_{j}\right) \leq g\left(v_{k}\right)$, and if $v \in D$ then $g\left(v_{0}\right) \leq$ $g\left(v_{j-1}\right)<g(v)<g\left(v_{j}\right) \leq g\left(v_{k}\right)$. Furthermore, $T_{v_{j-1}}^{j}\left[v_{j-1}, v\right], T_{v_{j}}^{j}\left[v_{j}, v\right], T_{u_{j}}^{j}\left[u_{j}, v\right], T_{w_{j}}^{j}\left[w_{j}, v\right]$ are internally disjoint, because $\left\{T_{v_{j-1}}^{j}, T_{v_{j}}^{j}, T_{u_{j}}^{j}, T_{w_{j}}^{j}\right\}$ is an independent spanning $\left\{v_{j-1}, v_{j}, u_{j}, w_{j}\right\}-$ system of $B_{j}^{+}$. By the construction in Case 2 of Algorithm Trees, $T_{1}\left[v_{j-1}, v\right]=T_{v_{j-1}}^{j}\left[v_{j-1}, v\right]$, $T_{2}\left[v_{j}, v\right]=T_{v_{j}}^{j}\left[v_{j}, v\right], T_{3}\left[u_{j}, v\right]=T_{u_{j}}^{j}\left[u_{j}, v\right]$, and $T_{4}\left[w_{j}, v\right]=T_{w_{j}}^{j}\left[w_{j}, v\right]$. By Case 3 of Algorithm Numbering $f$, if $v \in D^{\prime}$ then $f\left(u_{j}\right)<f(v)<f\left(w_{j}\right)$. Moreover, $T_{1}\left[v_{0}, v_{j-1}\right] \subseteq \bigcup_{l=1}^{j-1} B_{l}$ and $T_{2}\left[v_{k}, v_{j}\right] \subseteq \bigcup_{l=j+1}^{k} B_{l}$. Let $z_{1}:=v_{0}, z_{2}:=v_{k}, z_{3}:=u_{j}$ and $z_{4}:=w_{j}$. Clearly, (1)-(3) hold.

So assume that $v=v_{j}$ for some $j, 1 \leq j \leq k-1$. Let $z_{1}:=v_{0}$ and $z_{2}:=v_{k}$. Then by Case 2 of Algorithm Numbering $g, g\left(z_{1}\right)<g(v)<g\left(z_{2}\right)$. We will define $z_{3}$ and $z_{4}$ and prove that (1)-(3) hold. We do this by analyzing how Algorithm Trees chooses the neighbors $p_{3}, p_{4}$ of $v_{j}$ in the trees $T_{3}, T_{4}$, respectively.

Subcase 2.1: $\quad B_{j}$ and $B_{j+1}$ are trivial (Subcase 2.1 in Algorithm Trees).

Then Algorithm Trees chooses neighbors $p_{3}, p_{4}$ of $v_{j}$ in $V\left(\bar{G}_{i}\right)$ with $f\left(p_{3}\right)$ minimum (and hence $\left.f\left(p_{3}\right)<f\left(p_{4}\right)\right)$. If $v_{j} \in D^{\prime}$ then by Case 3 of Algorithm Numbering $f$, we have $f\left(p_{3}\right)<f\left(v_{j}\right)<$ $f\left(p_{4}\right)$. Let $z_{3}:=p_{3}$ and $z_{4}:=p_{4}$. Clearly, (1)-(3) hold.

Subcase 2.2: $\quad B_{j}$ is 2-connected and $B_{j+1}$ is trivial (Subcase 2.2 in Algorithm Trees).

- If $v_{j}$ has no neighbor in $V\left(\bar{G}_{i}\right)$, then Algorithm Trees chooses three neighbors $p_{1}, p_{3}, p_{4}$ of $v_{j}$ in $B_{j}$ such that $T_{v_{j-1}}^{j}\left[v_{j-1}, p_{1}\right], T_{u_{j}}^{j}\left[u_{j}, p_{3}\right]$ and $T_{w_{j}}^{j}\left[w_{j}, p_{4}\right]$ are disjoint. By construction, $T_{1}\left[v_{j-1}, v_{j}\right]=T_{v_{j-1}}^{j}\left[v_{j-1}, p_{1}\right]+\left\{v_{j}, v_{j} p_{1}\right\}, T_{3}\left[u_{j}, v_{j}\right]=T_{u_{j}}^{j}\left[u_{j}, p_{3}\right]+\left\{v_{j}, v_{j} p_{3}\right\}$ and $T_{4}\left[w_{j}, v_{j}\right]=T_{w_{j}}^{j}\left[w_{j}, p_{4}\right]+\left\{v_{j}, v_{j} p_{4}\right\}$. Moreover, $T_{1}\left[v_{0}, v_{j-1}\right] \subseteq \bigcup_{l=1}^{j-1} B_{l}$ and $T_{2}\left[v_{k}, v_{j}\right] \subseteq$ $\bigcup_{l=j+1}^{k} B_{l}$. Therefore, $T_{1}\left[v_{0}, v_{j}\right], T_{2}\left[v_{k}, v_{j}\right], T_{3}\left[u_{j}, v_{j}\right]$ and $T_{4}\left[w_{j}, v_{j}\right]$ are internally disjoint. If $v_{j} \in D^{\prime}$ then by Case of Algorithm Numbering $f$, we have $j=k-1$ and $f\left(u_{j}\right)<f(v)<f\left(w_{j}\right)$. Let $z_{3}:=u_{j}$ and $z_{4}:=w_{j}$. Clearly, (1)-(3) hold.

- If $v_{j}$ has a neighbor in $V\left(\bar{G}_{i}\right)$, then Algorithm Trees chooses a vertex $x \in N_{G}\left(v_{j}\right) \cap V\left(\bar{G}_{i}\right)$ with $f(x)$ minimum.

- If $f(x)>f\left(u_{j}\right)$, then the algorithm chooses neighbors $p_{1}, p_{3}$ of $v_{j}$ in $B_{j}$ such that $T_{v_{j-1}}^{j}\left[v_{j-1}, p_{1}\right]$ and $T_{u_{j}}^{j}\left[u_{j}, p_{3}\right]$ are disjoint. By construction, $T_{1}\left[v_{j-1}, v_{j}\right]=$ $T_{v_{j-1}}^{j}\left[v_{j-1}, p_{1}\right]+\left\{v_{j}, v_{j} p_{1}\right\}, T_{3}\left[u_{j}, v_{j}\right]=T_{u_{j}}^{j}\left[u_{j}, p_{3}\right]+\left\{v_{j}, v_{j} p_{3}\right\}$, and $T_{4}\left[x, v_{j}\right]$ is induced by the edge $x v_{j}$. Moreover, $T_{1}\left[v_{0}, v_{j-1}\right] \subseteq \bigcup_{l=1}^{j-1} B_{l}$, and $T_{2}\left[v_{k}, v_{j}\right] \subseteq \bigcup_{l=j+1}^{k} B_{l}$. Therefore, $T_{1}\left[v_{0}, v_{j}\right], T_{2}\left[v_{k}, v_{j}\right], T_{3}\left[u_{j}, v_{j}\right]$ and $T_{4}\left[x, v_{j}\right]$ are internally disjoint. If $v_{j} \in D^{\prime}$ then by Case of Algorithm Numbering $f$, we have $f\left(u_{j}\right)<f(v)<f(x)$. Let $z_{3}:=u_{j}$ and $z_{4}:=x$. Clearly, (1)-(3) hold. 
- If $f(x) \leq f\left(u_{j}\right)$, then Algorithm Trees chooses neighbors $p_{1}, p_{4}$ of $v_{j}$ in $B_{j}$ such that $T_{v_{j-1}}^{j}\left[v_{j-1}, p_{1}\right]$ and $T_{w_{j}}^{j}\left[w_{j}, p_{4}\right]$ are disjoint. By construction, $T_{1}\left[v_{j-1}, v_{j}\right]=$ $T_{v_{j-1}}^{j}\left[v_{j-1}, p_{1}\right]+\left\{v_{j}, v_{j} p_{1}\right\}, T_{4}\left[w_{j}, v_{j}\right]=T_{w_{j}}^{j}\left[w_{j}, p_{4}\right]+\left\{v_{j}, v_{j} p_{4}\right\}$, and $T_{3}\left[x, v_{j}\right]$ is induced by the edge $x v_{j}$. Moreover, $T_{1}\left[v_{0}, v_{j-1}\right] \subseteq \bigcup_{l=1}^{j-1} B_{l}$, and $T_{2}\left[v_{k}, v_{j}\right] \subseteq \bigcup_{l=j+1}^{k} B_{l}$. Therefore, $T_{1}\left[v_{0}, v_{j}\right], T_{2}\left[v_{k}, v_{j}\right], T_{3}\left[u_{j}, v_{j}\right]$ and $T_{4}\left[x, v_{j}\right]$ are internally disjoint. If $v_{j} \in D^{\prime}$ then by Case of Algorithm Numbering $f$, we have $f(x)<f(v)<f\left(w_{j}\right)$. Let $z_{3}:=x$ and $z_{4}:=w_{j}$. Clearly, (1)-(3) hold.

Subcase 2.3: $\quad B_{j}$ is trivial and $B_{j+1}$ is 2-connected (Subcase 2.3 in Algorithm Trees).

In this case, if $v_{j} \in D^{\prime}$ then $j=1$ by Case 3 of Algorithm Numbering $f$. The arguments for the proof are similar to Subcase 2.2 and we only indicate the choice of $z_{3}$ and $z_{4}$. In each case below, one can show that (1)-(3) hold for the corresponding choice of $z_{3}, z_{4}$.

- If $v_{j}$ has no neighbor in $V\left(\bar{G}_{i}\right)$, then let $z_{3}:=u_{j+1}$ and $z_{4}:=w_{j+1}$.

- If $v_{j}$ has a neighbor in $V\left(\bar{G}_{i}\right)$, then Algorithm Trees chooses a vertex $x \in N_{G}\left(v_{j}\right) \cap V\left(\bar{G}_{i}\right)$ with $f(x)$ minimum.

- If $f(x)>f\left(u_{j+1}\right)$, then let $z_{3}:=u_{j+1}$ and $z_{4}:=x$.

- If $f(x) \leq f\left(u_{j+1}\right)$, then let $z_{3}:=x$ and let $z_{4}:=w_{j+1}$.

Subcase 2.4: $\quad$ Both $B_{j}$ and $B_{j+1}$ are 2-connected (Subcase 2.4 in Algorithm Trees).

Since $G$ is 4 -connected and both $\left(B_{j}^{+}, v_{j-1}, u_{j}, v_{j}, w_{j}\right)$ and $\left(B_{j+1}^{+}, v_{j}, u_{j+1}, v_{j+1}, w_{j+1}\right)$ are planar, $v_{j} \notin N_{B_{j}}\left(v_{j-1}\right) \cup N_{B_{j+1}}\left(v_{j+1}\right)$. Hence $v_{j} \notin D^{\prime}$ by Case 3 of Algorithm Numbering $f$. Note that $f\left(u_{j}\right)<f\left(w_{j+1}\right)$ or $f\left(u_{j+1}\right)<f\left(w_{j}\right)$.

- If $f\left(u_{j}\right)<f\left(w_{j+1}\right)$, then Algorithm Trees chooses neighbors $p_{1}, p_{3}$ of $v_{j}$ in $B_{j}$ such that $T_{v_{j-1}}^{j}\left[v_{j-1}, p_{1}\right], T_{u_{j}}^{j}\left[u_{j}, p_{3}\right]$ are disjoint, and neighbors $p_{2}, p_{4}$ of $v_{j}$ in $B_{j+1}$ such that $T_{v_{j+1}}^{j}\left[v_{j+1}, p_{2}\right], T_{w_{j+1}}^{j}\left[w_{j+1}, p_{4}\right]$ are disjoint. By construction, $T_{1}\left[v_{j-1}, v_{j}\right]=T_{v_{j-1}}^{j}\left[v_{j-1}, p_{1}\right]+$ $\left\{v_{j}, v_{j} p_{1}\right\}, T_{3}\left[u_{j}, v_{j}\right]=T_{u_{j}}^{j}\left[u_{j}, p_{3}\right]+\left\{v_{j}, v_{j} p_{3}\right\}, T_{2}\left[v_{j+1}, v_{j}\right]=T_{v_{j+1}}^{j+1}\left[v_{j+1}, p_{2}\right]+\left\{v_{j}, v_{j} p_{2}\right\}$ and $T_{4}\left[w_{j+1}, v_{j}\right]=T_{w_{j+1}}^{j+1}\left[w_{j+1}, p_{4}\right]+\left\{v_{j}, v_{j} p_{4}\right\}$. Moreover, $T_{1}\left[v_{0}, v_{j-1}\right] \subseteq \bigcup_{l=1}^{j-1} B_{l}$, and $T_{2}\left[v_{k}, v_{j+1}\right] \subseteq \bigcup_{l=j+2}^{k} B_{l}$. Thus, $T_{1}\left[v_{0}, v_{j}\right], T_{2}\left[v_{k}, v_{j}\right], T_{3}\left[u_{j}, v_{j}\right]$ and $T_{4}\left[w_{j+1}, v_{j}\right]$ are internally disjoint. Let $z_{3}:=u_{j}$ and $z_{4}:=w_{j+1}$. Clearly (1)-(3) hold.

- If $f\left(u_{j}\right) \geq f\left(w_{j+1}\right.$, then $f\left(u_{j+1}\right)<f\left(w_{j}\right)$, and Algorithm Trees chooses neighbors $p_{1}, p_{4}$ of $v_{j}$ in $B_{j}$ such that $T_{v_{j-1}}^{j}\left[v_{j-1}, p_{1}\right], T_{w_{j}}^{j}\left[w_{j}, p_{4}\right]$ are disjoint, and neighbors $p_{2}, p_{3}$ of $v_{j}$ in $B_{j+1}$ such that $T_{v_{j+1}}^{j+1}\left[v_{j+1}, p_{2}\right], T_{u_{j+1}}^{j+1}\left[u_{j+1}, p_{3}\right]$ are disjoint. Let $z_{3}:=u_{j+1}$ and $z_{4}:=w_{j}$. One can show as in the above paragraph that $T_{1}\left[v_{0}, v_{j}\right], T_{2}\left[v_{k}, v_{j}\right], T_{3}\left[u_{j+1}, v_{j}\right]$ and $T_{4}\left[w_{j}, v_{j}\right]$ are internally disjoint and (1)-(3) hold.

Case 3: $i=t$ or $H_{i}$ is a down $G_{i-1}$-chain in $G$, but not an elementary $G_{i-1}$-chain.

Let $H_{i}:=v_{0} B_{1} v_{1} \ldots v_{k-1} B_{k} v_{k}$, with $v_{0}=v_{k}=r$ when $i=t$, and $f\left(v_{0}\right)<f\left(v_{k}\right)$ when $i \neq t$. For each 2-connected $B_{j}$ let $u_{j}, w_{j}$ denote the terminals of $B_{j}^{+}$other than $v_{j-1}, v_{j}$, with $g\left(u_{j}\right)<g\left(w_{j}\right)$, and let $T_{v_{j-1}}^{j}, T_{v_{j}}^{j}, T_{u_{j}}^{j}, T_{w_{j}}^{j}$ denote the trees rooted respectively at $v_{j-1}, v_{j}, u_{j}, w_{j}$ in the independent spanning $\left\{v_{j-1}, v_{j}, u_{j}, w_{j}\right\}$-system of $B_{j}^{+}$computed in (4.5). This is the same as in Case 3 of Algorithm Trees.

Let $j \in\{1, \ldots, k-1\}$. If $i=t$, then by (3) and (4) of Lemma (6.5), $T_{3}\left[v_{0}, v_{j}\right] \subseteq \bigcup_{l=1}^{j} B_{l}$ and $T_{4}\left[v_{k}, v_{j}\right] \subseteq \bigcup_{l=j+1}^{k} B_{l}$. If $i \neq t$ then $v_{j}$ is a cut vertex of $H_{i}$, and hence, by (3) and (4) of Lemma (6.6), $T_{3}\left[v_{0}, v_{j}\right] \subseteq \bigcup_{l=1}^{j} B_{l}$ and $T_{4}\left[v_{k}, v_{j}\right] \subseteq \bigcup_{l=j+1}^{k} B_{l}$.

First, let us consider the case when $v \neq v_{j}$ for $j=1, \ldots, k-1$. Thus, there exists some $j$, $1 \leq j \leq k$, such that $B_{j}$ is 2-connected and $v \in V\left(B_{j}\right)-\left\{v_{j-1}, v_{j}\right\}$. By Case 2 of Algorithm Numbering $f$, we know that $f\left(v_{0}\right) \leq f\left(v_{j-1}\right)<f\left(v_{j}\right) \leq f\left(v_{k}\right)$, and if $v_{j} \in D^{\prime}$ then $f\left(v_{0}\right) \leq f\left(v_{j-1}\right)<$ 
$f(v)<f\left(v_{j}\right) \leq f\left(v_{k}\right)$. Furthermore, $T_{v_{j-1}}^{j}\left[v_{j-1}, v\right], T_{v_{j}}^{j}\left[v_{j}, v\right], T_{u_{j}}^{j}\left[u_{j}, v\right], T_{w_{j}}^{j}\left[w_{j}, v\right]$, are internally disjoint because $\left\{T_{v_{j-1}}^{j}, T_{v_{j}}^{j}, T_{u_{j}}^{j}, T_{w_{j}}^{j}\right\}$ is an independent spanning $\left\{v_{j-1}, v_{j}, u_{j}, w_{j}\right\}$-system of $B_{j}^{+}$. By the construction in Case 3 of Algorithm Trees, $T_{1}\left[u_{j}, v\right]=T_{u_{j}}^{j}\left[u_{j}, v\right], T_{2}\left[w_{j}, v\right]=T_{w_{j}}^{j}\left[w_{j}, v\right]$, $T_{3}\left[v_{j-1}, v\right]=T_{v_{j-1}}^{j}\left[v_{j-1}, v\right]$ and $T_{4}\left[v_{j}, v\right]=T_{v_{j}}^{j}\left[v_{j}, v\right]$. Moreover, $T_{3}\left[v_{0}, v_{j-1}\right] \subseteq \bigcup_{l=1}^{j-1} B_{l}$ and $T_{4}\left[v_{k}, v_{j}\right] \subseteq \bigcup_{l=j+1}^{k} B_{l}$. Let $z_{1}:=u_{j}, z_{2}:=w_{j}, z_{3}:=v_{0}$ and $z_{4}:=v_{k}$. Clearly (1)-(3) hold.

So assume that $v=v_{j}$ for some $j, 1 \leq j \leq k-1$. Let $z_{3}:=v_{0}$ and $z_{4}:=v_{k}$. By Case 2 of Algorithm Numbering $f$, we have $f\left(z_{2}\right)<f(v)<f\left(z_{4}\right)$. We will define $z_{1}$ and $z_{2}$ and prove that (1)-(3) hold. We do this by analyzing how Algorithm Trees chooses the neighbors $p_{1}, p_{2}$ of $v_{j}$ in the trees $T_{1}, T_{2}$, respectively.

Subcase 3.1: $B_{j}$ and $B_{j+1}$ are trivial (Subcase 3.1 in Algorithm Trees).

Then Algorithm Trees chooses neighbors $p_{1}, p_{2}$ of $v_{j}$ in $V\left(G_{i-1}\right)$ with $g\left(p_{1}\right)$ minimum (and so $\left.g\left(p_{1}\right)<g\left(p_{2}\right)\right)$. By Subcase 3.3 of Algorithm Numbering $g$, we have $g\left(p_{1}\right)<g(v)<g\left(p_{2}\right)$. Let $z_{1}:=p_{1}$ and $z_{2}:=p_{2}$. Clearly, (1)-(3) hold.

Subcase 3.2: $\quad B_{j}$ is 2-connected and $B_{j+1}$ is trivial (Subcase 3.2 in Algorithm Trees).

- If $v_{j}$ has no neighbor in $V\left(G_{i-1}\right)$, then Algorithm Trees chooses three neighbors $p_{1}, p_{2}, p_{3}$ of $v_{j}$ in $B_{j}$ such that $T_{u_{j}}^{j}\left[u_{j}, p_{1}\right], T_{w_{j}}^{j}\left[w_{j}, p_{2}\right]$ and $T_{v_{j-1}}^{j}\left[v_{j-1}, p_{3}\right]$ are disjoint. By construction, $T_{1}\left[u_{j}, v_{j}\right]=T_{u_{j}}^{j}\left[u_{j}, p_{1}\right]+\left\{v_{j}, v_{j} p_{1}\right\}, T_{2}\left[w_{j}, v_{j}\right]=T_{w_{j}}^{j}\left[w_{j}, p_{2}\right]+\left\{v_{j}, v_{j} p_{2}\right\}$ and $T_{3}\left[v_{j-1}, v_{j}\right]=$ $T_{v_{j-1}}^{j}\left[v_{j-1}, p_{3}\right]+\left\{v_{j}, v_{j} p_{3}\right\}$. Moreover, $T_{3}\left[v_{0}, v_{j-1}\right] \subseteq \bigcup_{l=1}^{j-1} B_{l}$ and $T_{4}\left[v_{k}, v_{j}\right] \subseteq \bigcup_{l=j+1}^{k} B_{l}$. Therefore, $T_{1}\left[u_{j}, v_{j}\right], T_{2}\left[w_{j}, v_{j}\right], T_{3}\left[v_{0}, v_{j}\right]$ and $T_{4}\left[v_{k}, v_{j}\right]$ are internally disjoint. In this case, if $v_{j} \in D$ then by Case 3 of Algorithm Numbering $g$, we have $j=k-1$ and $g\left(u_{j}\right)<g(v)<$ $g\left(w_{j}\right)$. Let $z_{1}:=u_{j}$ and $z_{2}:=w_{j}$. Clearly, (1)-(3) hold.

- If $v_{j}$ has a neighbor in $V\left(G_{i-1}\right)$, then Algorithm Trees chooses a vertex $x \in N_{G}\left(v_{j}\right) \cap$ $V\left(G_{i-1}\right)$ with $g(x)$ minimum.

- If $g(x)>g\left(u_{j}\right)$, then the algorithm chooses neighbors $p_{1}, p_{3}$ of $v_{j}$ in $B_{j}$ such that $T_{u_{j}}^{j}\left[u_{j}, p_{1}\right]$ and $T_{v_{j-1}}^{j}\left[v_{j-1}, p_{3}\right]$ are disjoint. By construction, $T_{1}\left[u_{j}, v_{j}\right]=T_{u_{j}}^{j}\left[u_{j}, p_{1}\right]+$ $\left\{v_{j}, v_{j} p_{1}\right\}, T_{3}\left[v_{j-1}, v_{j}\right]=T_{v_{j-1}}^{j}\left[v_{j-1}, p_{3}\right]+\left\{v_{j}, v_{j} p_{3}\right\}$, and $T_{2}\left[x, v_{j}\right]$ is induced by the edge $x v_{j}$. Moreover, $T_{3}\left[v_{0}, v_{j-1}\right] \subseteq \bigcup_{l=1}^{j-1} B_{l}$ and $T_{4}\left[v_{k}, v_{j}\right] \subseteq \bigcup_{l=j+1}^{k} B_{l}$. Therefore, $T_{1}\left[u_{j}, v_{j}\right], T_{2}\left[x, v_{j}\right], T_{3}\left[v_{0}, v_{j}\right]$ and $T_{4}\left[v_{k}, v_{j}\right]$ are internally disjoint. If $v_{j} \in D$ then by Case 3 of Algorithm Numbering $g$, we have $j=k-1$ and $g\left(u_{j}\right)<g(v)<g(x)$. Let $z_{1}:=u_{j}$ and $z_{4}:=x$. Clearly, (1)-(3) hold.

- If $g(x) \leq g\left(u_{j}\right)$, then Algorithm Trees chooses neighbors $p_{2}, p_{3}$ of $v_{j}$ in $B_{j}$ such that $T_{w_{j}}^{j}\left[w_{j}, p_{2}\right]$ and $T_{v_{j-1}}^{j}\left[v_{j-1}, p_{3}\right]$ are disjoint. By construction, $T_{2}\left[w_{j}, v_{j}\right]=T_{w_{j}}^{j}\left[w_{j}, p_{2}\right]+$ $\left\{v_{j}, v_{j} p_{2}\right\}, T_{3}\left[v_{j-1}, v_{j}\right]=T_{v_{j-1}}^{j}\left[v_{j-1}, p_{3}\right]+\left\{v_{j}, v_{j} p_{3}\right\}$, and $T_{1}\left[x, v_{j}\right]$ is induced by the edge $x v_{j}$. Moreover, $T_{3}\left[v_{0}, v_{j-1}\right] \subseteq \bigcup_{l=1}^{j-1} B_{l}$ and $T_{4}\left[v_{k}, v_{j}\right] \subseteq \bigcup_{l=j+1}^{k} B_{l}$. Therefore, $T_{1}\left[x, v_{j}\right], T_{2}\left[w_{j}, v_{j}\right], T_{3}\left[v_{0}, v_{j}\right]$ and $T_{4}\left[v_{k}, v_{j}\right]$ are internally disjoint. If $v_{j} \in D$ then by Case 3 of Algorithm Numbering $g$, we have $j=k-1$ and $g(x)<g(v)<g\left(w_{j}\right)$. Let $z_{1}:=x$ and $z_{2}:=w_{j}$. Clearly, (1)-(3) hold.

Subcase 3.3: $\quad B_{j}$ is trivial and $B_{j+1}$ is 2-connected (Subcase 3.3 in Algorithm Trees).

In this case, if $v_{j} \in D$ then $j=1$ by Case 3 of Algorithm Numbering $g$. The arguments for this case are similar to Subcase 3.2 and we only indicate the choice of $z_{1}$ and $z_{2}$. In each case below, one can show that (1)-(3) hold for the corresponding choice of $z_{1}, z_{2}$.

- If $v_{j}$ has no neighbor in $V\left(G_{i-1}\right)$, then let $z_{1}:=u_{j+1}$ and $z_{2}:=w_{j+1}$.

- If $v_{j}$ has a neighbor in $V\left(G_{i-1}\right)$, then Algorithm Trees chooses a vertex $x \in N_{G}\left(v_{j}\right) \cap$ $V\left(G_{i-1}\right)$ with $g(x)$ minimum.

- If $g(x)>g\left(u_{j+1}\right)$, then let $z_{1}:=u_{j+1}$ and $z_{2}:=x$. 
- If $g(x) \leq g\left(u_{j+1}\right)$, then let $z_{1}:=x$ and $z_{2}:=w_{j+1}$.

Subcase 3.4: $\quad B_{j}$ and $B_{j+1}$ are 2-connected (Subcase 3.4 in Algorithm Trees).

Since $G$ is 4 -connected and both $\left(B_{j}^{+}, v_{j-1}, u_{j}, v_{j}, w_{j}\right)$ and $\left(B_{j+1}^{+}, v_{j}, u_{j+1}, v_{j+1}, w_{j+1}\right)$ are planar, $v_{j} \notin N_{B_{j}}\left(v_{j-1}\right) \cup N_{B_{j+1}}\left(v_{j+1}\right)$. So by Case 3 of Algorithm Numbering $g, v_{j} \notin D$. Note that $g\left(u_{j}\right)<g\left(w_{j+1}\right)$ or $g\left(u_{j+1}\right)<g\left(w_{j}\right)$.

- If $g\left(u_{j}\right)<g\left(w_{j+1}\right)$, then Algorithm Trees chooses neighbors $p_{1}, p_{3}$ of $v_{j}$ in $B_{j}$ such that $T_{u_{j}}^{j}\left[u_{j}, p_{1}\right]$ and $T_{v_{j-1}}^{j}\left[v_{j-1}, p_{3}\right]$ are disjoint, and neighbors $p_{2}, p_{4}$ of $v_{j}$ in $B_{j+1}$ such that $T_{w_{j+1}}^{j+1}\left[w_{j+1}, p_{2}\right]$ and $T_{v_{j+1}}^{j+1}\left[v_{j+1}, p_{4}\right]$ are disjoint. By construction, $T_{1}\left[u_{j}, v_{j}\right]=T_{u_{j}}^{j}\left[u_{j}, p_{1}\right]+$ $\left\{v_{j}, v_{j} p_{1}\right\}, T_{3}\left[v_{j-1}, v_{j}\right]=T_{v_{j-1}}^{j}\left[v_{j-1}, p_{3}\right]+\left\{v_{j}, v_{j} p_{3}\right\}, T_{2}\left[w_{j+1}, v_{j}\right]=T_{w_{j+1}}^{j+1}\left[w_{j+1}, p_{2}\right]+$ $\left\{v_{j}, v_{j} p_{2}\right\}$, and $T_{4}\left[v_{j+1}, v_{j}\right]=T_{v_{j+1}}^{j+1}\left[v_{j+1}, p_{4}\right]+\left\{v_{j}, v_{j} p_{4}\right\}$. Moreover, $T_{3}\left[v_{0}, v_{j-1}\right] \subseteq \bigcup_{l=1}^{j-1} B_{l}$ and $T_{4}\left[v_{k}, v_{j+1}\right] \subseteq \bigcup_{l=j+2}^{k} B_{l}$. Thus, $T_{1}\left[u_{j}, v_{j}\right], T_{2}\left[w_{j+1}, v_{j}\right], T_{3}\left[v_{0}, v_{j}\right]$ and $T_{4}\left[v_{k}, v_{j}\right]$ are internally disjoint. Let $z_{1}:=u_{j}$ and $z_{2}:=w_{j+1}$. Clearly (1)-(3) hold.

- If $g\left(u_{j}\right) \geq g\left(w_{j+1}\right)$, then $g\left(u_{j+1}\right)<g\left(w_{j}\right)$, and Algorithm Trees chooses neighbors $p_{2}, p_{3}$ of $v_{j}$ in $B_{j}$ such that $T_{w_{j}}^{j}\left[w_{j}, p_{2}\right]$ and $T_{v_{j-1}}^{j}\left[v_{j-1}, p_{3}\right]$ are disjoint, and neighbors $p_{1}, p_{4}$ of $v_{j}$ in $B_{j+1}$ such that $T_{u_{j+1}}^{j+1}\left[u_{j+1}, p_{1}\right]$ and $T_{v_{j+1}}^{j+1}\left[v_{j+1}, p_{4}\right]$ are disjoint. Let $z_{1}:=u_{j+1}$ and $z_{2}:=w_{j}$. One can show as in the above paragraph that $T_{1}\left[u_{j+1}, v_{j}\right], T_{2}\left[w_{j}, v_{j}\right], T_{3}\left[v_{0}, v_{j}\right]$ and $T_{4}\left[v_{k}, v_{j}\right]$ are internally disjoint and (1)-(3) hold.

Case 4: $H_{i}$ is a triangle $G_{i-1}$-chain in $G$.

Let $I\left(H_{i}\right):=\left\{v_{1}, v_{2}, v_{3}\right\}$, let $y_{1}, y_{2}, y_{3} \in V\left(\bar{G}_{i}\right)$ such that $y_{1} v_{1}, y_{2} v_{2}, y_{3} v_{3} \in E(G)$, and let $v_{j} x_{j}$ $(j=1,2,3)$ be the legs of $H_{i}$. Assume that $v_{1}, v_{2}, v_{3}$ are labeled so that $g\left(x_{1}\right)<g\left(x_{2}\right)<g\left(x_{3}\right)$.

The proof can be done by inspecting a small number of cases (Case 4 in Algorithm Trees) and using Lemma (6.7) and Case 4 of Algorithm Numbering $g$ and Algorithm Numbering $f$. For the sake of completeness, we list for each case the choice for $z_{1}, z_{2}, z_{3}$ and $z_{4}$. The verification that they satisfy (1)-(3) is straightforward and we omit it.

- If $f\left(y_{1}\right)<f\left(y_{2}\right)$ and $f\left(y_{1}\right)<f\left(y_{3}\right)$, then let $z_{2}:=x_{3}$ and $z_{3}:=y_{1}$.

If $v=v_{1}$ then let $z_{1}:=x_{1}$ and $z_{4}:=y_{2}$.

If $v=v_{2}$ then let $z_{1}:=x_{2}$ and $z_{4}:=y_{2}$.

If $v=v_{3}$ then let $z_{1}:=x_{2}$ and $z_{4}:=y_{3}$.

- If $f\left(y_{2}\right)<f\left(y_{1}\right)$ and $f\left(y_{2}\right)<f\left(y_{3}\right)$, then let $z_{2}:=x_{3}$ and $z_{3}:=y_{2}$.

If $v=v_{1}$ then let $z_{1}:=x_{1}$ and $z_{4}:=y_{1}$.

If $v=v_{2}$ then let $z_{1}:=x_{2}$ and $z_{4}:=y_{1}$.

If $v=v_{3}$ then let $z_{1}:=x_{1}$ and $z_{4}:=y_{3}$.

- If $f\left(y_{3}\right)<f\left(y_{1}\right)<f\left(y_{2}\right)$ then let $z_{2}:=x_{3}$ and $z_{4}:=y_{2}$.

If $v=v_{1}$ then let $z_{1}:=x_{1}$ and $z_{3}:=y_{1}$.

If $v=v_{2}$ then let $z_{1}:=x_{2}$ and $z_{3}:=y_{1}$.

If $v=v_{3}$ then let $z_{1}:=x_{1}$ and $z_{3}:=y_{3}$.

- If $f\left(y_{3}\right)<f\left(y_{2}\right)<f\left(y_{1}\right)$ then let $z_{2}:=x_{3}$ and $z_{4}:=y_{1}$.

If $v=v_{1}$ then let $z_{1}:=x_{1}$ and $z_{3}:=y_{2}$.

If $v=v_{2}$ then let $z_{1}:=x_{2}$ and $z_{3}:=y_{2}$.

If $v=v_{3}$ then let $z_{1}:=x_{2}$ and $z_{3}:=y_{3}$.

This completes the proof of the lemma. 
(6.12) Lemma. Let $i \in\{1, \ldots, t-1\}$. Then for any $u, v \in D_{i}$ with $g(u)<g(v), T_{1}[r, u]$ and $T_{2}[r, v]$ are internally disjoint paths in $G_{i}$.

Proof. We will prove the lemma by induction on $i$. The basis of induction is $i=0$ with $D_{0}:=\{r\}$ and $G_{0}:=(\{r\}, \emptyset)$. So assume that $i>0$ and the lemma holds for $i-1$. We consider four cases as in Algorithm Numbering $g$.

Case 1: $H_{i}$ is an elementary $G_{i-1}$-chain in $G$.

Let $H_{i}:=v_{0} B_{1} v_{1} B_{2} v_{2}$, with $g\left(v_{0}\right)<g\left(v_{2}\right)$. By (1) of Lemma (6.2), $E\left(T_{1} \cap H_{i}\right)=\left\{v_{0} v_{1}\right\}$ and $E\left(T_{2} \cap H_{i}\right)=\left\{v_{1} v_{2}\right\}$. Recall that $D_{i}=D_{i-1} \cup\left\{v_{1}\right\}$.

If $u, v \in D_{i-1}$ and $g(u)<g(v)$, then by the induction hypothesis, $T_{1}[r, u]$ and $T_{2}[r, v]$ are internally disjoint paths in $G_{i-1}$. Thus, it suffices to prove the following: for any $u, v \in D_{i}$ with $g(u)<g(v)$ and $v_{1} \in\{u, v\}, T_{1}[r, u]$ and $T_{2}[r, v]$ are internally disjoint paths in $G_{i}$.

Assume first that $u=v_{1}$. Then $v=v_{2} \in D_{i-1}$. Since $g\left(v_{0}\right)<g\left(v_{1}\right)<g(v)$, it follows from the induction hypothesis that $T_{1}\left[r, v_{0}\right]$ and $T_{2}[r, v]$ are internally disjoint paths in $G_{i-1}$. Therefore, $T_{1}\left[r, v_{1}\right]=T_{1}\left[r, v_{0}\right]+\left\{v_{1}, v_{1} v_{0}\right\}$ and $T_{2}[r, v]$ are internally disjoint paths in $G_{i}$.

Now suppose $v=v_{1}$. Then $u \in D_{i-1}$. Since $g(u)<g\left(v_{1}\right)<g\left(v_{2}\right)$, it follows from the induction hypothesis that $T_{1}[r, u]$ and $T_{2}\left[r, v_{2}\right]$ are internally disjoint paths in $G_{i-1}$. Therefore, $T_{1}[r, u]$ and $T_{2}\left[r, v_{1}\right]=T_{2}\left[r, v_{2}\right]+\left\{v_{1}, v_{1} v_{2}\right\}$ are internally disjoint paths in $G_{i}$.

Case 2: $i=1$ or $H_{i}$ is an up $G_{i-1}$-chain in $G$, but not an elementary $G_{i-1}$-chain in $G$.

Let $H_{i}:=v_{0} B_{1} v_{1} \ldots v_{k-1} B_{k} v_{k}$, with $v_{0}=v_{k}=r$ when $i=1$, and $g\left(v_{0}\right)<g\left(v_{k}\right)$ when $i \neq 1$. For each 2-connected $B_{j}$, let $u_{j}, w_{j}$ denote the terminals of $B_{j}^{+}$other than $v_{j-1}, v_{j}$, with $f\left(u_{j}\right)<f\left(w_{j}\right)$, and let $T_{v_{j-1}}^{j}, T_{v_{j}}^{j}, T_{u_{j}}^{j}, T_{w_{j}}^{j}$ denote the trees rooted respectively at $v_{j-1}, v_{j}, u_{j}, w_{j}$ in the independent spanning $\left\{v_{j-1}, v_{j}, u_{j}, w_{j}\right\}$-system of $B_{j}^{+}$computed in (4.5). This is the same as in Case 2 of Algorithm Trees. Let $u, v \in D_{i}$ with $g(u)<g(v)$.

If $u, v \in D_{i-1}$, then by the induction hypothesis, $T_{1}[r, u]$ and $T_{2}[r, v]$ are internally disjoint paths in $G_{i-1}$.

If $u \in D_{i}-D_{i-1}$ and $v \in D_{i-1}$, then by the construction in Case 2 of Algorithm Numbering $g$, $g\left(v_{0}\right)<g(u)<g(v)$. By the induction hypothesis, $T_{1}\left[r, v_{0}\right]$ and $T_{2}[r, v]$ are internally disjoint paths in $G_{i-1}$. Since $T_{1}\left[v_{0}, u\right]$ is a path in $H_{i}-v_{k}$ by (1) of Lemma (6.3) when $i=1$, or by (1) of Lemma (6.4) when $i \neq 1, T_{1}[r, v]$ and $T_{2}[r, v]$ are internally disjoint paths in $G_{i}$.

If $u \in D_{i-1}$ and $v \in D_{i}-D_{i-1}$, then by the construction in Case 2 of Algorithm Numbering $g$, $g(u)<g(v)<g\left(v_{k}\right)$. By the induction hypothesis, $T_{1}[r, u]$ and $T_{2}\left[r, v_{k}\right]$ are internally disjoint paths in $G_{i-1}$. Since $T_{2}\left[v_{k}, v\right]$ is a path in $H_{i}-v_{0}$ by (2) of Lemma (6.3) when $i=1$, or by (2) of Lemma (6.4) when $i \neq 1, T_{1}[r, v]$ and $T_{2}[r, v]$ are internally disjoint paths in $G_{i}$.

So we may assume that $u, v \in D_{i}-D_{i-1}$. Let $g^{i}$ denote the function $g$ at the start of iteration $i$ of Algorithm Numbering $g$ (when it examines $H_{i}$ in Case 2). Recall that for each $j=1, \ldots, k$ the algorithm computes a sequence $\sigma_{j}$ as follows. If $B_{j}$ is 2 connected, then $\sigma_{j}$ is a $\left(T_{v_{j-1}}^{j}, T_{v_{j}}^{j}\right)$ ordering of $N_{B_{j}^{+}}\left(\left\{u_{j}, w_{j}\right\}\right)-\left\{v_{j-1}, v_{j}\right\}$. If $B_{j}$ is trivial, then $\sigma_{j}$ is the empty sequence. Moreover, the algorithm extends $g^{i}$ to $\sigma:=\sigma_{1}, v_{1}, \sigma_{2}, \ldots, v_{k-1}, \sigma_{k}$ from $v_{0}$ and set $D_{i}:=D_{i-1} \cup\{\sigma\}$. Thus, $u, v \in\{\sigma\}$. Note that since $g(u)<g(v), u$ precedes $v$ in the sequence $\sigma$.

First, suppose that there exists no $j \in\{1, \ldots, k-1\}$ such that $u, v \in\left\{\sigma_{j}\right\}$. Hence, there is some $j \in\{1, \ldots, k-1\}$ such that either

- $u$ appears in the sequence $\sigma_{1}, v_{1}, \ldots, \sigma_{j}, v_{j}$ and $v$ appears in the sequence $\sigma_{j+1}, v_{j+1}, \ldots, v_{k-1}, \sigma_{k}$, or

- $u$ appears in the sequence $\sigma_{1}, v_{1}, \ldots, \sigma_{j}$ and $v$ appears in the sequence $v_{j}, \sigma_{j+1}, v_{j+1}, \ldots, v_{k-1}, \sigma_{k}$.

By (1) and (2) of Lemma (6.3) when $i=1$ or by (1) and (2) of Lemma (6.4) when $i \neq 1$, $T_{1}\left[v_{0}, u\right]$ and $T_{2}\left[v_{k}, v\right]$ are internally disjoint paths in $H_{i}$, and by the induction hypothesis, $T_{1}\left[r, v_{0}\right]$ and $T_{2}\left[r, v_{k}\right]$ are internally disjoint paths in $G_{i-1}$. Therefore, $T_{1}[r, u]$ and $T_{2}[r, v]$ are internally disjoint paths in $G_{i}$. 
So, we may assume that there exists some $j \in\{1, \ldots, k-1\}$ such that $u, v$ are in the sequence $\sigma_{j}$. Since the sequence $\sigma_{j}$ is $\left(T_{v_{j-1}}^{j}, T_{v_{j}}^{j}\right)$-ordered and $u$ precedes $v$ in $\sigma_{j}, T_{v_{j-1}}^{j}\left[v_{j-1}, u\right]$ and $T_{v_{j}}^{j}\left[v_{j}, v\right]$ are disjoint. By the construction in Algorithm Trees, $T_{1}\left[v_{j-1}, u\right]=T_{v_{j-1}}^{j}\left[v_{j-1}, u\right]$ and $T_{2}\left[v_{j}, v\right]=T_{v_{j}}^{j}\left[v_{j}, v\right]$. By (1) and (2) of Lemma (6.3) when $i=1$, or by (1) and (2) of Lemma (6.4) when $i \neq 1, T_{1}\left[v_{0}, v_{j-1}\right]$ and $T_{2}\left[v_{k}, v_{j}\right]$ are internally disjoint paths in $H_{i}$. Moreover, by the induction hypothesis, $T_{1}\left[r, v_{0}\right]$ and $T_{2}\left[r, v_{k}\right]$ are internally disjoint paths in $G_{i-1}$. Therefore, $T_{1}[r, u]$ and $T_{2}[r, v]$ are internally disjoint paths in $G_{i}$.

Case 3: $H_{i}$ is a down $G_{i-1}$-chain in $G$, but not an elementary $G_{i-1}$-chain in $G$.

Let $H_{i}:=v_{0} B_{1} v_{1} \ldots v_{k-1} B_{k} v_{k}$, with $v_{0}=v_{k}=r$ when $i=t$, and $f\left(v_{0}\right)<f\left(v_{k}\right)$ when $i \neq t$. For each 2-connected $B_{j}$, let $u_{j}, w_{j}$ denote the terminals of $B_{j}^{+}$other than $v_{j-1}, v_{j}$, with $g\left(u_{j}\right)<g\left(w_{j}\right)$, and let $T_{v_{j-1}}^{j}, T_{v_{j}}^{j}, T_{u_{j}}^{j}, T_{w_{j}}^{j}$ denote the trees rooted respectively at $v_{j-1}, v_{j}, u_{j}, w_{j}$ in the independent spanning $\left\{v_{j-1}, v_{j}, u_{j}, w_{j}\right\}$-system of $B_{j}^{+}$computed in (4.5). This is the same as in Case 3 of Algorithm Trees. Let $u, v \in D_{i}$ with $g(u)<g(v)$. Recall that $D_{i}=$ $D_{i-1} \cup N_{B_{1}}\left(v_{0}\right) \cup N_{B_{k}}\left(v_{k}\right)$.

If $u, v \in D_{i-1}$, then by the induction hypothesis, $T_{1}[r, u]$ and $T_{2}[r, v]$ are internally disjoint paths in $G_{i-1} \subset G_{i}$.

If $u \in D_{i}-D_{i-1}$ and $v \in D_{i-1}$, then $u \in N_{B_{1}}\left(v_{0}\right) \cup N_{B_{k}}\left(v_{k}\right)$. By (1) and (3) of Lemma (6.11), there exists $z_{1} \in V\left(G_{i-1}\right)$ such that $g\left(z_{1}\right)<g(u)$ and $V\left(T_{1}\left[z_{1}, u\right]-z_{1}\right) \subseteq I\left(H_{i}\right)$. Since $z_{1}, v \in D_{i-1}$ and $g\left(z_{1}\right)<g(u)<g(v)$, it follows from the induction hypothesis that $T_{1}\left[r, z_{1}\right]$ and $T_{2}[r, v]$ are internally disjoint paths in $G_{i-1}$. Therefore, $T_{1}[r, u]$ and $T_{2}[r, v]$ are internally disjoint paths in $G_{i}$.

If $u \in D_{i-1}$ and $v \in D_{i}-D_{i-1}$, then $v \in N_{B_{1}}\left(v_{0}\right) \cup N_{B_{k}}\left(v_{k}\right)$. By (1) and (3) of Lemma (6.11), there exists $z_{2} \in V\left(G_{i-1}\right)$ such that $g(v)<g\left(z_{2}\right)$ and $V\left(T_{2}\left[z_{2}, v\right]-z_{2}\right) \subseteq I\left(H_{i}\right)$. Since $z_{2}, u \in D_{i-1}$ and $g(u)<g(v)<g\left(z_{2}\right)$, it follows from the induction hypothesis, $T_{1}[r, u]$ and $T_{2}\left[r, z_{2}\right]$ are internally disjoint paths in $G_{i-1}$. Therefore, $T_{1}[r, u]$ and $T_{2}[r, v]$ are internally disjoint paths in $G_{i}$.

So, we need only to prove the case when $u, v \in D_{i}-D_{i-1}$. Let $g^{i}$ denote the function $g$ at the start of iteration $i$ of Algorithm Numbering $g$ (when it examines $H_{i}$ in Case 3 ). Now we consider three cases as in Case 3 of Algorithm Numbering $g$.

Subcase 3.1: $k=1$ (thus, $B_{1}$ is 2-connected).

Since $\left(B_{1}^{+}, v_{0}, u_{1}, v_{1}, w_{1}\right)$ is planar and $G$ is 4-connected, $v_{0}, v_{1} \notin N_{B_{1}}\left(v_{0}\right) \cup N_{B_{1}}\left(v_{1}\right)$. Hence in this case, $D_{i}-D_{i-1}=N_{B_{1}^{+}}\left(\left\{v_{0}, v_{1}\right\}\right)-\left\{u_{1}, w_{1}\right\}=N_{B_{1}}\left(v_{0}\right) \cup N_{B_{1}}\left(v_{1}\right)$. Moreover, Algorithm Numbering $g$ produces a $\left(T_{u_{1}}^{1}, T_{w_{1}}^{1}\right)$-ordering $\sigma$ of $N_{B_{1}^{+}}\left(\left\{v_{0}, v_{1}\right\}\right)-\left\{u_{1}, w_{1}\right\}$ and extends $g^{i}$ to $\sigma$ from $u_{1}$.

Let $u, v \in D_{i}-D_{i-1}$, with $g(u)<g(v)$. Then both $u$ and $v$ are in the sequence $\sigma$, and $u$ precedes $v$ in $\sigma$. Since $\sigma$ is $\left(T_{u_{1}}^{1}, T_{w_{1}}^{1}\right)$-ordered, $T_{u_{1}}^{1}\left[u_{1}, u\right]$ and $T_{w_{1}}^{1}\left[w_{1}, v\right]$ are disjoint. By the construction in Case 3 of Algorithm Trees, $T_{1}\left[u_{1}, u\right]=T_{u_{1}}^{1}\left[u_{1}, u\right]$ and $T_{2}\left[w_{1}, v\right]=T_{w_{1}}^{1}\left[w_{1}, v\right]$. By the induction hypothesis, $T_{1}\left[r, u_{1}\right]$ and $T_{2}\left[r, w_{1}\right]$ are internally disjoint paths in $G_{i-1}$. Therefore, $T_{1}[r, u]$ and $T_{2}[r, v]$ are internally disjoint paths in $G_{i}$.

Subcase 3.2: $k=2$, and $B_{1}$ or $B_{2}$ is trivial.

By symmetry we assume that $B_{2}$ is trivial (the arguments are analogous if $B_{1}$ is trivial). Note that $B_{1}$ is 2 -connected because $H_{i}$ is not an elementary $G_{i-1}$-chain in $G$. Thus, $D_{i}-D_{i-1}=$ $N_{B_{1}}\left(v_{0}\right) \cup\left\{v_{1}\right\}$.

- If $v_{1}$ has no neighbor in $V\left(G_{i-1}\right)$, then Algorithm Numbering $g$ chooses neighbors $q_{1}, q_{2}, q_{3}$ of $v_{1}$ in $B_{1}$ such that $T_{u_{1}}^{1}\left[u_{1}, q_{1}\right], T_{v_{0}}^{1}\left[v_{0}, q_{2}\right]$ and $T_{w_{1}}^{1}\left[w_{1}, q_{3}\right]$ are disjoint, and then computes a $\left(T_{u_{1}}^{1}+\left\{v_{1}, v_{1} q_{1}\right\}, T_{w_{1}}^{1}+\left\{v_{1}, v_{1} q_{3}\right\}\right)$ )-ordering $\sigma$ of $N_{B_{1}}\left(v_{0}\right) \cup\left\{v_{1}\right\}$ in $B_{1}^{+} \cup B_{2}$ (recall that $\left(B_{1}^{+} \cup B_{2}, v_{0}, u_{1}, v_{2}, w_{1}\right)$ is planar). Then Algorithm Numbering $g$ extends $g^{i}$ to $\sigma$ from $u_{1}$.

Let $u, v \in D_{i}-D_{i-1}$, with $g(u)<g(v)$. Then both $u$ and $v$ are in the sequence $\sigma$, and $u$ precedes $v$ in $\sigma$. 
Let us consider first the case when $u \neq v_{1}$ and $v \neq v_{1}$. Thus, $u, v \in N_{B_{1}}\left(v_{0}\right)$. Since $\sigma$ is $\left(T_{u_{1}}^{1}+\left\{v_{1}, v_{1} q_{1}\right\}, T_{w_{1}}^{1}+\left\{v_{1}, v_{1} q_{3}\right\}\right)$-ordered and $u$ precedes $v$ in $\sigma, T_{u_{1}}^{1}\left[u_{1}, u\right]$ and $T_{w_{1}}^{1}\left[w_{1}, v\right]$ are disjoint. By construction (Case 3 of Algorithm Trees), $T_{1}\left[u_{1}, u\right]=T_{u_{1}}^{1}\left[u_{1}, u\right]$ and $T_{2}\left[w_{1}, v\right]=T_{w_{1}}^{1}\left[w_{1}, v\right]$. By the induction hypothesis, $T_{1}\left[r, u_{1}\right]$ and $T_{2}\left[r, w_{1}\right]$ are internally disjoint paths in $G_{i-1}$. Therefore, $T_{1}[r, u]$ and $T_{2}[r, v]$ are internally disjoint paths in $G_{i}$.

Now suppose that $u=v_{1}$. Since $\sigma$ is $\left(T_{u_{1}}^{1}+\left\{v_{1}, v_{1} q_{1}\right\}, T_{w_{1}}^{1}+\left\{v_{1}, v_{1} q_{3}\right\}\right)$-ordered and $u$ precedes $v$ in $\sigma, T_{u_{1}}^{1}\left[u_{1}, q_{1}\right]+\left\{v_{1}, v_{1} q_{1}\right\}$ and $T_{w_{1}}^{1}\left[w_{1}, v\right]$ are disjoint. By construction (Case 3 of Algorithm Trees), $T_{1}\left[u_{1}, v_{1}\right]=T_{u_{1}}^{1}\left[u_{1}, q_{1}\right]+\left\{v_{1}, v_{1} q_{1}\right\}$ and $T_{2}\left[w_{1}, v\right]=T_{w_{1}}^{1}\left[w_{1}, v\right]$. By the induction hypothesis, $T_{1}\left[r, u_{1}\right]$ and $T_{2}\left[r, w_{1}\right]$ are internally disjoint paths in $G_{i-1}$. Therefore, $T_{1}[r, u]$ and $T_{2}[r, v]$ are internally disjoint paths in $G_{i}$.

So assume $v=v_{1}$. Since $\sigma$ is $\left(T_{u_{1}}^{1}+\left\{v_{1}, v_{1} q_{1}\right\}, T_{w_{1}}^{1}+\left\{v_{1}, v_{1} q_{3}\right\}\right)$-ordered and $u$ precedes $v$ in $\sigma, T_{u_{1}}^{1}\left[u_{1}, u\right]$ and $T_{w_{1}}^{1}\left[w_{1}, q_{3}\right]+\left\{v_{1}, v_{1} q_{3}\right\}$ are disjoint. By construction (Case 3 of Algorithm Trees), $T_{1}\left[u_{1}, u\right]=T_{u_{1}}^{1}\left[u_{1}, u\right]$ and $T_{2}\left[w_{1}, v_{1}\right]=T_{w_{1}}^{1}\left[w_{1}, q_{3}\right]+\left\{v_{1}, v_{1} q_{3}\right\}$. By the induction hypothesis, $T_{1}\left[r, u_{1}\right]$ and $T_{2}\left[r, w_{1}\right]$ are internally disjoint paths in $G_{i-1}$. Therefore, $T_{1}[r, u]$ and $T_{2}[r, v]$ are internally disjoint paths in $G_{i}$.

- If $v_{1}$ has a neighbor in $V\left(G_{i-1}\right)$, then Algorithm Numbering $g$ chooses a vertex $x \in$ $\left(N_{G}\left(v_{1}\right) \cap V\left(G_{i-1}\right)\right)$ with $g^{i}(x)$ minimum, and computes a $\left(T_{u_{1}}^{1}, T_{w_{1}}^{1}\right)$-ordering $\sigma$ of $N_{B_{1}^{+}}\left(v_{0}\right)-\left\{u_{1}, w_{1}\right\}$.

Let $u, v \in D_{i}-D_{i-1}$, with $g(u)<g(v)$.

Let us consider first the case when $u \neq v_{1}$ and $v \neq v_{1}$. Then both $u$ and $v$ are in $\sigma$, and $u$ precedes $v$ in $\sigma$. Since $\sigma$ is $\left(T_{u_{1}}^{1}, T_{w_{1}}^{1}\right)$-ordered and $u$ precedes $v$ in $\sigma, T_{u_{1}}^{1}\left[u_{1}, u\right]$ and $T_{w_{1}}^{1}\left[w_{1}, v\right]$ are disjoint. By construction (Case 3 of Algorithm Trees), $T_{1}\left[u_{1}, u\right]=T_{u_{1}}^{1}\left[u_{1}, u\right]$ and $T_{2}\left[w_{1}, v\right]=T_{w_{1}}^{1}\left[w_{1}, v\right]$. By the induction hypothesis, $T_{1}\left[r, u_{1}\right]$ and $T_{2}\left[r, w_{1}\right]$ are internally disjoint paths in $G_{i-1}$. Therefore, $T_{1}[r, u]$ and $T_{2}[r, v]$ are internally disjoint paths in $G_{i}$.

Now suppose that $u=v_{1}$. Thus, $v$ is in the sequence $\sigma$. Recall how Algorithm Numbering $g$ extends $g^{i}$ in Subcase 3.2 of Algorithm Numbering $g$.

If $g(x)>g\left(u_{1}\right)$, then $g^{i}(x)>g^{i}\left(u_{1}\right)$ and Algorithm Numbering $g$ extends $g^{i}$ to $\sigma, v_{1}$ from $u_{1}$. But then $g(v)<g\left(v_{1}\right)=g(u)$, contradicting the assumption that $g(u)<g(v)$.

If $g(x) \leq g\left(u_{1}\right)$, then $g^{i}(x) \leq g^{i}\left(u_{1}\right)$ and Algorithm Numbering $g$ extends $g^{i}$ to $v_{1}, \sigma$ from $x$. By construction (Subcase 3.2 of Algorithm Trees with $j=1$ ), $x v_{1} \in E\left(T_{1}\right)$ and $T_{2}\left[w_{1}, v\right]=T_{w_{1}}^{1}\left[w_{1}, v\right]$. Since $g(x)<g\left(w_{1}\right)$, by the induction hypothesis, $T_{1}[r, x]$ and $T_{2}\left[r, w_{1}\right]$ are internally disjoint paths in $G_{i-1}$. Therefore, $T_{1}\left[r, v_{1}\right]$ and $T_{2}[r, v]$ are internally disjoint paths in $G_{i}$.

The case $v=v_{1}$ can be treated analogously $\left(g(x) \leq g\left(u_{1}\right)\right.$ cannot occur).

Subcase 3.3: $k \geq 3$, or $k=2$ and both $B_{1}, B_{2}$ are 2-connected.

In this case, $D_{i}-D_{i-1}=N_{B_{1}}\left(v_{0}\right) \cup N_{B_{k}}\left(v_{k}\right)$. Let $u, v \in D_{i}-D_{i-1}$ with $g(u)<g(v)$.

Let us consider first the case when $u, v \in N_{B_{1}}\left(v_{0}\right)$. Thus, $B_{1}$ is 2-connected, and Algorithm Numbering $g$ (Subcase 3.3) computes a $\left(T_{u_{1}}^{1}, T_{w_{1}}^{1}\right)$-ordering $\sigma$ of $N_{B_{1}^{+}}\left(v_{0}\right)-\left\{u_{1}, w_{1}\right\}=N_{B_{1}}\left(v_{0}\right)$ and extends $g^{i}$ to $\sigma$ from $u_{1}$. Thus, $g\left(u_{1}\right)<g(u)<g(v)$. Since $\sigma$ is $\left(T_{u_{1}}^{1}, T_{w_{1}}^{1}\right)$-ordered and $u$ precedes $v$ in $\sigma, T_{u_{1}}^{1}\left[u_{1}, u\right]$ and $T_{w_{1}}^{1}\left[w_{1}, v\right]$ are disjoint. By construction (Case 3 of Algorithm trees), $T_{1}\left[u_{1}, u\right]=T_{u_{1}}^{1}\left[u_{1}, u\right]$ and $T_{2}\left[w_{1}, v\right]=T_{w_{1}}^{1}\left[w_{1}, v\right]$. Since $g\left(u_{1}\right)<g\left(w_{1}\right)$ and by the induction hypothesis, $T_{1}\left[r, u_{1}\right]$ and $T_{2}\left[r, w_{1}\right]$ are internally disjoint paths in $G_{i-1}$. Therefore, $T_{1}[r, u]$ and $T_{2}[r, v]$ are internally disjoint paths in $G_{i}$.

Suppose, now, that $u, v \in N_{B_{k}}\left(v_{k}\right)$. Then $B_{k}$ is 2-connected, and Algorithm Numbering $g$ (Subcase 3.3) computes a $\left(T_{u_{k}}^{k}, T_{w_{k}}^{k}\right)$-ordering of $N_{B_{k}^{+}}\left(v_{k}\right)-\left\{u_{k}, w_{k}\right\}=N_{B_{k}}\left(v_{k}\right)$ and extends $g^{i}$ to $\sigma$ from $u_{k}$. Thus, $g\left(u_{k}\right)<g(u)<g(v)$. Since $\sigma$ is $\left(T_{u_{k}}^{k}, T_{w_{k}}^{k}\right)$-ordered and $u$ precedes $v$ in $\sigma, T_{u_{k}}^{k}\left[u_{k}, u\right]$ and $T_{w_{k}}^{k}\left[w_{k}, v\right]$ are disjoint. By construction (Case 3 of Algorithm Trees), 
$T_{1}\left[u_{k}, u\right]=T_{u_{k}}^{k}\left[u_{k}, u\right]$ and $T_{2}\left[w_{k}, v\right]=T_{w_{k}}^{k}\left[w_{k}, v\right]$. Since $g\left(u_{k}\right)<g\left(w_{k}\right)$ and by the induction hypothesis, $T_{1}\left[r, u_{k}\right]$ and $T_{2}\left[r, w_{k}\right]$ are internally disjoint paths in $G_{i-1}$. Therefore, $T_{1}[r, u]$ and $T_{2}[r, v]$ are internally disjoint paths in $G_{i}$.

So we may assume that $u \in N_{B_{1}}\left(v_{0}\right)$ and $v \in N_{B_{k}}\left(v_{k}\right)$, or $u \in N_{B_{k}}\left(v_{k}\right)$ and $v \in N_{B_{1}}\left(v_{0}\right)$. By symmetry, assume that $u \in N_{B_{1}}\left(v_{0}\right)$ and $v \in N_{B_{k}}\left(v_{k}\right)$. We will prove that there exist vertices $z_{1}, z_{2} \in V\left(G_{i-1}\right)$ with $g\left(z_{1}\right)<g\left(z_{2}\right)$ such that $T_{1}\left[z_{1}, u\right]$ and $T_{2}\left[z_{2}, v\right]$ are internally disjoint paths in $G, V\left(T_{1}\left[z_{1}, u\right]-z_{1}\right) \subseteq I\left(H_{i}\right)$, and $V\left(T_{2}\left[z_{2}, v\right]-z_{2}\right) \subseteq I\left(H_{i}\right)$.

Consider the following cases for $u$ and $B_{1}$.

- $B_{1}$ is 2-connected. Then, by construction in Algorithm Trees, $T_{1}\left[u_{1}, u\right]=T_{u_{1}}^{1}\left[u_{1}, u\right]$ and let $z_{1}:=u_{1}$.

- $B_{1}$ is trivial. Thus, $u=v_{1}$. If $B_{2}$ is trivial, then by construction in Subcase 3.1 of Algorithm Trees (with $j=1$ ), there exists a neighbor $p_{1}$ of $v_{1}$ in $V\left(G_{i-1}\right)$ such that $g\left(p_{1}\right)$ is minimum and $p_{1} v_{1} \in E\left(T_{1}\right)$. In this case, let $z_{1}:=p_{1}$.

So assume that $B_{2}$ is 2-connected.

- If $v_{1}$ has no neighbor in $V\left(G_{i-1}\right)$, then by construction in Subcase 3.3 (with $j=1$ ) of Algorithm Trees, there exists a neighbor $p_{1}$ of $v_{1}$ in $B_{2}$ such that $T_{1}\left[u_{2}, v_{1}\right]=$ $T_{u_{2}}^{2}\left[u_{2}, p_{1}\right]+\left\{v_{1}, v_{1} p_{1}\right\}$. In this case, let $z_{1}:=u_{2}$.

- If $v_{1}$ has a neighbor in $V\left(G_{i-1}\right)$, then Algorithm Trees in Subcase 3.3 (with $j=$ 1) chooses $x \in N_{G}\left(v_{1}\right) \cap V\left(G_{i-1}\right)$ with $g(x)$ minimum. If $g(x)>g\left(u_{2}\right)$, then by construction there exists a neighbor $p_{1}$ of $v_{1}$ in $B_{2}$ such that $T_{1}\left[u_{2}, v_{1}\right]=T_{u_{2}}^{2}\left[u_{2}, p_{1}\right]+$ $\left\{v_{1}, v_{1} p_{1}\right\}$. In this case, let $z_{1}:=u_{2}$. If $g(x) \leq g\left(u_{2}\right)$, then $x v_{1} \in E\left(T_{1}\right)$. In this case, let $z_{1}:=x$.

Consider the analogous cases for $v$ and $B_{k}$.

- $B_{k}$ is 2-connected. Then by construction in Algorithm Trees, $T_{2}\left[w_{k}, v\right]=T_{w_{k}}^{k}\left[w_{k}, v\right]$ and let $z_{2}:=w_{k}$.

- $B_{k}$ is trivial. Thus, $v=v_{k-1}$. If $B_{k-1}$ is trivial, then by construction in Subcase 3.1 of Algorithm Trees (with $j=k-1$ ), there exists a neighbor $p_{2}$ of $v_{k-1}$ in $V\left(G_{i-1}\right)$ such that $g\left(p_{2}\right)$ is not minimum and $p_{2} v_{k-1} \in E\left(T_{2}\right)$. In this case, let $z_{2}:=p_{2}$.

So assume that $B_{k-1}$ is 2-connected.

- If $v_{k-1}$ has no neighbor in $V\left(G_{i-1}\right)$, then by construction in Subcase 3.2 (with $j=$ $k-1)$ of Algorithm Trees, there exists a neighbor $p_{2}$ of $v_{k-1}$ in $B_{k-1}$ such that $T_{2}\left[w_{k-1}, v_{k-1}\right]=T_{w_{k-1}}^{k-1}\left[w_{k-1}, p_{2}\right]+\left\{v_{k-1}, v_{k-1} p_{2}\right\}$. In this case, let $z_{2}:=w_{k-1}$.

- If $v_{k-1}$ has a neighbor in $V\left(G_{i-1}\right)$, then Algorithm Trees in Subcase 3.2 chooses $x \in N_{G}\left(v_{k-1}\right) \cap V\left(G_{i-1}\right)$ with $g(x)$ minimum. If $g(x)>g\left(u_{k-1}\right)$, then $x v_{k-1} \in E\left(T_{2}\right)$. In this case, let $z_{2}:=x$. If $g(x) \leq g\left(u_{k-1}\right)$, then by construction there exists a neighbor $p_{2}$ of $v_{k-1}$ in $B_{k-1}$ such that $T_{2}\left[w_{k-1}, v_{k-1}\right]=T_{w_{k-1}}^{k-1}\left[w_{k-1}, p_{2}\right]+\left\{v_{k-1}, v_{k-1} p_{2}\right\}$. In this case, let $z_{2}:=w_{k-1}$ (this is the same as in the last paragraph).

So $T_{1}\left[z_{1}, u\right]$ either is contained in $B_{1}^{+}$, or is contained in $B_{2}^{+}$, or is induced by a single edge. Hence $g\left(z_{1}\right)<g(u)$. Similarly, $T_{2}\left[z_{2}, v\right]$ either is contained in $B_{k}^{+}$, or is contained in $B_{k-1}^{+}$, or is induced by a single edge. So $g(v)<g\left(z_{2}\right)$. Since $g(u)<g(v), g\left(z_{1}\right)<g\left(z_{2}\right)$.

Note that if $k=3, B_{2}$ is 2 -connected and both paths $T_{1}\left[z_{1}, u\right]$ and $T_{2}\left[z_{2}, v\right]$ are contained in $B_{2}^{+}$, then $u=v_{1}, v=v_{2}=v_{k-1}, T_{1}\left[u_{2}, u\right]=T_{u_{2}}^{2}\left[u_{2}, p_{1}\right]+\left\{v_{1}, v_{1} p_{1}\right\}$ for some neighbor $p_{1}$ of $v_{1}$ in $B_{2}$, and $T_{2}\left[w_{2}, v\right]=T_{w_{2}}^{2}\left[w_{2}, p_{2}\right]+\left\{v_{2}, v_{2} p_{2}\right\}$ for some neighbor $p_{2}$ of $v_{2}$ in $B_{2}$. In this case, since $u, v$ are $\left(T_{u_{2}}^{2}, T_{w_{2}}^{2}\right)$-ordered, $T_{1}\left[u_{2}, u\right]$ and $T_{2}\left[w_{2}, v\right]$ are disjoint.

Therefore, since $k \geq 3$, it is not hard to see that $T_{1}\left[z_{1}, u\right]$ and $T_{2}\left[z_{2}, v\right]$ are disjoint paths in $G, V\left(T_{1}\left[z_{1}, u\right]-z_{1}\right) \subset I\left(H_{i}\right)$, and $V\left(T_{2}\left[z_{2}, v\right]-z_{2}\right) \subset I\left(H_{i}\right)$. 
Since $g\left(z_{1}\right)<g\left(z_{2}\right)$, by the induction hypothesis, $T_{1}\left[r, z_{1}\right]$ and $T_{2}\left[r, z_{2}\right]$ are internally disjoint paths in $G_{i-1}$. Therefore, $T_{1}[r, u]$ and $T_{2}[r, v]$ are internally disjoint paths in $G_{i}$.

Case 4: $H_{i}$ is a triangle $G_{i-1}$-chain in $G$.

By Algorithm Numbering $g, D_{i}-D_{i-1}=\left\{v_{1}, v_{2}, v_{3}\right\}$ and $g\left(v_{1}\right)<g\left(v_{2}\right)<g\left(v_{3}\right)$. Thus, it suffices to show that the following pairs are internally disjoint: $T_{1}\left[r, v_{1}\right]$ and $T_{2}\left[r, v_{2}\right], T_{1}\left[r, v_{2}\right]$ and $T_{2}\left[r, v_{3}\right]$, and $T_{1}\left[r, v_{1}\right]$ and $T_{2}\left[r, v_{3}\right]$. This can be done by inspecting Case 4 of Algorithm Trees.

Recall that Algorithm Numbering $f$ with input $\mathcal{C}^{\prime}:=H_{t}, \ldots, H_{1}$ computes a numbering $f$ and sets $D_{t+1}^{\prime}, D_{t}^{\prime}, D_{t-1}^{\prime}, \ldots, D_{2}^{\prime}$. The next lemma can be proved, analogously to Lemma (6.12). We only give some detail for Case 4 , as $f$ and $g$ are not symmetric in that case.

(6.13) Lemma. Let $i \in\{1, \ldots, t\}$. Then for any $u, v \in D_{i}^{\prime}$ with $f(u)<f(v), T_{3}[r, u]$ and $T_{4}[r, v]$ are internally disjoint paths in $\bar{G}_{i}$.

Proof. We use the notation in the proof of Lemma (6.12) and assume $H_{i}$ is a triangle $G_{i-1^{-}}$ chain in $G$. By inspecting Case 4 of Algorithm Numbering $f$ and Algorithm Trees, we have the following.

- If $f\left(y_{1}\right)<f\left(y_{2}\right)$ and $f\left(y_{1}\right)<f\left(y_{3}\right)$, then $f\left(v_{1}\right)<f\left(v_{2}\right)<f\left(v_{3}\right)$. So we can show that $T_{3}\left[r, v_{1}\right]$ and $T_{4}\left[r, v_{2}\right]$ are internally disjoint, $T_{3}\left[r, v_{1}\right]$ and $T_{4}\left[r, v_{3}\right]$ are internally disjoint, and $T_{3}\left[r, v_{2}\right]$ and $T_{4}\left[r, v_{3}\right]$ are internally disjoint.

- If $f\left(y_{2}\right)<f\left(y_{1}\right)$ and $f\left(y_{2}\right)<f\left(y_{3}\right)$, then $f\left(v_{2}\right)<f\left(v_{1}\right)<f\left(v_{3}\right)$. So we can show that $T_{3}\left[r, v_{2}\right]$ and $T_{4}\left[r, v_{1}\right]$ are internally disjoint, $T_{3}\left[r, v_{2}\right]$ and $T_{4}\left[r, v_{3}\right]$ are internally disjoint, and $T_{3}\left[r, v_{1}\right]$ and $T_{4}\left[r, v_{3}\right]$ are internally disjoint.

- If $f\left(y_{3}\right)<f\left(y_{1}\right)<f\left(y_{2}\right)$, then $f\left(v_{3}\right)<f\left(v_{1}\right)<f\left(v_{2}\right)$. So we can show that $T_{3}\left[r, v_{3}\right]$ and $T_{4}\left[r, v_{1}\right]$ are internally disjoint, $T_{3}\left[r, v_{3}\right]$ and $T_{4}\left[r, v_{2}\right]$ are internally disjoint, and $T_{3}\left[r, v_{1}\right]$ and $T_{4}\left[r, v_{2}\right]$ are internally disjoint.

- If $f\left(y_{3}\right)<f\left(y_{2}\right)<f\left(y_{1}\right)$, then $f\left(v_{3}\right)<f\left(v_{2}\right)<f\left(v_{1}\right)$. So we can show that $T_{3}\left[r, v_{3}\right]$ and $T_{4}\left[r, v_{2}\right]$ are internally disjoint, $T_{3}\left[r, v_{3}\right]$ and $T_{4}\left[r, v_{1}\right]$ are internally disjoint, and $T_{3}\left[r, v_{2}\right]$ and $T_{4}\left[r, v_{1}\right]$ are internally disjoint.

(6.14) Theorem. Given a 4-connected graph $G, r \in V(G)$, and a non-separating chain decomposition $\mathcal{C}$ of $G$ rooted at $r$, Algorithm Trees computes four independent spanning trees rooted at $r$.

Proof. By Corollary (6.10), $T_{1}, T_{2}, T_{3}, T_{4}$ are spanning trees of $G$. Let us prove that they are independent with $r$ as root. Let $v \in V(G)-\{r\}$. Suppose that $v$ is an internal vertex of a good chain $H_{i}$ in the decomposition $\mathcal{C}$. By Lemma (6.11) there exist $z_{1}, z_{2}, z_{3}, z_{4} \in V(G)$ such that

(i) $z_{1}, z_{2} \in V\left(G_{i-1}\right)$, and either $g\left(z_{1}\right)<g\left(z_{2}\right)$ or $z_{1}=z_{2}=r$,

(ii) $z_{3}, z_{4} \in V\left(\bar{G}_{i}\right)$, and either $f\left(z_{3}\right)<f\left(z_{4}\right)$ or $z_{3}=z_{4}=r$, and

(iii) $T_{i}\left[z_{i}, v\right], i=1,2,3,4$ are internally disjoint paths and $V\left(T_{i}\left[z_{i}, v\right]-z_{i}\right) \subseteq I\left(H_{i}\right)$.

By Lemma (6.12), if $g\left(z_{1}\right)<g\left(z_{2}\right)$, then $T_{1}\left[r, z_{1}\right]$ and $T_{2}\left[r, z_{2}\right]$ are internally disjoint paths in $G_{i-1}$. Obviously, the same holds if $z_{1}=z_{2}=r$. Similarly, by Lemma (6.13), if $f\left(z_{3}\right)<f\left(z_{4}\right)$, then $T_{3}\left[r, z_{3}\right]$ and $T_{4}\left[r, z_{4}\right]$ are internally disjoint paths in $\bar{G}_{i}$, and, the same holds if $z_{3}=z_{4}=r$. Therefore, $T_{1}[r, v], T_{2}[r, v], T_{3}[r, v]$ and $T_{4}[r, v]$ are internally disjoint. Hence, $T_{1}, T_{2}, T_{3}$ and $T_{4}$ are independent spanning trees of $G$ rooted at $r$. 
(6.15) Lemma. Algorithm Trees runs in $O\left(|V(G)|^{3}\right)$ time.

Proof. By Theorems (4.6) and (4.7), given $\mathcal{C}$ we can compute numberings $g$ and $f$ in $O\left(|V(G)|^{3}\right)$ time. By Theorem (3.2) we can compute independent spanning systems for all planar sections in $\mathcal{C}$ in $O(|V(G)|+|E(G)|)$ time.

We will show that at each iteration the time spent by Algorithm Trees is $O\left(|V(G)|^{2}\right)$ time. Since the number of iterations is at most $|V(G)|$, this implies the result.

Suppose we are at iteration $i$ of Algorithm Trees.

One can see easily that if Case 1 or Case 4 occurs, then Algorithm Trees uses constant time. Thus, we may assume that Case 2 or Case 3 occurs.

Suppose that Case 2 occurs. The initial updating of $T_{1}, T_{2}, T_{3}, T_{4}$ (before the Subcases 2.1-2.4 are dealt with) can be done in $O(|V(G)|)$ time. Then for each $j \in\{1, \ldots, k\}$ the algorithm inserts $v_{j}$ into the subgraphs $T_{1}, T_{2}, T_{3}, T_{4}$ according to the Subcases 2.1-2.4. One can see that Subcase 2.1 can be executed in $O(1)$ time. In the other cases, the algorithm has to solve one of the following problems (at most twice).

(1) Given a planar graph $\left(B, v^{\prime}, u, v, w\right)$ and an independent spanning $\left\{v^{\prime}, u, v, w\right\}$-system $\left\{T_{v^{\prime}}, T_{u}, T_{v}, T_{w}\right\}$ of $B$ (with $T_{v^{\prime}}, T_{u}, T_{v}, T_{w}$ rooted respectively at $v^{\prime}, u, v, w$ ), find three neighbors $p_{1}, p_{2}, p_{3}$ of $v$ in $B$ such that $T_{v^{\prime}}\left[v^{\prime}, p_{1}\right], T_{u}\left[u, p_{2}\right]$ and $T_{w}\left[w, p_{3}\right]$ are disjoint.

(2) Given a planar graph $\left(B, v^{\prime}, u, v, w\right)$ and an independent spanning $\left\{v^{\prime}, u, v, w\right\}$-system $\left\{T_{v^{\prime}}, T_{u}, T_{v}, T_{w}\right\}$ of $B$ (with $T_{v^{\prime}}, T_{u}, T_{v}, T_{w}$ rooted respectively at $v^{\prime}, u, v, w$ ), find two neighbors $p_{1}, p_{2}$ of $v$ in $B$ such that $T_{v^{\prime}}\left[v^{\prime}, p_{1}\right]$ and $T_{u}\left[u, p_{2}\right]$ are disjoint.

By Lemmas (3.6) and (3.7), both problems can be solved in $O(|V(B)|)$ time. Thus, it is not hard so see that the time spent by Algorithm Trees in Case 2 is $O\left(|V(G)|^{2}\right)$.

Case 3 is analogous to Case 2, and by an argument similar to the last paragraph, one can show that Algorithm Trees uses $O\left(|V(G)|^{2}\right)$ time in this case as well.

Now we are almost ready to prove Theorem (1.1), except that if we apply Theorem (2.8) directly to a 4-connected graph $G$ to find a non-separating chain decomposition of $G$, we spend $O\left(|V(G)|^{2}|E(G)|\right)$ time. We can obtain an $O\left(|V(G)|^{3}\right)$ algorithm by using the following result of Ibaraki and Nagamochi [9].

(6.16) Theorem. Let $G$ be a $k$-connected graph for some integer $k \geq 1$. Then one can find in $O(|V(G)|+|E(G)|)$ time a spanning $k$-connected subgraph of $G$ with $O(|V(G)|)$ edges.

Proof of Theorem (1.1). Let $G$ be a 4-connected graph, and let $r \in V(G)$. Apply Theorem (6.16) to $G$ and let $G^{\prime}$ be the resulting spanning 4-connected subgraph of $G$.

Applying Theorem (2.8) to $G^{\prime}$, we can find a non-separating chain decomposition $\mathcal{C}$ of $G^{\prime}$ in $O\left(\left|V\left(G^{\prime}\right)\right|^{3}\right)$ time (and hence, in $O\left(|V(G)|^{3}\right)$ time).

Finally, apply Theorem (6.14) to $G, \mathcal{C}$ and find four independent spanning trees $T_{1}, T_{2}, T_{3}, T_{4}$ of $G^{\prime}$ rooted at $r$. By (6.15), this is done in $O\left(\left|V\left(G^{\prime}\right)\right|^{3}\right)$ time, and hence in $O\left(|V(G)|^{3}\right)$ time. Clearly, $T_{1}, T_{2}, T_{3}, T_{4}$ are independent spanning trees of $G$ rooted at $r$.

\section{References}

[1] S. Curran, Independent Trees in 4-Connected Graphs, Ph.D Thesis, Georgia Institute of Technology, 2003.

[2] S. Curran, O. Lee and X. Yu. Decomposing 4-connected graphs. Submitted, 2003. (Also see http://www.math.gatech.edu/ yu/Papers/tree.pdf)

[3] J. Cheriyan and S.N. Maheshwari. Finding nonseparating induced cycles and independent spanning trees in 3-connected graphs. J. Algorithms 9 (1988), 507-537. 
[4] T.H. Cormen, C.E. Leiserson, R.L. Rivest and S. Clifford. Introduction to algorithms. Second edition. McGraw-Hill Book Co., Boston, MA, 2001.

[5] D. Dolev, J.Y Halpern, B. Simons and R. Strong. A new look at fault tolerant network routing. Proc. 16th Annual ACM Symp. on Theory of Comput. (1984), 526-535.

[6] J.E. Hopcroft and R.E. Tarjan. Efficient planarity testing. J. Assoc. Comp. Mach. 21 (1974), 549-568.

[7] W.-L. Hsu and W.-K. Shih. A new planarity test. Theoretical Comp. Sci. 223 (1999), $179-191$.

[8] A. Huck. Independent trees in graphs. Graphs and Combinatorics 10 (1994), 29-45.

[9] T. Ibaraki and H. Nagamochi. A linear-time algorithm for finding a sparse $k$-connected spanning subgraph of a $k$-connected graph. Algorithmica 7 (1992), 583-596.

[10] A. Itai and M. Rodeh. The multi-tree approach to reliability in distributed networks. Proc. 25th Annual IEEE Symp. Found. Comp. Sci. (1984), 137-147.

[11] A. Itai and A. Zehavi. Three tree-paths. J. Graph Theory 13 (1989), 175-188.

[12] K. Miura, S. Nakano, T. Nishizeki and D. Takahashi. A linear-time algorithm to find four independent spanning trees in four connected planar graphs. Int. J. Found. Comp. Sci. 10 (1999), 195-210. 